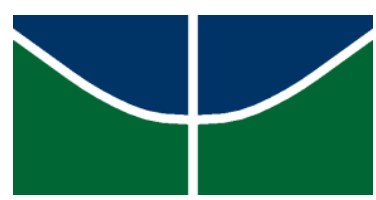

Universidade de Brasília

Instituto de Psicologia

Programa de Pós-Graduação em Psicologia Social, do Trabalho e das Organizações

\title{
AVALIAÇÃO DA IGUALDADE, EQUIDADE E EFICÁCIA NO SISTEMA EDUCACIONAL BRASILEIRO
}

\author{
Camila Akemi Karino \\ Orientador: Prof. PhD. Jacob Arie Laros
}

Brasília, junho de 2016. 


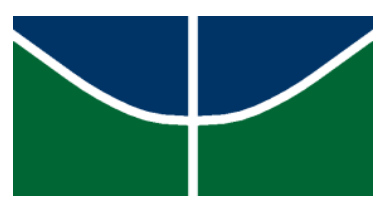

Universidade de Brasília

Instituto de Psicologia

Programa de Pós-Graduação em Psicologia Social, do Trabalho e das Organizações

\section{AVALIAÇÃO DA IGUALDADE, EQUIDADE E EFICÁCIA NO SISTEMA EDUCACIONAL BRASILEIRO}

Camila Akemi Karino

Tese elaborada sob orientação do Prof. PhD. Jacob Arie Laros, apresentada ao Programa de Pós-Graduação em Psicologia Social, do Trabalho e das Organizações da Universidade de Brasília, como requisito parcial à obtenção do título de Doutor em Psicologia Social, do Trabalho e das Organizações.

Brasília, junho de 2016. 
Universidade de Brasília

Instituto de Psicologia

Programa de Pós-Graduação em Psicologia Social, do Trabalho e das Organizações

Tese aprovada pela seguinte banca examinadora:

Prof. PhD. Jacob Arie Laros (Presidente)

Universidade de Brasília - UnB

Prof. PhD. José Francisco Soares (Membro)

Universidade Federal de Minas Gerais - UFMG

Prof. PhD. Joaquim José Soares Neto (Membro)

Universidade de Brasília - UnB

Profa. Dra. Cristiane Faiad (Membro)

Universidade de Brasília - UnB

Prof. Dr. Fabio Iglesias (Membro)

Universidade de Brasília - UnB

Brasília, junho de 2016 


\section{Sumário}

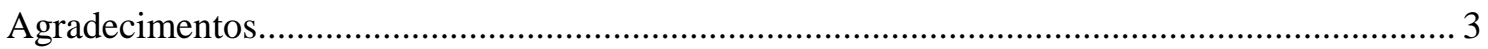

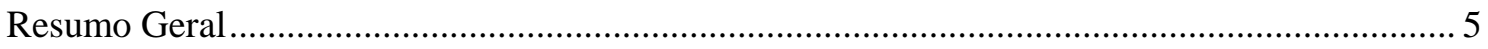

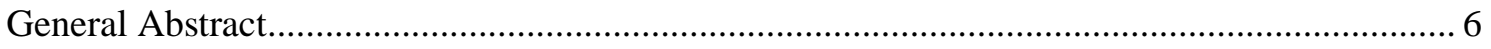

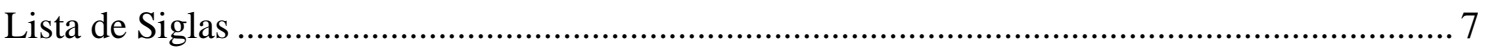

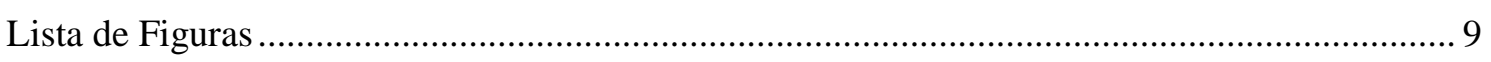

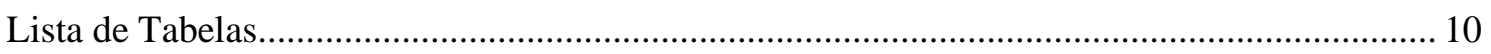

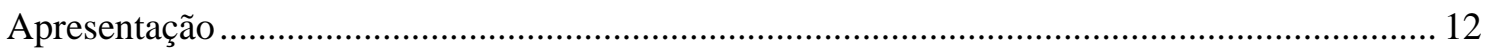

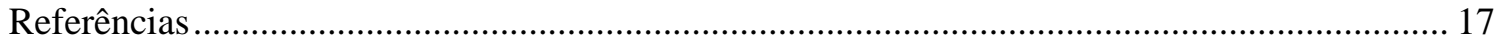

\section{Manuscrito 1}

ESTUDOS BRASILEIROS SOBRE EFICÁCIA ESCOLAR:_UMA REVISÃO DE LITERATURA

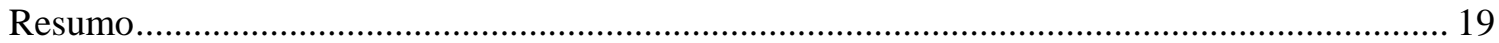

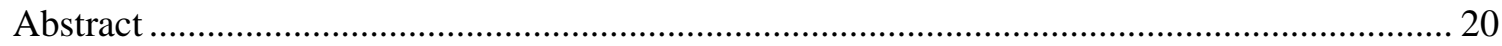

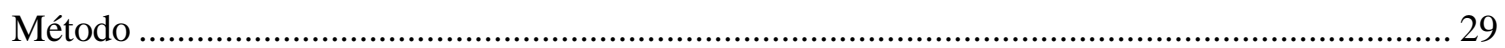

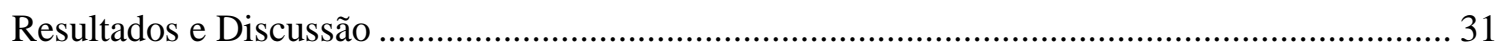

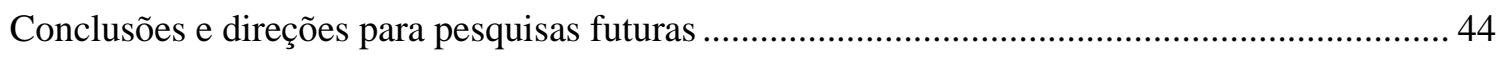

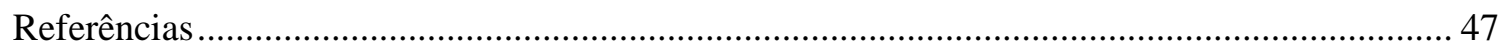

\section{Manuscrito 2}

PILLARS OF GOOD EDUCATION: ANALYZING_THE BRAZILIAN SCHOOL SYSTEM....53

Abstract




\section{Manuscrito 3}

AVALIAÇÃO DA IGUALDADE E EQUIDADE_DO SISTEMA EDUCACIONAL PÚBLICO BRASILEIRO.

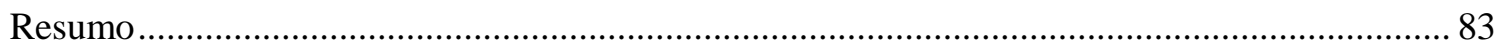

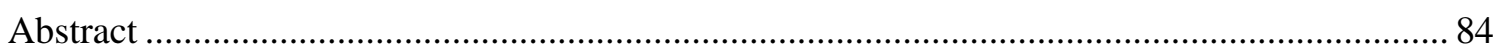

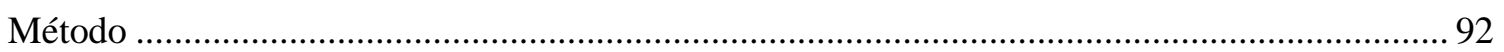

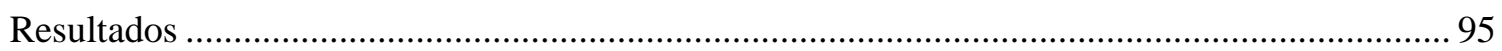

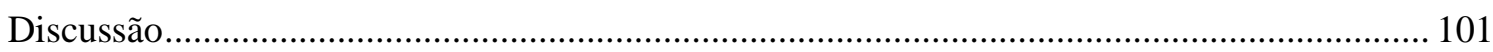

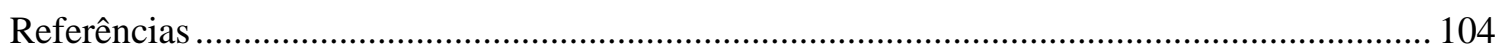

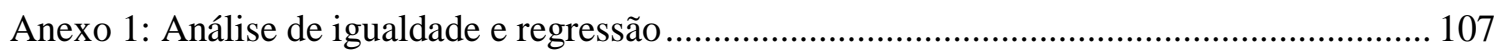

Anexo 2: Análise de equidade - pilar qualidade do ensino (Parte 1) .......................................... 108

Anexo 2: Análise de equidade - pilar qualidade do ensino (Parte 2) ....................................... 109

Anexo 2: Análise de equidade - pilar tempo de aprendizado ........................................................ 110

Anexo 2: Análise de equidade - pilar recursos escolares........................................................... 111

\section{Manuscrito 4}

ESTIMAÇÃO DOS EFEITOS DOS PILARES EDUCACIONAIS SOBRE O DESEMPENHO:

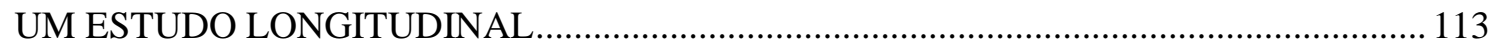

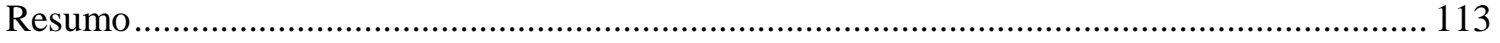

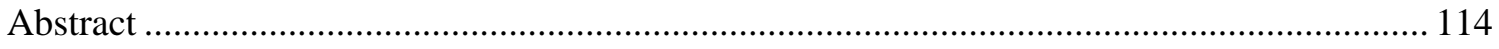

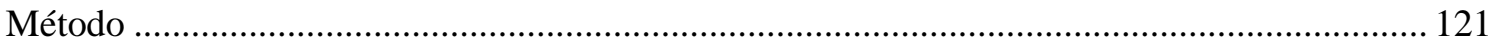

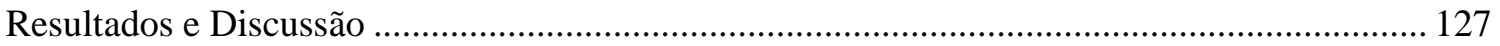

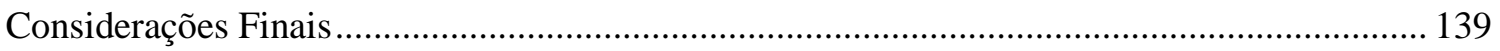

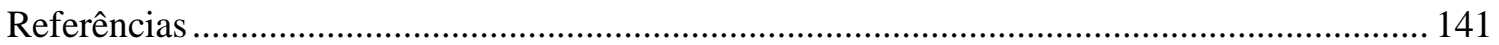




\section{Agradecimentos}

Ao meu pai pela dedicação e esforço de uma vida para fazer com que eu e meus irmãos nos tornássemos boas pessoas. Vou sempre me lembrar do meu pai acordando de madrugada para trabalhar e permitir que eu estudasse. Essa conquista, eu dedico a ele. Sinto que não pude compensá-lo por tudo o que fez por mim, mas minha gratidão é eterna.

À minha mãe pelo amor, incentivo e apoio incondicional. Sempre ao meu lado, me dando suporte e me fazendo acreditar que sou capaz. Mãe, essa conquista é tanto minha quanto sua.

Ao meu amado esposo, Ênio, pela compreensão e apoio durante esse período. Não foi fácil conciliar trabalho, casa, família e doutorado. Sem a sua ajuda com certeza eu não teria conseguido. Agradeço ainda por ter me escolhido e por me fazer feliz.

Aos meus irmãos, cunhadas e sobrinhos que nos momentos de minha ausência dedicados ao estudo, sempre fizeram entender que o futuro é feito a partir da constante dedicação no presente.

Ao meu orientador, professor Laros, pela disponibilidade, amizade, apoio e ensinamentos. Com você, aprendi mais do que fazer pesquisa, tive um exemplo de pessoa. Como ouvi do educador José Pachecco: "Professor não ensina aquilo que diz. Transmite aquilo que é". Eu tive a grata satisfação de ser sua aluna.

Aos membros da banca pelas contribuições em relação à tese. Especial agradecimento ao professor Francisco Soares pelo apoio no contato com o professor Willms, o que possibilitou a realização do meu estágio sanduíche no Canadá.

Aos pesquisadores e estudantes do Canadian Research Institute for Social Policy (CRISP) pelo suporte durante o período em que estive no Canadá, pelos convites para tomar um café e pelas discussões acadêmicas. Em especial, agradeço aos professores 
Willms, Lucia e Robert por terem me recebido tão bem e por terem se disposto a me orientar.

À CAPES (Coordenação de Aperfeiçoamento de Pessoal de Nível Superior) pela concessão da bolsa e pelo apoio durante o período de estágio sanduíche.

À Ellen por ter me recebido em sua casa e por ter me ajudado a enfrentar a saudade do Brasil. O estágio sanduíche também foi uma oportunidade para estabelecer uma grande amizade.

Aos professores do PSTO e aos colegas do grupo de pesquisa - Luís, Fabiana, Felipe, Daniel, Renata, Alexandre, Talita e Gina - pelo companheirismo e apoio. Um agradecimento especial ao Luís pela leitura crítica da minha tese, pelas sugestões e discussões. Foi um prazer ter orientação compartilhada com você, o que nos rendeu boas publicações e muitas que ainda virão.

Aos meus amigos do INEP que me apoiaram e incentivaram, principalmente no início do Doutorado quando eu ainda trabalhava lá. Um agradecimento especial a Patrícia pela parceria, amizade e revisão da minha tese.

Aos meus amigos da Geekie, pela parceria e compreensão. Nessa reta final, o apoio de vocês foi essencial para que eu conseguisse finalizar a tese. Sassaki, obrigada por acreditar em mim e esperar o meu retorno do Canadá. Maroja, obrigada pelo companheirismo e pelo ombro amigo.

Aos amigos e familiares que acompanharam a minha jornada e estivem sempre presentes em minha vida. 


\section{Resumo Geral}

O cerne desta tese de doutorado está no desafio da promoção de uma educação de qualidade e na concepção de que a escola é o principal elemento para que isso se concretize. A crença de que a escola pode fazer a diferença na formação do estudante tem norteado estudos desde a década de 1960, na busca de compreender o que torna a escola eficaz, delineando a área conhecida como educational effectiveness research (EER). Essa área, incialmente focada na eficácia e no efeito-escola, também engloba estudos referentes à identificação de fatores associados ao desempenho, à procura de métodos que buscam a melhoria da escola e à promoção da igualdade e da equidade. Neste contexto e considerando a relevância de garantir uma educação para todos, o objetivo geral desta tese foi avaliar o sistema educacional brasileiro em termos de o quanto ele tem proporcionado equidade e igualdade, além de identificar fatores escolares que podem contribuir para uma maior eficácia escolar. Para alcançar esse objetivo geral, no Estudo 1 foi realizada uma revisão sistemática das publicações brasileiras entre 2000 e 2013 na área de eficácia escolar, a fim de analisar os modelos teóricos e as variáveis (de contexto, input, processo e output) utilizados, bem como confrontar os resultados brasileiros com achados estrangeiros. A partir dessa revisão, verificou-se a necessidade de se utilizar um aporte teórico mais sólido, de se construir medidas que possam ser acompanhadas ao longo dos anos e de se investir em estudos longitudinais. Nesse sentido, no Estudo 2 buscou-se construir medidas válidas, a partir dos dados da Prova Brasil 2009, relativas à escola, que pudessem subsidiar a avaliação de igualdade, equidade e eficácia, propondo uma nova abordagem teórica como base: o modelo de prosperidade educacional. Esse modelo, tendo como suporte os achados da área de EER e a teoria do desenvolvimento, propõe quatro pilares para a garantia do sucesso educacional no final do ensino fundamental: (1) contexto inclusivo, (2) qualidade de ensino, (3) tempo de aprendizado e (4) recursos escolares. Além de construir medidas para esses pilares, o Estudo 2 apontou dificuldades do sistema educacional brasileiro em prover recursos escolares e garantir a qualidade de ensino. No Estudo 3, as medidas construídas no Estudo 2 foram utilizadas para analisar o quanto o sistema educacional brasileiro promove condições igualitárias e equânimes para estudantes de diferentes níveis socioeconômicos. Essa análise foi realizada em termos de chance de sucesso acadêmico e chance de acesso a recursos escolares. Os resultados apontaram disparidades de desempenho e diferenças na distribuição dos recursos, sendo, em geral, em desfavor dos estudantes pobres. Após a verificação dessas diferenças, realizou-se, por fim, o Estudo 4. Nele buscou-se compreender, a partir de análise transversal e longitudinal, a relação entre os pilares do modelo de prosperidade educacional e o desempenho. A análise transversal demonstrou o potencial dos pilares educacionais em contribuir com o desempenho e a análise longitudinal, adicionalmente, indicou pequena variabilidade no desempenho e nos pilares educacionais ao longo dos anos.

Palavras-chave: eficácia escolar; igualdade; equidade; pilares educacionais. 


\section{General Abstract}

The core of this doctoral thesis is in the challenge to promote quality education and in the conception that the school is the main element for this to happen. The belief that the school can make a difference in the formation of the student has guided studies since the 1960s in the search to understand what makes a school effective, outlining the area known as educational effectiveness research (EER). This area, initially focused on effectiveness and school effect, also includes studies on the identification of factors associated with academic performance, on the methods that seek to improve the school and on the promotion of equality and equity. In this context and considering the importance of ensuring education for all, the general objective of this thesis was to evaluate the Brazilian educational system in terms of how much it has provided equity and equality, and to identify school factors that can contribute to greater school effectiveness. To achieve this overall objective, in Study 1 a systematic review of Brazilian publications between 2000 and 2013 in the area of school effectiveness was performed in order to identify the theoretical models and variables used (context, input, process and output) and compare these results with those found in other countries. Based on this review, various needs were identified: for a more solid theoretical foundation, for the construction of measures that can be accompanied over the years, and for the development of longitudinal studies. In this context, in Study 2, we sought to build valid measures using data from Prova Brasil 2009, related to school, that could support the evaluation of equality, equity and effectiveness, proposing a new theoretical approach based on the educational prosperity model. This model, supported by the findings of EER area and the theory of development, proposes four pillars to ensure educational success at the end of elementary school: (1) inclusive context, (2) quality of instruction, (3) learning time and (4) school resources. In addition to construct measures for each of these pillars, Study 2 indicated difficulties of the Brazilian educational system to provide educational resources and ensure educational quality. In Study 3, the measures built in Study 2 were used to analyze how the Brazilian educational system promotes equal and equitable conditions for students of different socioeconomic levels. This analysis was performed in terms of chance of academic success and chance of access to school resources. The results showed performance gaps and differences in the distribution of resources, mainly at the disadvantage of poor students. Lastly, after the verification of differences, Study 4 was conducted. Using transverse and longitudinal analyses, this last study had the objective to investigate the relationship between the pillars of the educational prosperity model and academic performance. The cross-sectional analysis showed the potential of the educational pillars in the prediction of academic performance, while the longitudinal analysis indicated little variability in academic performance and in the educational pillars over the years.

Keywords: school effectiveness; equality; equity; educational pillars. 


\section{Lista de Siglas}

\begin{tabular}{|c|c|}
\hline ANOVA & Análise de Variância \\
\hline ANRESC & Avaliação Nacional do Rendimento Escolar \\
\hline CAEd & Centro de Avaliação da Educação da UFJF \\
\hline CRISP & Canadian Research Institute for Social Policy \\
\hline EER & Educational Effectiveness Research \\
\hline $\mathrm{EF}$ & Ensino Fundamental \\
\hline EM & Ensino Médio \\
\hline EP & Erro padrão \\
\hline GAME & Grupo de Avaliação e Medidas Educacionais da UFMG \\
\hline GERES & Estudo Longitudinal da Geração Escolar \\
\hline ICC & Intraclass Correlation \\
\hline INEP & Instituto Nacional de Estudos e Pesquisas Educacionais Anísio Teixeira \\
\hline IRT & Item Response Theory \\
\hline LAED & Laboratório de Avaliação da Educação da PUC-Rio \\
\hline LOED & Laboratório de Observação e Estudos Descritivos da UNICAMP \\
\hline MEC & Ministério da Educação e Cultura \\
\hline NEPO & Núcleo de Estudos de População da UNICAMP \\
\hline NSE & Nível Socioeconômico \\
\hline OREALC & Oficina Regional de Educação para a América Latina e o Caribe \\
\hline PERCE & Primeiro Estudo Regional Comparativo e Explicativo \\
\hline PIRLS & Progress in Reading Literacy Study \\
\hline PISA & Programme for International Student Assessment \\
\hline
\end{tabular}


PNE Plano Nacional de Educação

PUC-Rio Pontifícia Universidade Católica do Rio de Janeiro

SAEB Sistema Nacional de Avaliação da Educação Básica

SERCE Segundo Estudo Regional Comparativo e Explicativo

SES Socioeconomic Status

SIMAVE Sistema Mineiro de Avaliação da Educação Pública

SPSS Statistical Package for the Social Science

TERCE Terceiro Estudo Regional Comparativo e Explicativo

TIMSS Trends in International Mathematics and Science Study

TRI Teoria de Resposta ao Item

UEMS Universidade Estadual do Mato Grosso do Sul

UF Unidade da Federação

UFBA Universidade Federal da Bahia

UFJF Universidade Federal de Juiz de Fora

UFMG Universidade Federal de Minas Gerais

UNESCO Organização das Nações Unidas para a Educação, a Ciência e a Cultura

UNICAMP Universidade de Campinas

VCR Video Cassette Recorder

2PL Two-Parameter Logistic model 


\section{Lista de Figuras}

Manuscrito 1: Estudos brasileiros sobre eficácia escolar: uma revisão de literatura.

Figura 1: Modelo integrado de avaliação da eficácia escolar................................... 25

Figura 2: Quantidade de artigos publicados na área de eficácia escolar por ano no

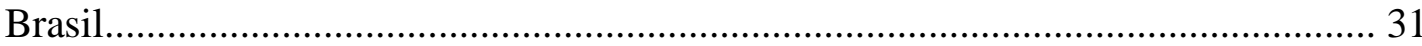

Manuscrito 2: Pillars of good education: Analyzing the Brazilian school system.

Sem Figuras

Manuscrito 3: Avaliação da igualdade e da equidade do sistema educacional público brasileiro.

Figura 1: Modelo de avaliação de igualdade e equidade de um sistema educacional. 87

Figura 2: Gráfico de regressão: nível socioeconômico e desempenho em matemática da escola 99

Manuscrito 4: Estimação dos efeitos dos pilares educacionais sobre o desempenho: um estudo longitudinal.

Sem Figuras 


\section{Lista de Tabelas}

Manuscrito 1: Estudos brasileiros sobre eficácia escolar: uma revisão de literatura.

Tabela 1: Autores, ano de publicação, base de dados, etapa de ensino e tipo de análise dos relatos de pesquisa sobre eficácia escolar no Brasil.

Tabela 2: Fatores/Variáveis contextuais utilizados nos estudos brasileiros de eficácia escolar e sua relação com desempenho

Tabela 3: Fatores/Variáveis de input utilizados nos estudos brasileiros de eficácia escolar e sua relação com desempenho.

Tabela 4: Fatores/Variáveis de processo utilizados nos estudos brasileiros de eficácia escolar e sua relação com desempenho

Manuscrito 2: Pillars of good education: Analyzing the Brazilian school system.

Table 1: Description of the measures used to represent each pillar of the Educational Prosperity model

Table 2: Description and parameters of the items - scale of pedagogical practices

Table 3: Interpretation and percentage of school per level - scale of pedagogical practices 65

Table 4: Frequency of answers, missing data, skewness and kurtosis - quality of instruction variables

Table 5: Frequency of answers, missing data, skewness and kurtosis - learning time variables 70

Table 6: Description and parameters of the items - scale of infrastructure conditions

Table 7: Interpretation and percentage of school per level - scale of infrastructure conditions.................................................................. 71

Table 8: Description and parameters of the items - scale of equipment ............ 72

Table 9: Interpretation and percentage of school per level - scale of equipment..... 73

Table 10: Description and parameters of the items - scale of human resources problems............................................................. 73

Table 11: Interpretation and percentage of schools per level - scale of human resources problems...................................................... 74

Table 12: Description and parameters of the items - scale of socioeconomic level 
Table 13: Interpretation and percentage of students per level - scale of

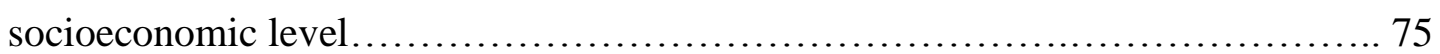

Table 14: Description and parameters of the items - scale of family support........ 76

Table 15: Interpretation and percentage of students per level - scale of family support.............................................................. 76

Table 16: Description and parameters of the items - scale of community

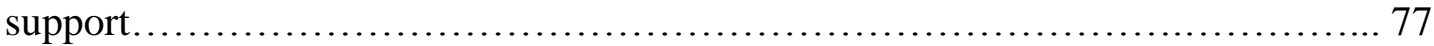

Table 17: Interpretation and percentage of schools per level - scale of community support.....

Table 18: Frequency of answers, missing data, skewness and kurtosis - attainment variables 78

Manuscrito 3: Avaliação da igualdade e da equidade do sistema educacional público brasileiro.

Tabela 1: Descrição das variáveis utilizadas no estudo. 93

Tabela 2: Média e desvio-padrão do desempenho em matemática por nível socioeconômico 96

Tabela 3: Percentual de estudantes que atingem o nível adequado de proficiência, razão de chance de sucesso e chance relativa 98

Tabela 4: Percentual de estudantes com acesso a recursos escolares, chance relativa e razão de chance de acesso

Manuscrito 4: Estimação dos efeitos dos pilares educacionais sobre o desempenho: um estudo longitudinal.

Tabela 1: Descrição das variáveis: desempenho escolar, suporte familiar/ comunidade e nível socioeconômico 122

Tabela 2: Descrição das variáveis representantes dos pilares 1 e 2 do modelo de prosperidade educacional

Tabela 3: Descrição das variáveis representantes dos pilares 3 e 4 do modelo de prosperidade educacional

Tabela 4: Modelos 0,1 e 2 da análise multinível - Prova Brasil 2009.

Tabela 5: Modelos 3,4 e 5 da análise multinível - Prova Brasil 2009.

Tabela 6: Modelos 6 e 7 da análise multinível - Prova Brasil 2009.

Tabela 7: Modelos 0,1 e 2 da análise multinível - Prova Brasil 2007, 2009, 2011 e 2013

Tabela 8: Modelo 3 e modelo final da análise multinível - Prova Brasil 2007, 2009, 2011 e 2013 


\section{Apresentação}

O maior desafio de um sistema educacional é conseguir prover uma educação adequada e de qualidade para todas as crianças, jovens e adultos. Essa educação de qualidade, direito fundamental de todas as pessoas, além de ser eficaz e eficiente, deve respeitar direitos, ser relevante, pertinente e equânime (UNESCO, 2008). Considerando a magnitude do Brasil, em conjunto com a sua diversidade e desigualdade, esse desafio torna-se ainda mais difícil de ser alcançado.

Há poucos anos, o governo brasileiro ainda enfrentava uma questão anterior, a de possibilitar acesso à escola. Sobretudo para o ensino fundamental, essa já não é mais uma questão de primeiro plano. Com isso, a problemática central passa a ser a qualidade da educação que tem sido fornecida pelas escolas, para que o pleno exercício do direito de aprender se concretize. Como afirmado por Soares e Andrade (2006), o acesso à escola está garantido, mas apenas alguns terminam a educação básica com níveis de desempenho adequado.

O direito à educação não consiste somente em colocar as crianças na escola, implica dar condições para um desenvolvimento físico, social, intelectual e crítico. Enfim, é preciso possibilitar um desenvolvimento pleno que dê subsídios para o contínuo aprendizado. É preciso garantir uma aprendizagem transformadora, tendo em vista o “direito de ser mais" (Freire, 2014).

Na busca por contribuir para a superação desse desafio é que esta tese se insere. Entende-se que a academia, sempre quando possível, deve estar alinhada às demandas da sociedade, de modo a contribuir para que as ações de educadores e políticos sejam certeiras e eficazes. Na ausência de dados, estudos e resultados, as ações tornam-se meramente intuitivas ea educação não deve ser uma área de atuação intuitiva. Nessa conjuntura, entre 
tentativas e erros, milhares de jovens podem ser prejudicados por não terem tido uma educação de qualidade.

Torna-se, então, cada vez mais necessário, levantar dados, avaliar e monitorar programas existentes, entender como o processo de aprendizagem ocorre e identificar os fatores que influenciam esse processo a fim de subsidiar discussões e ações educativas. Essa concepção está alinhada com o Plano Nacional da Educação (PNE) aprovado em 2014 e que orienta as ações do governo federal para os próximos dez anos (MEC, 2014). Neste plano, o primeiro grupo de metas busca a garantia do direito à educação básica com qualidade, o que envolve a garantia do acesso, a ampliação da escolaridade e a melhoria do fluxo escolar e da aprendizagem. Um segundo grupo de metas tem como propósito a redução das desigualdades e a valorização da diversidade. Em conjunto, esses dois grupos de metas indicam a relevância da promoção da qualidade com equidade. No PNE, apontase ainda, como estratégia para cumprimento das metas, a necessidade de se fortalecer os sistemas de monitoramento e avaliação.

Nesse contexto, as pesquisas aqui apresentadas tiveram como objetivo trazer contribuições acadêmicas e sociais para a educação. Teve-se como objetivo geral avaliar o sistema educacional brasileiro em termos de o quanto ele tem proporcionado equidade e igualdade, além de identificar fatores escolares que podem contribuir para uma maior eficácia escolar. Esse objetivo embasa-se na tese de que, em busca de um sistema educacional de qualidade que garanta o direito de aprender a todos e a cada um, é preciso avaliar os níveis atuais de equidade e igualdade, bem como identificar quais fatores escolares podem garantir uma maior eficácia.

A igualdade pode ser definida em termos de todos os estudantes obterem resultados de aprendizado semelhantes, independentemente de etnia, gênero, status social ou econômico. Portanto, num sistema igualitário, o aprendizado é garantido a todos, sem 
distinção, permitindo que todos alcancem os níveis adequados esperados. Para a igualdade de resultados é necessário garantir a oportunidade de acesso a recursos e processos escolares. É nesse contexto que se insere o termo equidade, o qual está relacionado ao senso de justiça e à inclusão. Condições diferentes exigirão ações e esforços diferentes, o que pode estar relacionado a políticas compensatórias ou à alocação diferenciada de recursos. Por fim, o termo eficácia é atribuído àquelas escolas que conseguem garantir resultados superiores aos proporcionados pelas escolas em semelhantes condições. Esses três termos têm em comum o conceito de qualidade da educação e estão contemplados nas pesquisas da área de eficácia escolar. Para a avaliação da igualdade, equidade e eficácia no sistema educacional brasileiro, foram desenvolvidos quatro estudos.

No Estudo 1, objetivou-se realizar um levantamento das pesquisas sobre eficácia escolar no Brasil, além de sistematizar os resultados nacionais e confrontar com os achados estrangeiros. Almejava-se, portanto, delinear um aporte teórico sólido que pudesse orientar toda a execução dos demais estudos.

O Estudo 2 guarda contribuições relevantes do grupo de pesquisadores do Canadian Research Institute for Social Policy (CRISP) da Universidade de New Brunswick, em especial do Prof. PhD. J. Douglas Willms. No período em que estive no Canadá (estágio sanduíche), o grupo estava na fase de construção dos questionários para os países em desenvolvimento participantes do PISA, Programme for International Student Assessment. Para a construção desses questionários, o grupo estava em plena concepção do modelo de prosperidade educacional, que será a fundamentação teórica para o desenvolvimento das medidas. Foi a oportunidade de conhecer um modelo em eminência, que busca definir teoricamente os fatores essenciais para o sucesso educacional, diferenciando-se das tradicionais pesquisas de eficácia escolar que têm como foco a busca "do que funciona" de 
acordo com o modelo de input-processo-output (Creemers, Kyriakides, \& Sammons, 2010).

O modelo de prosperidade educacional define os elementos que precisam ser garantidos pela família, pela instituição (escola) e pela comunidade em cada etapa do desenvolvimento para o alcance do sucesso educacional. No que concerne à escola, quatro pilares são elencados como essenciais para os alunos que estão no fim da educação básica: qualidade de ensino, tempo de aprendizado, ambiente inclusivo e recursos escolares (Willms, Durepos, López Garcia, Laurie, \& Tramonte, 2015).

Esse modelo foi pensado para conceber os questionários do PISA for Development, avaliação destinada especificamente para os países em desenvolvimento. Essa abordagem teórica diferenciada fez-se necessária, uma vez que se percebeu que os questionários tradicionais do PISA não eram adequados para os países em desenvolvimento, cujo contexto socioeconômico é bastante específico. Portanto, como esse modelo é recente, há ainda poucas publicações sobre ele e nenhum estudo de validação do modelo foi executado. Entende-se que o modelo é promissor e pode contribuir para tornar a área de eficácia escolar mais teoricamente dirigida. Todavia, também é preciso cautela, uma vez que ainda não há evidências de sua efetividade. Esta tese é o primeiro estudo brasileiro que usa e busca validar essa abordagem teórica.

Portanto, tendo como base a experiência no Canadá, o Estudo 2 assume como base teórica o modelo de prosperidade educacional e busca-se, a partir dos dados da Prova Brasil, construir medidas escolares e contextuais compatíveis com esse modelo. Esse é um estudo intermediário que viabiliza a realização dos estudos posteriores de avaliação da igualdade, equidade e eficácia.

No Estudo 3, teve-se como objetivo avaliar a equidade e igualdade do sistema educacional brasileiro a partir da análise da diferença de desempenho acadêmico e da 
diferença de acesso aos pilares do modelo de prosperidade educacional de acordo com a condição socioeconômica do estudante. Essa é uma forma de verificar se a educação tem sido oportunizada a todos, sem distinção. A análise poderia ser realizada considerando grupos por sexo, etnia, região etc. No entanto, optou-se pela análise por nível socioeconômico porque a educação carrega o estigma da emancipação, de que ela pode diminuir diferenças sociais. Isso perpassa necessariamente pela igualdade de oportunidade e por políticas compensatórias.

No Estudo 4, buscou-se verificar de modo transversal e longitudinal o efeito dos pilares da escola sobre o desempenho e se esses pilares contribuem para explicar porque algumas escolas são mais eficazes do que outras. Esse estudo diferencia-se dos estudos anteriores na área de eficácia escolar ao investir numa nova abordagem teórica para inclusão de variáveis no modelo, mas, sobretudo, por realizar uma análise de medidas repetidas com os dados da Prova Brasil, o que permite acompanhar longitudinalmente as escolas, bem como permite distinguir melhor as mudanças de desempenho decorrentes de mudanças contextuais, daquelas provenientes de fatores intrinsecamente escolares.

Os quatro estudos representam o progresso da tese, sendo os dois primeiros meio (revisão teórica e construção de medidas) para a adequada avaliação de igualdade, equidade e eficácia. Espera-se que esses estudos possam mostrar um retrato do sistema educacional brasileiro, mas, sobretudo, possam contribuir para o avanço da área e para a reflexão de como proporcionar uma educação de qualidade. 


\section{Referências}

Creemers, B. P. M., Kyriakides, L., \& Sammons, P. (2010). Methodological advances in educational effectiveness research. London, New York: Routledge.

Freire, P. (2014). A pedagogia do oprimido. São Paulo: Mercado Cultural.

MEC (2014). Planejando a próxima década: Conhecendo as 20 metas do Plano Nacional de Educação. Brasília: Autor.

Soares, J. F., \& Andrade, R. J. (2006). Nível socioeconômico, qualidade e equidade das escolas de Belo Horizonte. Ensaio: Avaliação e Políticas Públicas Educacionais, 14(50), 107-126.

UNESCO (2008). Educação de qualidade para todos: Um assunto de direitos humanos. Brasília: Autor.

Willms, J. D., Durepos, D., López García, A. Y., Laurie, R., \& Tramonte, L. (2015). Design and composition of contextual questionnaires for the PISA for development study. Fredericton, NB: The Learning Bar Inc. 
Manuscrito 1

ESTUDOS BRASILEIROS SOBRE EFICÁCIA ESCOLAR:

UMA REVISÃO DE LITERATURA

\section{Resumo}

Na busca por uma educação de qualidade, vários estudos foram iniciados na década de 1960 a fim de avaliar se as escolas contribuem significativamente na formação dos estudantes, o que levou ao surgimento da área de eficácia escolar. Neste estudo, tem-se como objetivo principal realizar uma revisão sistemática da literatura brasileira na área de eficácia escolar. Foram analisados 30 artigos publicados em periódicos científicos entre 2000 e 2013. Foi possível identificar efeitos consistentes de fatores como nível socioeconômico, etnia e atraso escolar sobre o desempenho escolar, bem como ficaram evidentes dificuldades de definição e mensuração de fatores intraescolares. Ressalta-se também que problemas de igualdade e equidade precisam ser melhor investigados. Os resultados demonstram similaridades com a literatura estrangeira e ajudam a nortear estudos futuros na área de eficácia escolar.

Palavras-chaves: eficácia escolar; efeito escola; desempenho escolar; avaliação escolar. 


\begin{abstract}
In search for quality education, several studies were initiated in the decade of 1960 with the objective to evaluate to what extent schools contribute to the formation of their students. These studies have led to the emergence of the area of school effectiveness. The main objective of this study was to accomplish a systematic review of the Brazilian literature in the area of school effectiveness. Thirty articles published between 2000 and 2013 in Brazilian scientific journals were analyzed. Solid effects of factors like socioeconomic level, race and school delay on school performance were identified. In addition, difficulties related to the definition and measurement of intra-school factors became evident. It should be emphasized that problems of equality and equity need to be investigated better. The results showed similarities with the results of the scientific literature of other countries and can guide future studies in the area of school effectiveness. Keywords: school effectiveness; school performance; educational assessment.
\end{abstract}


É fascinante o enigma que envolve descobrir porque algumas escolas têm maior capacidade do que outras de proporcionar melhores resultados acadêmicos (Lee, 2008). Essa afirmação provoca a reflexão sobre a importância dos contextos nos quais os alunos se desenvolvem para o progresso educacional. Isso porque, além da potencialidade individual e outros aspectos que o aluno traz consigo, fatores contextuais exercem uma forte influência no aprendizado.

Tendo como meta a promoção de uma educação de qualidade, vários estudos foram iniciados na década de 1960, a fim de avaliar se as escolas contribuem significativamente na formação dos estudantes. Em específico, buscava-se identificar quais fatores escolares contribuem para uma maior eficácia escolar. Termo este utilizado para se referir à capacidade de a escola contribuir para que seus alunos alcancem resultados para além do esperado, considerando características contextuais e escolares (Mortimore, 1991). Para Reynolds, Teddlie, Creemers, Scheerens e Townsend (2000), a área de eficácia escolar busca compreender e analisar o papel da escola por meio de estudos quantitativos e qualitativos sobre o efeito escola (school effects), escolas efetivas ou eficazes (effective schools) e formas de melhoria da escola (school improvement).

Nesses quase 50 anos de pesquisas na área de eficácia escolar, muito já foi produzido e discutido. Neste artigo, tem-se como objetivo principal realizar uma análise da produção brasileira na área de eficácia escolar, buscando contrapor com a literatura estrangeira, delineando lacunas que permitam subsidiar uma agenda de pesquisa.

\section{Histórico dos estudos sobre eficácia escolar.}

O relatório publicado por Coleman et al. (1966) é tido como um marco na área de eficácia escolar. Nele, os pesquisadores afirmaram que cerca de $90 \%$ da variação no desempenho acadêmico é explicada pelas condições socioeconômicas dos alunos e de suas 
famílias e que diferenças entre as escolas representam uma interferência muito pequena no desempenho acadêmico. Dessa forma, o relatório impactou toda a comunidade acadêmica ao apontar que escolas têm pouca influência no desempenho de um aluno, quando controlados o contexto socioeconômico e os antecedentes sociais.

O impacto gerado por esses resultados impulsionou uma série de outros estudos que buscaram compreender se as escolas realmente podem fazer a diferença (Edmonds, 1979; Mortimore, Sammons, Stoll, Lewis, \& Ecob, 1988; Rutter, Maughan, Mortimore, Ouston, \& Smith, 1979). Essa reação delineou a constituição da área de eficácia escolar e contribuiu para o avanço significativo nas discussões sobre equidade e igualdade educacional, bem como no entendimento de como fatores escolares e extraescolares interferem no desempenho acadêmico.

Ao surgir, em reação aos resultados apontados pelo Relatório Coleman, a área de eficácia escolar desenvolve-se inicialmente em um clima reativo, muito focado no papel da escola e na busca incessante por encontrar fatores que expliquem a eficácia educacional. Essas características iniciais exerceram forte influência na produção da área tanto em termos teóricos, quanto metodológicos.

Em termos teóricos, ainda no final do século XX, os estudos sobre eficácia escolar são criticados por consistir em numerosos estudos empíricos, carentes de uma teoria sólida que pudesse ser verificada (Van Den Eeeden, Hox, \& Hauer, 1990). Apenas mais recentemente é que se tem constituído uma teoria da área que visa explicar a associação e a dinâmica entre as variáveis (Reynolds, Sammons, Fraine, Townsend, \& Damme, 2011).

Ao analisar milhares de estudos a partir de uma meta-análise, Teodorovic (2009) identificou que a maioria dos estudos ao longo do desenvolvimento da área pode ser categorizada em três grupos, conforme seus paradigmas teóricos: (1) os estudos de inputoutput; (2) os estudos de eficácia escolar e (3) os estudos de eficácia docente. 
A fundamentação teórica dos estudos de input-output é a de que os inputs da escola (recursos humanos, infraestrutura, verba) são os determinantes para um bom desempenho. Essa concepção norteou os estudos iniciais na área de monitoramento de sistema e, de forma geral, buscava demonstrar o efeito de fatores econômicos, organizacionais e de estrutura sobre os resultados da escola. Carvallo-Pontón (2010) complementa que esses foram os primeiros estudos na área, nos quais se utilizavam técnicas de regressão linear e, muitas vezes, havia alta colinearidade entre as variáveis.

Os resultados dos estudos de input-output, em geral, foram contraditórios e sugeriam efeitos fracos dos inputs. Para Willms (1992), vários fatores contribuíram para esses resultados: (1) ausência de grandes diferenças nas políticas e práticas adotadas entre as escolas; (2) ausência de especificação de efeitos entre níveis; (3) limitação dos estudos a fatores fáceis de serem definidos e medidos, sendo que os principais processos que afetam a escolarização são de difícil definição e mensuração; e (4) não abrangência de fatores contextuais nos estudos, com isso não se distinguiam os efeitos escolares dos contextuais.

Além disso, os estudos de input-output foram criticados por não apresentarem fatores que pudessem ajudar os educadores a melhorar as escolas. Nesses estudos, as escolas eram tratadas como "caixas pretas", investigando-se apenas os insumos e os produtos. Na tentativa de desvendar as "caixas pretas", surgem os estudos de eficácia escolar propriamente ditos.

A diferença em relação aos estudos anteriores é o foco nos processos escolares que ocorriam dentro da "caixa-preta" da escola (Alves \& Soares, 2007a). Esses estudos de eficácia escolar concebem que o desempenho do aluno é determinado por fatores escolares e processuais, tais como: a presença de um bom líder, foco nos objetivos a serem alcançados, altas expectativas, cooperação, clima acadêmico e monitoramento constante (Teodorovic, 2009). 
Os estudos de eficácia escolar passam a utilizar um modelo aprimorado do antigo input-output, uma vez que reconhecem a estrutura multinível e buscam separar recursos de entrada dos fatores processuais (Willms, 1992). O objetivo era estimar a magnitude dos efeitos escolares, entendidos como a capacidade de as escolas influenciarem o desenvolvimento do aluno, além de trazer mais informações processuais que permitissem melhor compreender as razões de algumas escolas terem melhores resultados do que outras (Carvallo-Pontón, 2010). Engloba-se, portanto, estudos que buscam estimar o efeito escola (nas suas várias dimensões: valor agregado, eficácia diferencial, equidade social), identificar fatores associados ao desempenho, identificar características que tornam umas escolas mais eficazes do que outras e encontrar meios de promoção de mudanças na escola.

Nesse contexto de compreender melhor as dinâmicas intraescolares, surgem também as pesquisas que buscam explorar o efeito de diferentes tipos de práticas de ensino sobre o desempenho acadêmico. Esses estudos concentraram-se em verificar os comportamentos dos professores e as práticas de sala de aula utilizadas para explicar o alcance de um bom desempenho (Teodorovic, 2009).

Se por um lado os estudos de eficácia docente (instructional effectiveness) podem ser classificados como pertencentes a um paradigma específico, por outro, as características de ensino podem também ser utilizadas como fatores processuais nos estudos de eficácia escolar. A diferença mais marcante quando características processuais de ensino são incorporadas nos modelos de eficácia escolar é o acréscimo de outro nível de análise relativo ao professor ou à turma.

Ao sistematizar a produção na área, Scheerens (1990) propõe o modelo básico de funcionamento da escola. Esse modelo busca integrar e ser uma estrutura de referência das variáveis que exercem efeito sobre o desempenho escolar (output), categorizando-as em contextuais, de input e de processo. A Figura 1 representa a proposta do modelo integrado. 


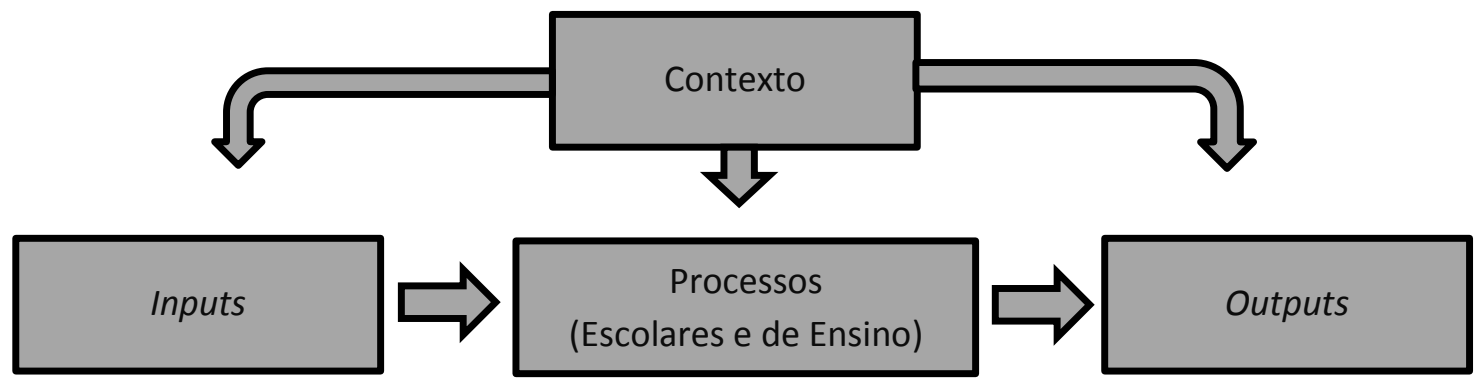

Figura 1

Modelo integrado de avaliação da eficácia escolar (Scheerens, 1990).

No modelo apresentado, os fatores contextuais são aqueles fatores extraescolares que impactam no processo educativo, mas que a escola possui pouco ou nenhum gerenciamento sobre eles. Scheerens (1990) menciona, por exemplo, gestão administrativa, a localização da escola (rural ou urbana) e tamanho da escola. Soares (2007) cita outros possíveis fatores extraescolares: valores sociais, políticas públicas, recursos, gestão do sistema pelas secretarias educacionais e características socioeconômicas e culturais da comunidade.

A segunda parte do modelo integrado corresponde às ações intraescolares, que envolvem: input, processo e output. Entende-se como fatores de input aqueles que são insumos para ocorrência do processo educativo. Scheerens (1990) cita como exemplos a experiência/formação do professor, a experiência do diretor e o suporte familiar.

Quanto aos fatores processuais, a literatura inicial das pesquisas de eficácia escolar sugeria um modelo de cinco fatores que combinados tornariam uma escola eficaz: (1) liderança educacional forte; (2) ênfase na aquisição das habilidades básicas; (3) ambiente organizado e seguro; (4) altas expectativas sobre o desenvolvimento dos estudantes; e (5) avaliação frequente do progresso dos estudantes (Odden, 1982; Scheerens, 2000). Todavia, esses estudos iniciais foram muito criticados no que concerne ao método empregado. 
Estudos posteriores, mais robustos metodologicamente, foram conduzidos, o que ampliou a lista de características das escolas eficazes. Sammons, Hillman e Mortimore (1995), na intenção de fornecer um resumo das evidências de pesquisas de eficácia escolar, identificaram 11 fatores-chave:

1. Liderança profissional firme, objetiva e com enfoque participativo.

2. Objetivos e visões compartilhadas.

3. Um ambiente de aprendizagem ordenado e atraente.

4. Concentração no ensino e na aprendizagem (ênfase acadêmica e foco no desempenho).

5. Ensino e objetivos claros.

6. Altas expectativas e fornecimento de desafios.

7. Incentivo positivo.

8. Monitoramento do progresso.

9. Direitos e responsabilidades dos alunos (alta autoestima do aluno, oportunidade de assumir posições de responsabilidade, controle dos trabalhos).

10. Parceria casa-escola (estabelecimento de relação de apoio e cooperação em prol da aprendizagem).

11. Uma organização orientada à aprendizagem (desenvolvimento de formação de pessoal na escola).

Os autores ressaltaram, no entanto, que esses fatores não devem ser considerados de modo independente. Várias associações entre eles podem ajudar a fornecer um melhor entendimento de prováveis mecanismos de eficácia. Ressalta-se ainda que esses fatores foram extraídos de pesquisas de etapas de ensino distintas, considerando contextos e desempenhos específicos. 
Quanto ao output, a maioria das pesquisas utiliza-se exclusivamente de resultados cognitivos dos alunos na área de leitura e matemática (Sammons, Hillman, \& Mortimore, 1995). O output deve ser o resultado esperado da escola: alunos bem formados. Certamente, a formação adequada do aluno implica mais do que a formação acadêmica cognitiva. Todavia, a ausência de medidas sistemáticas de outros aspectos que complementam a formação escolar faz com que as pesquisas utilizem majoritariamente como output o desempenho cognitivo.

Em consonância com os avanços teóricos, a análise das produções na área de eficácia escolar demonstra um sofisticado avanço metodológico. Inicialmente, alguns resultados controversos encontrados na literatura decorreram de dificuldades metodológicas que inflaram determinados resultados, uma vez que não utilizavam análises que consideram a estrutura hierárquica do sistema educacional. Por exemplo, procedimentos estatísticos tradicionais, tal como a análise de regressão múltipla, tratam os indivíduos de modo independente, não considerando a sua estrutura grupal, com isso as possíveis influências dessa estrutura são ignoradas (Goldstein, 2010; Hox, 2010).

Outra questão que tem sido cada vez melhor tratada é a dificuldade de se distinguir os componentes estáveis e instáveis, uma vez que a maior parte dos estudos de eficácia escolar é transversal. A fim de resolver essa questão, além do aumento de estudos longitudinais, técnicas de análises de medidas repetidas têm ganhado espaço nas pesquisas (Willms \& Raudenbush, 1989). Portanto, nota-se uma sofisticação metodológica na área, sendo que cada vez mais têm sido utilizadas: modelagem multinível, meta-análises, modelagem por equações estruturais, modelagem por curva de crescimento, além de pesquisas que empregam multimétodos.

De modo a sintetizar a história de desenvolvimento da área de eficácia escolar, Reynolds et al. (2011) a divide em cinco fases. A primeira fase é marcada por um conjunto 
de estudos que buscam demonstrar que as escolas fazem a diferença, em oposição ao chamado relatório Coleman. Já a segunda e a terceira fases (meados da década de 1980 e início da década de 1990) são marcadas por avanços metodológicos na busca de melhor estimar o efeito escola e pelas inúmeras tentativas de explorar as razões que explicam os diferentes efeitos entre escolas, respectivamente.

A quarta fase (final da década de 1990) é o período de internacionalização do campo de pesquisa e integração de resultados. É neste período que se iniciam as primeiras pesquisas no Brasil. As primeiras iniciativas brasileiras estão relacionadas ao início das avaliações em larga escala e os primeiros estudos foram publicados no início dos anos 2000. Para Soares (2007), as pesquisas brasileiras sobre eficácia escolar são incipientes, uma vez que o paradigma dominante de pesquisa educacional não privilegia esse tema.

A quinta e última fase (início dos anos 2000 e ainda corrente) inicia-se com os estudos que tentam analisar a eficácia escolar de forma dinâmica, compreendendo a educação como um conjunto de fatores que se relacionam. O modelo dinâmico busca explicar porque os sistemas educacionais funcionam diferenciadamente, baseando-se em quatro pressupostos: (1) tempo e oportunidade são variáveis do nível do aluno que estão diretamente relacionados ao sucesso; (2) ensino de qualidade e currículo são variáveis de processo que influenciam o tempo e a oportunidade de aprendizado; (3) por sua vez, ensino de qualidade, tempo e oportunidade são variáveis que sofrem influência de variáveis do nível da escola que podem contribuir na promoção desses fatores nas salas de aula; e, por fim, (4) o sucesso no desempenho também é determinado por fatores atitudinais, motivacionais e comportamentais relacionados ao estudante. Além disso, quatro aspectos operam na relação entre essas variáveis: consistência, coesão, constância e controle (Kyriakides, 2008). 
Embora os estudos sobre eficácia escolar tenham se aprimorado em termos de fundamentação teórica, ainda se fazem necessários estudos que sustentem tais fundamentações, o que demanda também aprimoramentos metodológicos. No entanto, para avançar é essencial consolidar os resultados encontrados até o momento, mesmo porque uma das críticas à área é que, devido ao seu crescimento rápido, ela aprendeu pouco com ela mesma (Reynolds et al., 2011). Nesse sentido, este estudo tem como objetivo revisar criticamente as produções brasileiras na área de eficácia escolar. Especificamente, busca-se analisar as concepções teóricas, os objetivos, as variáveis (de contexto, input, processo e output), os modelos de análise e os principais resultados encontrados.

Decidiu-se por realizar uma revisão da literatura brasileira para que seja possível confrontar os resultados nacionais com os destacados na literatura estrangeira, sobretudo porque os fatores associados ao desempenho escolar podem sofrer influência de características do sistema educacional sob análise e da realidade de determinado país. Além disso, a discussão dos resultados brasileiros à luz da literatura estrangeira permitirá delinear e propor pesquisas futuras para esta área, que ainda necessita de subsídios acadêmicos para crescer.

\section{Método}

Com a finalidade de analisar as produções sobre eficácia escolar no Brasil, foram levantados 30 artigos publicados em periódicos científicos entre 2000 e 2013. Esses artigos foram selecionados a partir de uma pesquisa bibliográfica realizada com as seguintes etapas e critérios:

(1) busca na base de dados da Scielo. Essa busca foi realizada com a utilização das palavras-chave: eficácia escolar (5 artigos), efeito escola (2 artigos), efeito-escola 
com a utilização de hífen (3 artigos), desempenho escolar (43 artigos). Dessa primeira busca, resultaram 52 artigos, pois houve a incidência de um artigo comum entre as palavras-chave eficácia escolar e desempenho escolar.

(2) leitura e classificação dos artigos. Para a inclusão do artigo nesta revisão de literatura necessariamente ele precisava abordar questões referentes a: estimação de efeito escola ou valor agregado; eficácia ou eficiência escolar; equidade e igualdade; identificação de fatores escolares que contribuem para a eficácia escolar ou métodos adequados para avaliação da eficácia escolar. Esse critério foi estabelecido tendo como base a definição proposta por Ferrão e Couto (2013, p. 137): “eficácia escolar designa a área de investigação científica em educação que é dedicada à estimação do efeito-escola (nas dimensões de valor agregado, eficácia diferencial e equidade social), à identificação de fatores que contribuem para que uma escola seja eficaz e abrange ainda o campo de trabalho cujo enfoque é a procura de métodos adequados e fiáveis para medir qualidade da escola”. Foram excluídos 36 artigos por não atenderem a esse critério.

(3) avaliação das referências bibliográficas dos 16 artigos classificados como adequados. Essa avaliação resultou na seleção de mais 11 artigos, dos quais dois foram excluídos por não se tratarem de relato de pesquisa ou de discussão sobre a literatura da área.

(4) avaliação das referências bibliográficas dos nove novos artigos agregados à seleção. Essa nova avaliação resultou na identificação de mais cinco artigos que se enquadravam nos requisitos desta pesquisa.

(5) avaliação das referências bibliográficas dos cinco novos artigos agregados à seleção. Nessa avaliação não foram encontradas novas referências. Encerrou-se, assim, a pesquisa bibliográfica. 
Para a análise dos artigos selecionados, optou-se por um procedimento padronizado para extração e classificação das informações referentes ao tipo de pesquisa (estudo teórico ou relato de pesquisa), à finalidade, à origem dos dados, ao tipo de análise e às variáveis utilizadas.

\section{Resultados e Discussão}

A Figura 2 apresenta a quantidade de artigos publicados por ano na área de eficácia escolar no Brasil. O ano em que ocorreu o maior número de publicação foi 2007 (n = 6). Apesar de não ser uma produção densa, houve a publicação de pelo menos um artigo por ano ao longo dos últimos 14 anos.

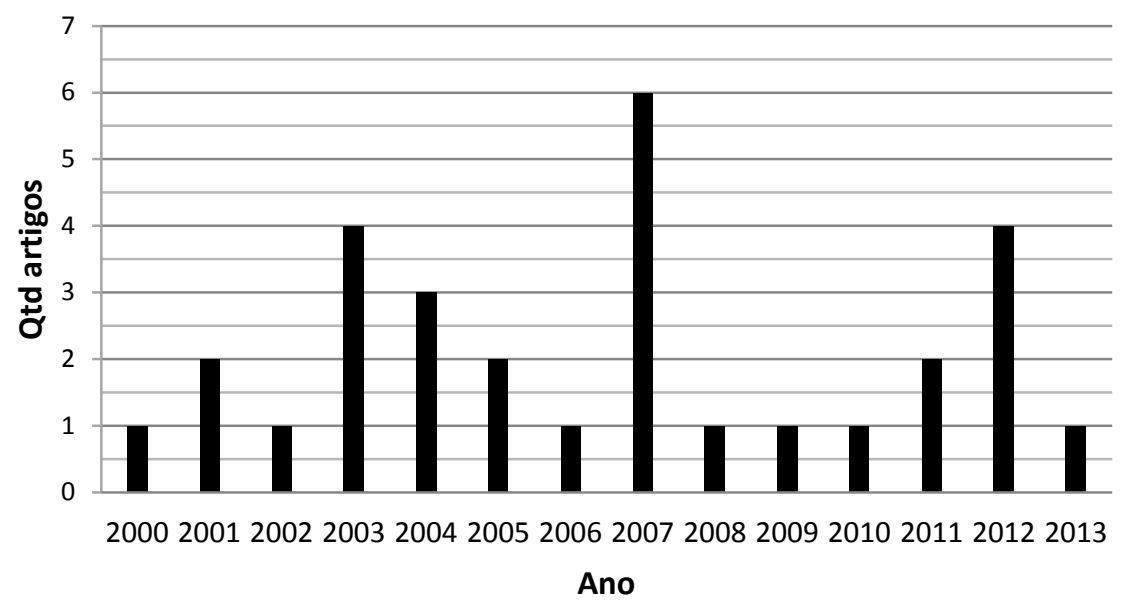

Figura 2

Quantidade de artigos publicados na área de eficácia escolar por ano no Brasil.

Quanto ao tipo de pesquisa, cinco artigos dentre os 30 selecionados são teóricos. Um deles discute a tradição de estudos quantitativos na área de efeito escola e o quanto esses estudos podem contribuir para compreensão das desigualdades de oportunidades (Koslinski \& Alves, 2012). Três deles abordam resultados das investigações brasileiras na área de eficácia escolar, destacam as variáveis utilizadas e as possibilidades de estudos futuros (Ferrão \& Fernandes, 2003; Franco \& Bonamino, 2005; Soares, 2007). Por fim, o último artigo teórico apresenta reflexões sobre aspectos da operacionalização de estudos 
sobre eficácia escolar, sobre os modelos de análise e os caminhos para apropriação dos resultados (Ferrão, 2012). Nenhum desses artigos realizou uma revisão sistemática da literatura brasileira.

Os estudos teóricos representam $16 \%$ dos artigos selecionados no levantamento bibliográfico. Esse percentual baixo parece indicar uma carência na sistematização e na reflexão dos achados na área, ações importantes para o desenvolvimento conceitual dos construtos investigados. Além disso, esses estudos são mais sintetizadores do que propositivos. Ressalta-se que uma das críticas à área de eficácia escolar é a existência de numerosos estudos empíricos, carentes de uma teoria sólida a ser verificada (Van Den Eeeden, Hox, \& Hauer, 1990).

Os 25 artigos restantes tratam de relatos de pesquisas. As informações gerais extraídas desses estudos empíricos estão apresentadas na Tabela 1. Nota-se que a maior parte dessas pesquisas foi realizada utilizando-se a base de dados do Sistema Nacional de Avaliação da Educação Básica (SAEB). Esse sistema de avaliação, desenvolvido pelo Governo Federal, tem como objetivo monitorar a qualidade, a equidade e a efetividade do sistema de educação básica. O SAEB ocorre de dois em dois anos e tem sido fonte de relevantes informações sobre o desempenho dos alunos em matemática e língua portuguesa e sobre características contextuais das escolas (Pestana, 1998).

Observa-se também na Tabela 1 que todas as pesquisas foram realizadas na educação básica e que a grande maioria utilizou a regressão multinível para análise dos dados. Essas duas características são condizentes com as pesquisas estrangeiras. A escolha pela educação básica é mais comum devido à existência de uma quantidade maior de informações, provindas de diversos sistemas avaliativos em larga escala, o que não é tão comum para a educação superior. Quanto ao uso de regressão multinível, essa é a técnica 
mais adequada, considerando a organização do sistema educacional em que alunos são agregados por escolas (Hox, 2010; Lee, 2008).

Tabela 1

Autores, ano de publicação, base de dados, etapa de ensino e tipo de análise dos relatos de pesquisa sobre eficácia escolar no Brasil.

\begin{tabular}{|c|c|c|c|c|}
\hline Autores & Ano & Base de dados & Série & Análise \\
\hline Barbosa \& Fernandes & 2000 & SAEB 1997 & $8^{\mathrm{a}} \mathrm{EF}$ & $\mathrm{RM}$ \\
\hline Soares, Alves, \& Oliveira & 2001 & UFMG 1998-2000 & $3^{\circ} \mathrm{EM}$ & $\mathrm{RM}$ \\
\hline Ferrão et al. & 2001 & SAEB 1999 & $4^{\mathrm{a}} \mathrm{EF}$ & RM \\
\hline Albanez, Ferreira, \& Franco & 2002 & SAEB 1999 & $8^{\mathrm{a}} \mathrm{EF}$ & RM \\
\hline Soares \& Alves & 2003 & SAEB 2001 & $8^{\mathrm{a}} \mathrm{EF}$ & $\mathrm{RM}$ \\
\hline Andrade, Franco, \& Carvalho & 2003 & SAEB 1999 & $3^{\circ} \mathrm{EM}$ & $\mathrm{RM}$ \\
\hline Soares, T. M. & 2003 & SIMAVE 2002 & $4^{\mathrm{a}} \mathrm{EF}$ & $\mathrm{RM}$ \\
\hline Soares (a) & 2004 & SAEB 2001 & $8^{\mathrm{a}} \mathrm{EF}$ & RM \\
\hline Soares (b) & 2004 & SAEB 2001 & $8^{\mathrm{a}} \mathrm{EF}$ & RM \\
\hline Jesus \& Laros & 2004 & SAEB 2001 & $8^{\mathrm{a}} \mathrm{EF}$ & $\mathrm{RM}$ \\
\hline Soares, T. M. & 2005 & SIMAVE 2002 & $4^{\mathrm{a}} \mathrm{EF}$ & RM \\
\hline Soares \& Andrade & 2006 & SIMAVE 2002/UFMG 2002-2004 & $3^{\circ} \mathrm{EM}$ & RM \\
\hline Franco et al. & 2007 & SAEB 2001 & $4^{\mathrm{a}} \mathrm{EF}$ & $\mathrm{RM}$ \\
\hline Andrade \& Laros & 2007 & SAEB 2001 & $3^{\circ} \mathrm{EM}$ & $\mathrm{RM}$ \\
\hline Nascimento & 2007 & Avaliação Estado da Bahia & $4^{\mathrm{a}}$ e $8^{\mathrm{a}} \mathrm{EF}$ & RL \\
\hline Alves \& Soares (a) & 2007 & Coleta própria & $5^{\mathrm{a}}$ e $6^{\mathrm{a}} \mathrm{EF}$ & $\mathrm{RM}$ \\
\hline Alves \& Soares (b) & 2007 & Coleta própria & $5^{\mathrm{a}} \mathrm{EF}$ & $\mathrm{RM}$ \\
\hline Alves \& Soares & 2008 & Coleta própria & $5^{\mathrm{a}}$ e $6^{\mathrm{a}} \mathrm{EF}$ & $\mathrm{RM}$ \\
\hline Teixeira & 2009 & Projeto GERES & Alfabetização & AQ \\
\hline Bonamino et al. & 2010 & PISA 2000 & 15 anos & RL \\
\hline Stocco \& Almeida & 2011 & Projetos GERES e NEPO & $1^{\mathrm{a}}-4^{\mathrm{a}} \mathrm{EF}$ & $\mathrm{AD}$ \\
\hline Rodrigues et al. & 2011 & SAEB 1997-2005 & $4^{\mathrm{a}} \mathrm{EF}$ & $\mathrm{RL} / \mathrm{AC}$ \\
\hline Silva, Bonamino, \& Ribeiro & 2012 & Coleta própria & EJA & AQ \\
\hline Laros, Marciano, \& Andrade & 2012 & SAEB 2001 & $3^{\circ} \mathrm{EM}$ & $\mathrm{RM}$ \\
\hline Ferrão \& Couto & 2013 & Projeto GERES & $1^{a}-4^{a} E F$ & RM \\
\hline
\end{tabular}

Notas: $\mathrm{EF}=$ Ensino Fundamental; $\mathrm{EM}=$ Ensino Médio; $\mathrm{AC}=$ Análise Contrafactual; $\mathrm{AD}=$ Análise Descritiva; $\mathrm{AQ}=$ Análise Qualitativa (Observações e Entrevistas); RM = Regressão Multinível; RL = Regressão Linear.

Na análise multinível, a dependência entre as observações pode ser avaliada pela correlação intraclasse (ICC), que representa a homogeneidade em um mesmo grupo e ao mesmo tempo a heterogeneidade entre grupos distintos (Laros \& Marciano, 2008). Quando a ICC é superior a $10 \%$ da variância total, recomenda-se a utilização de um modelo 
hierárquico (Lee, 2008). Nas pesquisas levantadas neste artigo, foram relatadas ICC entre 21 e $46 \%$ e, depois de corrigidas pelo contexto socioeconômico e cultural, entre 10 e $27 \%$.

No caso do Brasil, controlar as características socioeconômicas, sociais e culturais nos estudos educacionais faz-se essencial diante das desigualdades existentes no país e da seletividade de composição das escolas. Como mencionado por Fletcher (1998), "a composição da escola é fortemente condicionada pelas circunstâncias de seu contexto, especialmente a segregação residencial em seu entorno, provocada pelas diferenças socioeconômicas" (p.3). Esse controle é ainda mais necessário diante dos resultados que demonstram o grande impacto que o nível socioeconômico exerce sobre o desempenho escolar. Caso contrário, seria superestimado o efeito daquelas escolas que recebem alunos de maior poder aquisitivo e social.

Esses resultados estão em consonância com o estudo de Ferrão e Fernandes (2003) que indicou uma ICC geral em torno de $30 \%$ e em torno de 19\%, após controle contextual, no Brasil. Esses valores são bem superiores aos observados na maioria dos países desenvolvidos que giram em torno de $19 \%$ e, quando ajustado para o contexto, em torno de 10\% (Alves \& Soares, 2007b; Rutter \& Maughan, 2002). Contudo, estão bastante próximos dos valores encontrados nos estudos mexicanos que variam entre 12 a $43 \%$ (Carvallo-Pontón, 2010).

Dos 25 estudos empíricos, destaca-se que cinco possuem delineamento longitudinal. Esse tipo de delineamento é apontado como o mais adequado para a avaliação da eficácia escolar, sobretudo quando se trata de efeito-escola, uma vez que em estudos transversais a medida de desempenho representa o agregado de todo o aprendizado adquirido pelo estudante ao longo dos anos escolares e não o valor agregado pela escola cujos fatores associados estão sendo avaliados (Franco, Brooke, \& Alves, 2008). Dessa forma, surge um descompasso entre a medida de output e a medida das condições escolares. Entretanto, 
devido às dificuldades operacionais e políticas - tais como tempo, recursos financeiros, firmar e manter parcerias com instituições e manter o acompanhamento da amostra - os estudos longitudinais dentro e fora do Brasil ainda são em menor número.

Dos cinco estudos com delineamento longitudinal, três deles foram realizados utilizando-se uma base de dados composta por sete escolas de Belo Horizonte (Alves \& Soares, 2007a; Alves \& Soares, 2007b; Alves \& Soares, 2008). Os dois outros estudos utilizaram a base de dados do Projeto GERES (Ferrão \& Couto, 2013; Stocco \& Almeida, 2011). Esse Projeto surgiu da parceria entre seis centros universitários com tradição em avaliação da educação, a saber: o Laboratório de Avaliação da Educação da PUC-Rio (LAED), o Grupo de Avaliação e Medidas Educacionais da UFMG (GAME), o Laboratório de Observação e Estudos Descritivos da UNICAMP (LOED), a Linha de Pesquisa de Avaliação da Educação do Programa de Pós-Graduação em Educação da UFBA, o Centro de Avaliação da Educação da UFJF (CAEd) e a Universidade Estadual do Mato Grosso do Sul (UEMS). Trata-se de um estudo longitudinal de painel que teve início em 2005, no qual a mesma amostra de escolas e de alunos dos anos iniciais foi observada ao longo de quatro anos.

Ao avaliar o objetivo dos 25 artigos empíricos brasileiros na área de eficácia escolar, é possível categorizá-los em três segmentos: (1) artigos que buscavam estimar o efeitoescola ou o valor agregado pela escola; (2) artigos que buscavam avaliar o quanto a escola tem contribuído para a equidade e igualdade social; e (3) artigos que buscavam investigar os fatores contextuais e escolares relacionados à eficácia escolar.

Artigos relacionados ao efeito-escola.

Nesse primeiro segmento, encontram-se os cinco artigos de delineamento longitudinal e o artigo de Soares, Alves e Oliveira (2001). Neste último caso, para a estimação do efeito-escola, foi necessário adaptar uma medida indireta da habilidade 
acadêmica prévia a partir de informações do questionário acerca do histórico escolar. Essa adaptação foi necessária porque os estudos de efeito-escola buscam estimar o quanto a escola acrescentou ao aprendizado do aluno.

Dentre os artigos sobre efeito-escola, o estudo de Stocco e Almeida (2011) diferencia-se por ser o único a utilizar análise descritiva. Com base em dados do Projeto GERES e do Projeto de Estudo de Vulnerabilidade Sociodemográfica (NEPO - Unicamp), os autores discutiram a distribuição espacial das escolas e o desempenho escolar. Não foi encontrada uma recorrência clara em relação à disposição espacial das escolas quando relacionadas ao seu desempenho.

Os demais artigos utilizaram modelos multiníveis para estimação do efeito-escola. Em todos eles foi mencionada a importância de se realizar o controle da composição social da escola. Como já ressaltado, esse controle é necessário para que não seja atribuída à escola um efeito que advém de características sociais, culturais e econômicas dos alunos (Soares, Alves, \& Oliveira, 2001). Em geral, as variáveis utilizadas para o controle da composição social foram: nível socioeconômico do aluno, nível socioeconômico da escola (média do nível socioeconômico dos alunos), nível de escolaridade dos pais, sexo e etnia.

Em consonância com a literatura estrangeira, os resultados das pesquisas brasileiras também demonstram que a maior parte da variação entre resultados escolares pode ser explicada por fatores extraescolares. Contudo, mesmo após utilizar variáveis de controle da composição social, os estudos apontaram que a escola faz diferença no desempenho dos estudantes e efeitos diferenciados podem ser encontrados a depender da área de conhecimento (Alves \& Soares, 2007b).

Destaca-se ainda que, entre os artigos que investigaram o efeito-escola, três apresentaram uma abordagem quantitativa e qualitativa (mixed methods). Nos estudos de Alves e Soares (2007a), Alves e Soares (2007b), Alves e Soares (2008), após a 
identificação do efeito das escolas, esses resultados foram interpretados, utilizando-se dados das entrevistas realizadas com os professores.

\section{Artigos relacionados à equidade e igualdade.}

Nesse segundo segmento, encontram-se cinco artigos. Esses estudos corroboram a hipótese de que há no sistema educacional brasileiro desigualdades em termos de desempenho e de oportunidade de acesso a recursos. Vários estudos têm demonstrado, por exemplo, diferenças de desempenho por gênero, raça e nível socioeconômico. Compreender melhor essas diferenças é o foco dos artigos que tratam de igualdade e equidade. Ressalta-se, no entanto, que os artigos brasileiros focam principalmente aspectos em que a escola tem pouca interferência direta.

A produção científica no Brasil que avalia equidade e igualdade é pequena e bastante diversificada. Há um artigo que avalia diferenças em relação ao nível socioeconômico (Soares \& Andrade, 2006), um que avalia diferença entre raças (Soares \& Alves, 2003), um que avalia diferença entre gênero (Andrade, Franco, \& Carvalho, 2003) e dois que avaliam o quanto características escolares podem contribuir para a promoção da equidade (Soares, 2004a; Soares, 2004b).

O artigo de Soares e Alves (2003) mostra que existe grande diferença de desempenho entre alunos brancos e negros e que parte dessas diferenças deve-se ao efeito do nível socioeconômico. Quando verificados os efeitos moderadores de variáveis escolares, notouse que melhor qualificação docente, melhores salários e melhores equipamentos têm efeito significativo no sentido oposto ao desejado. Dessa forma, a melhora nas condições docentes e escolares tende a potencializar o efeito de raça sobre o desempenho, aumentando o hiato no desempenho escolar de alunos brancos e negros. 
Em outro artigo, Soares e Andrade (2006) verificaram o quanto as diferentes dependências administrativas do sistema escolar (federal, estadual, municipal e particular) têm atuado de modo a diminuir o efeito do nível socioeconômico sobre o desempenho. Os autores não encontraram efeito para as escolas estaduais, municipais e particulares. Já as escolas federais tenderam a aumentar o efeito do nível socioeconômico sobre o desempenho. Os autores apontaram ainda a inexistência de escolas com alta eficácia escolar e alta equidade.

De modo geral, os artigos sobre equidade têm demonstrado que os fatores produtores de melhora no desempenho acadêmico geram efeitos de intensidades diferentes entre os estudantes. Especificamente, alunos mais favorecidos tendem a usufruir mais das melhorias das condições escolares (Soares, 2004b). Esses resultados são perversos para o sistema educacional, pois demonstram um aumento das desigualdades na medida em que se melhora a eficácia escolar.

\section{Artigos relacionados aos fatores associados.}

Nesse terceiro segmento estão 14 artigos. O foco desses estudos é identificar políticas e práticas escolares que podem explicar o alto desempenho educacional dos estudantes. Dois desses artigos utilizaram uma abordagem qualitativa com uso de técnicas observacionais e de entrevistas: Teixeira (2009) e Silva, Bonamino e Ribeiro (2012). Esse tipo de abordagem é interessante na área de eficácia escolar, pois ela permite compreender mais profundamente determinados fenômenos e, dessa forma, corrobora para uma consolidação teórica.

No estudo de Teixeira (2009), tendo como base os resultados de estudos quantitativos que apontavam a importância do ambiente de aprendizado, do clima acadêmico e da infraestrutura, buscou-se avaliar os espaços destinados ao desenvolvimento 
de habilidades de leitura em seis escolas que apresentavam desempenhos diferenciados. Os resultados apontaram que os espaços da escola refletiam diferentes concepções de práticas pedagógicas e que nas escolas com melhores desempenhos era atribuída maior importância aos espaços e aos recursos escolares. Esse resultado mostra a relevância da execução de estudos qualitativos e quantitativos. Demonstra ainda o quanto alguns fatores apresentados na literatura são catalisadores ou inibidores de ambientes propícios ao desenvolvimento e, por isso, não podem ser interpretados de forma isolada (Ferrão, 2012).

Já no estudo de Silva, Bonamino e Ribeiro (2012), buscou-se analisar três escolas da rede municipal do Rio de Janeiro que integram o programa de educação de jovens e adultos e que têm apresentado bons resultados. Os autores concluem que muitos dos fatores associados ao desempenho encontrados nos estudos relativos à educação regular são também válidos para a educação de jovens e adultos.

Dentre os estudos quantitativos, a abordagem econométrica utilizada por Rodrigues, Rios-Neto e Pinto (2011) se diferencia das demais. Os autores buscaram avaliar o impacto do nível socioeconômico no desempenho escolar avaliado pelo SAEB entre 1997 e 2005. Para tanto, os autores utilizaram um método de decomposição contrafactual que permite isolar a contribuição da variação na composição e retorno do nível socioeconômico sobre a variação na média e distribuição do desempenho escolar. Os resultados confirmam os achados da literatura que apontam o forte efeito do nível socioeconômico sobre o desempenho. Além disso, os resultados indicaram que a queda no desempenho em matemática no SAEB pode ser explicada pela democratização das oportunidades de acesso à escola e consequente queda no nível socioeconômico geral.

Com relação aos demais 11 estudos quantitativos, eles utilizaram em suas análises regressão linear simples ou regressão multinível (Albanez, Ferreira, \& Franco, 2002; Andrade \& Laros, 2007; Barbosa \& Fernandes, 2000; Bonamino, Alves, Franco, \& Cazelli, 
2010; Ferrão et al., 2001; Franco et al., 2007; Jesus \& Laros, 2004; Laros, Marciano, \& Andrade, 2012; Nascimento, 2007; Soares, 2003; Soares, 2005). A fim de melhor sumarizar os achados, as variáveis investigadas em cada estudo foram categorizadas com base no modelo integrado de Scheerens (1990): contexto, input, processo e output. As Tabelas 2, 3 e 4 apresentam os resultados dessa sumarização. Nas Tabelas, foram utilizados os sinais $\uparrow, \downarrow,-$, para representar uma relação positiva, negativa ou não significativa com o desempenho, respectivamente. A quantidade de vezes que os sinais aparecem representa a quantidade de artigos que utilizaram a variável/fator.

Tabela 2

Fatores/Variáveis contextuais utilizados nos estudos brasileiros de eficácia escolar e sua relação com desempenho.

\begin{tabular}{|c|c|c|c|c|c|c|c|c|c|c|c|}
\hline Fatores/Variáveis de Contexto & 1 & 2 & 3 & 4 & 5 & 6 & 7 & 8 & 9 & 10 & 11 \\
\hline Nível socioeconômico do aluno & $\downarrow$ & $\downarrow$ & $\downarrow$ & - & - & $\uparrow$ & $\uparrow$ & $\uparrow$ & $\uparrow$ & $\uparrow$ & \\
\hline Nível socioeconômico da turma/escola & $\uparrow$ & $\uparrow$ & $\uparrow$ & $\uparrow$ & $\uparrow$ & $\uparrow$ & $\uparrow$ & $\uparrow$ & $\uparrow$ & & \\
\hline Sexo (masculino) & $\uparrow$ & $\uparrow$ & $\uparrow$ & $\uparrow$ & $\downarrow$ & $\downarrow$ & & & & & \\
\hline Etnia (não branco) & $\downarrow$ & $\downarrow$ & $\downarrow$ & $\downarrow$ & $\downarrow$ & $\downarrow$ & $\downarrow$ & & & & \\
\hline Etnia agregada (não branco) & $\downarrow$ & $\downarrow$ & - & & & & & & & & \\
\hline Escolaridade do pai ou da mãe & $\uparrow$ & $\uparrow$ & $\uparrow$ & - & & & & & & & \\
\hline Diálogo familiar & $\uparrow$ & & & & & & & & & & \\
\hline Status ocupacional dos pais & $\uparrow$ & & & & & & & & & & \\
\hline Recursos educacionais familiares & $\uparrow$ & & & & & & & & & & \\
\hline Disponibilidade de recursos culturais (livros) em casa & $\uparrow$ & $\uparrow$ & & & & & & & & & \\
\hline Disponibilidade de recursos culturais em casa agregado & $\uparrow$ & & & & & & & & & & \\
\hline Grau de urbanização do município & - & & & & & & & & & & \\
\hline Rendimento médio do município & - & & & & & & & & & & \\
\hline Garantia do mínimo constitucional na educação & - & & & & & & & & & & \\
\hline Gastos efetivos em educação no município & - & & & & & & & & & & \\
\hline Relação $n^{\circ}$ de docentes $/ n^{\circ}$ de alunos & - & & & & & & & & & & \\
\hline Rede (particular) & $\uparrow$ & $\uparrow$ & $\uparrow$ & & & & & & & & \\
\hline
\end{tabular}

Dentre as variáveis de contexto, confirma-se a relação consistente e positiva do nível socioeconômico da escola sobre o desempenho do aluno já encontrada em estudos estrangeiros (Coleman et al., 1966; Fletcher, 1998). Quanto ao nível socioeconômico do aluno, a sua relação com o desempenho parece não ser tão clara. Ocorre que, quando o nível socioeconômico do aluno é inserido em conjunto com o nível socioeconômico da 
escola, o efeito da primeira variável tende a ser bastante pequeno e muitas vezes negativo, isso porque essas variáveis apresentam-se altamente correlacionadas.

Outra variável que aponta relação consistente com desempenho é raça. Os resultados demonstram que alunos negros apresentam, em média, desempenho inferior ao dos alunos brancos. Essa relação mostra a desigualdade presente no sistema educacional brasileiro. Ferrão et al. (2001) já indicava no início dos anos 2000 a necessidade e importância de se procurar explicação para o efeito de raça sobre o desempenho. Todavia, pouco se avançou sobre a compreensão desse efeito.

Quanto à variável sexo, apesar de parecer que os resultados são controversos, eles não são. Os diferentes sentidos (efeitos positivos e negativos) estão relacionados com a disciplina avaliada. Os estudos apontam que quando o desempenho avaliado é em matemática, os meninos têm resultados melhores (Albanez, Ferreira, \& Franco, 2002; Andrade \& Laros, 2007; Barbosa \& Fernandes, 2000; Franco et al., 2007). Quando o desempenho avaliado é em língua portuguesa, as meninas têm resultados melhores (Soares, 2003; Soares, 2005).

Vale destacar que a escolaridade dos pais, apesar de aparecer com efeito positivo em somente três estudos, em geral tem apresentado uma relação forte e consistente, sobretudo quando se trata da escolaridade da mãe. Ocorre que em muitos estudos essa variável é agregada na composição do fator de nível socioeconômico e, por isso, ela não é incluída isoladamente no modelo. Para as demais variáveis ainda é arriscado afirmar alguma tendência.

Ao avaliar a Tabela 3, ressalta-se o efeito negativo do atraso escolar sobre o desempenho nos oito estudos em que essa variável foi avaliada. Esse resultado aponta que, quando o estudante é reprovado em algum momento, ele tende a ter um desempenho 
inferior aos demais estudantes no decorrer da trajetória escolar, mesmo ele tendo cursado algumas vezes uma determinada série.

Tabela 3

Fatores/Variáveis de input utilizados nos estudos brasileiros de eficácia escolar e sua relação com desempenho.

\begin{tabular}{|c|c|c|c|c|c|c|c|c|c|c|c|}
\hline Fatores/Variáveis de Input & 1 & 2 & 3 & 4 & 5 & 6 & 7 & 8 & 9 & 10 & 11 \\
\hline Número de alunos na escola & - & & & & & & & & & & \\
\hline Aluno frequentou a pré-escola & $\uparrow$ & & & & & & & & & & \\
\hline Atraso escolar/defasagem idade-série & $\downarrow$ & $\downarrow$ & $\downarrow$ & $\downarrow$ & $\downarrow$ & $\downarrow$ & $\downarrow$ & $\downarrow$ & & & \\
\hline Bolsa Escola & $\downarrow$ & & & & & & & & & & \\
\hline Aluno trabalha & $\downarrow$ & $\downarrow$ & $\downarrow$ & & & & & & & & \\
\hline Gosta da disciplina & $\uparrow$ & $\uparrow$ & $\uparrow$ & & & & & & & & \\
\hline Abandono escolar & $\downarrow$ & & & & & & & & & & \\
\hline Insuficiência de recursos financeiros & $\downarrow$ & & & & & & & & & & \\
\hline Turno & - & & & & & & & & & & \\
\hline Atraso escolar/defasagem idade-série agregado & $\downarrow$ & $\downarrow$ & $\downarrow$ & $\downarrow$ & & & & & & & \\
\hline Gosta da disciplina agregado & - & & & & & & & & & & \\
\hline Aluno trabalha agregado & $\downarrow$ & $\downarrow$ & $\downarrow$ & & & & & & & & \\
\hline Estado de conservação dos equipamentos & $\uparrow$ & $\uparrow$ & $\uparrow$ & $\uparrow$ & & & & & & & \\
\hline Infraestrutura escolar & $\uparrow$ & $\uparrow$ & $\uparrow$ & $\uparrow$ & & & & & & & \\
\hline Formação professor & $\uparrow$ & $\uparrow$ & $\uparrow$ & & & & & & & & \\
\hline Existência de pessoal e recursos & $\uparrow$ & - & & & & & & & & & \\
\hline Idade média dos professores & $\downarrow$ & & & & & & & & & & \\
\hline
\end{tabular}

Outra informação relevante apresentada na Tabela 3 é a influência positiva que a infraestrutura e o bom estado de conservação dos equipamentos escolares exercem sobre o desempenho. Essa informação diverge dos resultados encontrados na literatura estrangeira (Albanez, Ferreira, \& Franco, 2002). Isso decorre da precariedade do sistema educacional brasileiro que ainda não garante uma estrutura mínima para o adequado funcionamento da escola. Em países desenvolvidos a variância nos quesitos referentes à infraestrutura e equipamentos é tão pequena que não é significativa para explicar as diferenças no desempenho acadêmico (Murillo \& Roman, 2011).

Quanto às variáveis processuais (Tabela 4), nota-se uma carência de informações que nos permita afirmar quais variáveis da escola podem realmente fazer a diferença no 
desenvolvimento acadêmico dos estudantes. Os resultados mais consistentes são relativos a passar/fazer dever de casa e ao bom clima escolar.

Considerando os fatores processuais que contribuem para a constituição de escolas eficazes levantados na revisão de literatura de Sammons, Hillman e Mortimore (1995), as três variáveis correspondem aos fatores: ambiente de aprendizagem e ensino e objetivos claros. A diferença é que os fatores utilizados na literatura internacional são mais abrangentes. Por exemplo, o fator ensino e objetivos claros envolve aspectos como clareza de propósitos, aulas bem estruturadas, ensino adaptável, entre outros que estão além do simples passar dever de casa.

Tabela 4

Fatores/Variáveis de processo utilizados nos estudos brasileiros de eficácia escolar e sua relação com desempenho.

\begin{tabular}{|c|c|c|c|c|c|c|c|c|c|c|c|}
\hline Fatores/Variáveis de Processo & 1 & 2 & 3 & 4 & 5 & 6 & 7 & 8 & 9 & 10 & 11 \\
\hline Fazer dever de casa & $\uparrow$ & $\uparrow$ & $\uparrow$ & $\uparrow$ & & & & & & & \\
\hline Uso do computador para fazer dever de casa & $\downarrow$ & $\downarrow$ & & & & & & & & & \\
\hline Faltas aluno & $\downarrow$ & & & & & & & & & & \\
\hline Faltas aluno agregado & $\downarrow$ & & & & & & & & & & \\
\hline Faltas professor & $\downarrow$ & $\downarrow$ & & & & & & & & & \\
\hline Passar dever de casa & $\uparrow$ & $\uparrow$ & $\uparrow$ & $\uparrow$ & $\uparrow$ & & & & & & \\
\hline Bom clima escolar & $\uparrow$ & $\uparrow$ & $\uparrow$ & $\uparrow$ & - & & & & & & \\
\hline Cobrança/incentivo dos pais & $\downarrow$ & $\downarrow$ & $\downarrow$ & & & & & & & & \\
\hline Ênfase em resolução de problemas & $\uparrow$ & & & & & & & & & & \\
\hline Liderança e trabalhos colaborativos & $\uparrow$ & $\uparrow$ & - & & & & & & & & \\
\hline Interesse e comprometimento do professor & $\uparrow$ & $\uparrow$ & $\uparrow$ & & & & & & & & \\
\hline Taxa de estudo & $\uparrow$ & & & & & & & & & & \\
\hline Comparação com outros colegas & $\uparrow$ & & & & & & & & & & \\
\hline Sistema de ensino em ciclos & $\downarrow$ & & & & & & & & & & \\
\hline Cobrança/incentivo dos pais agregado & $\uparrow$ & $\downarrow$ & & & & & & & & & \\
\hline Programas de recuperação de notas & - & & & & & & & & & & \\
\hline Professor exigente em sala de aula & $\uparrow$ & $\uparrow$ & & & & & & & & & \\
\hline Expectativa do professor & $\uparrow$ & & & & & & & & & & \\
\hline
\end{tabular}

De modo geral é possível encontrar certa correspondência entre as variáveis/fatores encontradas na literatura brasileira (Tabela 4) e os 11 fatores sumarizados pela revisão de literatura de Sammons, Hillman e Mortimore (1995), a saber: (1) liderança profissional; (2) 
objetivos e visões compartilhadas; (3) um ambiente de aprendizagem; (4) concentração no ensino e na aprendizagem; (5) ensino e objetivos claros; (6) altas expectativas; (7) incentivo positivo; (8) monitoramento do progresso; (9) direitos e responsabilidades dos alunos; (10) parceria casa-escola e (11) uma organização orientada à aprendizagem. Todavia, há a necessidade de se investir na construção de medidas mais adequadas para avaliar as características processuais das escolas e de se realizar mais estudos para se testar a fidedignidade dos resultados.

Por fim, em relação ao critério de output utilizado nos estudos quantitativos, três estudos utilizaram o desempenho em matemática, cinco em língua portuguesa, um realizou as análises para matemática e língua portuguesa em separado e dois consideraram no modelo o desempenho em diversas disciplinas. Destaca-se que todos os estudos quantitativos utilizaram como critério uma medida acadêmica. Para Soares e Alves (2003) isso não significa privilegiar o domínio cognitivo em relação aos demais domínios que devem ser desenvolvidos na escola, mas sim de reconhecer a importância das competências cognitivas para se atingir outros objetivos, além desse domínio ser bastante dependente da estrutura escolar. Acrescenta-se ainda a ausência de medidas sistemáticas referentes aos outros domínios, o que dificulta a realização de pesquisas.

\section{Conclusões e direções para pesquisas futuras}

A vasta produção na área de eficácia escolar tem apontado para um conjunto de fatores (contextuais, organizacionais, de monitoramento e pedagógicos) que tornam a escola eficaz, ao contribuir com o desenvolvimento do estudante, feita a ressalva de que não se pode esperar que ela elimine completamente as inequidades sociais e biológicas (Rutter \& Maughan, 2002). De modo geral, os resultados das pesquisas brasileiras 
convergem para os resultados observados em outros países. No entanto, fatores como infraestrutura e estado de conservação dos equipamentos ainda fazem diferença sobre o desempenho acadêmico no Brasil (Albanez, Ferreira, \& Franco, 2002; Andrade \& Laros, 2007; Ferrão et al., 2001; Franco et al., 2007; Jesus \& Laros, 2004). Nesse contexto, na ausência de elementos tão básicos, torna-se difícil identificar efeitos homogêneos e significativos referentes a fatores processuais como práticas pedagógicas.

Para Murillo e Román (2011), esses resultados demonstram a necessidade de ajuste dos modelos de eficácia escolar para populações mais precárias ou países em desenvolvimento, para que isso não culmine na aplicação de políticas inadequadas destinadas a países em outro patamar de desenvolvimento educacional. Portanto, além de ainda permanecer em aberto a questão de como promover mudanças apropriadas naquelas escolas que não estão funcionando adequadamente, modelos teóricos específicos para países em desenvolvimento com problemas de equidade precisam ser desenvolvidos.

A Oficina Regional de Educação para a América Latina e Caribe (OREALC/UNESCO) tem investido no desenvolvimento de ações voltadas para a produção de informação contextualizada sobre a aprendizagem dos alunos e a análise dos fatores associados ao desempenho, por exemplo, com as aplicações de três estudos comparativos (PERCE, SERCE e TERCE). Murillo (2008) destaca que Chile, México, Colômbia, Argentina e Brasil são os países com mais pesquisas na área de eficácia escolar e reforça que é preciso investir na sua continuidade.

Nota-se uma tendência em tornar a área mais teoricamente dirigida, com a proposição de modelos que integrem várias perspectivas de pesquisa (insumos, eficácia escolar, eficácia docente, equidade, igualdade, avaliação de sistemas) de modo a realizar uma análise mais global e de interação entre as variáveis (Scheerens, 2000). A análise dessa interação, em conjunto com a análise de aspectos como consistência, coesão, 
constância e controle, propostos, por exemplo, no modelo dinâmico, pode ser um dos caminhos para melhor compreender quais mudanças são mais efetivas (Kyriakides, 2008).

É preciso mencionar que parte da dificuldade em se validar modelos e desenvolver uma fundamentação teórica da área está na construção das medidas, sobretudo aquelas referentes aos aspectos processuais da escola. Faltam consistência e definições claras dessas medidas (Kyriakides, 2008). Por exemplo, construtos bastante complexos como práticas pedagógicas muitas vezes se reduzem a uma variável "fazer dever de casa". Certamente, parte do problema de medida está igualmente associado à ausência de informações nos questionários das avaliações em larga escala, os quais devem ser aprimorados (Karino, Vinha, \& Laros, 2014).

A partir da revisão bibliográfica fica claro também que, entre os desafios da área de eficácia escolar, está o delineamento e execução de pesquisas longitudinais (Reynolds et al., 2011). A estimação do efeito-escola é mais adequada por meio de um delineamento em que os mesmos estudantes são acompanhados ao longo do tempo. Porém, diante da dificuldade de condução de estudos longitudinais, é preciso investir, em paralelo, em outras estratégias de análise que possibilitem uma melhor estimação tanto do efeito-escola quanto das variáveis que contribuem para a promoção da eficácia escolar.

Felizmente, uma das tendências que parece surgir nos artigos sobre eficácia escolar é a realização de estudos de abordagem quanti e quali ou estudos multimétodo. Os estudos quantitativos são subsidiários de informações que permitem identificar sistemas modelo e fatores que parecem fazer a diferença. Tais resultados podem melhor orientar estudos com abordagem qualitativa, que permitem uma visão mais analítica de como as escolas funcionam e, consequentemente, podem contribuir para melhor compreensão do fenômeno. Assim, estimula-se tanto quanto possível a realização de estudos com abordagem multimétodo e a parceria de estudos quanti e quali. 
Outro desafio da área é encontrar respostas para a promoção de escolas mais eficazes e mais equânimes. Os resultados estrangeiros e brasileiros têm consistentemente apontado que há desigualdade entre estudantes por nível socioeconômico, raça e gênero. Mais desanimador ainda são os resultados dos estudos sobre equidade que mostram que, ao se promover eficácia escolar, se promove um aumento das desigualdades, uma vez que estudantes mais favorecidos tendem a usufruir mais das melhorias no ambiente escolar. Debruçar-se sobre a problemática de como alcançar um sistema educacional mais igual e equânime é um desafio urgente.

Mesmo diante de grandes avanços ocorridos na área de avaliação educacional e nos estudos sobre eficácia escolar, há ainda muitas perguntas não respondidas. Buscou-se, a partir da análise das publicações brasileiras em artigos científicos e da confrontação com os resultados da literatura estrangeira, demonstrar necessidades, limitações e lacunas da área de eficácia escolar, bem como indicar caminhos para o crescimento e aprimoramento da produção científica. Espera-se, assim, que essa sumarização e reflexão sobre os achados instiguem pesquisadores e provoquem uma nova agenda de pesquisa.

\section{Referências}

Albanez, A., Ferreira, F., \& Franco, F. (2002). Qualidade e equidade no ensino fundamental brasileiro. Pesquisa e Planejamento Econômico, 32 (3), 453-475.

Alves, M. T. G., \& Soares, J. F. (2007a). As pesquisas sobre os efeitos das escolas: Contribuições metodológicas para a sociologia da educação. Sociedade e Estado, 22(2), 435-473.

Alves, M. T. G., \& Soares, J. F. (2007b). Efeito-escola e estratificação escolar: O impacto da composição de turmas por nível de habilidade dos alunos. Educação em Revista, $45,25-58$.

Alves, M. T. G., \& Soares, J. F. (2008). O efeito das escolas no aprendizado dos alunos: Um estudo com dados longitudinais do Ensino Fundamental. Educação e Pesquisa, 34(3), 527-544. 
Andrade, J. M., \& Laros, J. A. (2007). Fatores associados ao desempenho escolar: Um estudo multinível com os dados do SAEB/2001. Psicologia: Teoria e Pesquisa, 23, $33-42$.

Andrade, M., Franco, C., \& Carvalho, J. B. P. (2003). Gênero e desempenho em matemática ao final do ensino médio: Quais as relações? Estudos em Avaliação Educacional, 27, 77-95.

Barbosa, M. E., \& Fernandes, C. (2000). Modelo multinível: Uma aplicação a dados de avaliação educacional. Estudos em Avaliação Educacional, 22, 135-153.

Bonamino, A., Alves, F., Franco, C., \& Cazelli, S. (2010). Os efeitos das diferentes formas de capital no desempenho escolar: Um estudo à luz de Bourdieu e de Coleman. Revista Brasileira de Educação, 15(45), 487-594.

Carvallo-Pontón, M. (2010). Eficacia escolar: Antecedentes, hallazgos y futuro. Revista Internacional de Investigación en Educación, 3, 199-214.

Coleman, J. S., Campbell, E., Hobson, C., McPartland, J., Mood, A., Weinfeld, R., \& York, R. (1966). Equality of educational opportunity. Washington, DC: US Department of Health, Education \& Welfare.

Edmonds, R. (1979). Effective schools for the urban poor. Educational Leadership, 37, 1527.

Ferrão, M. E. (2012). Avaliação educacional e modelos de valor acrescentado: Tópicos de reflexão. Educação \& Sociedade, 33(119), 455-469.

Ferrão, M. E., \& Couto, A. (2013). Indicador de valor acrescentado e tópicos sobre a consistência e estabilidade: Uma aplicação ao Brasil. Ensaio: Avaliação de Políticas Públicas Educacionais, 21(78), 131-164.

Ferrão, M. E., \& Fernandes, C. O. (2003). O efeito-escola e a mudança: Dá para mudar? Evidências da investigação brasileira. Revista Electronica Iberoamericana sobre Calidad, Eficácia y Cambio en Educación, 1(1), 1-13.

Ferrão, M. E., Beltrão, K. I., Fernandes, C., Santos, D., Suarez, M., \& Andrade, A. do C. (2001). O SAEB - Sistema Nacional de Avaliação da Educação Básica: Objetivos, características e contribuições na investigação da escola eficaz. Revista Brasileira de Estudos de População, 18(1/2), 111-130.

Fletcher, P. R. (1998). À procura do ensino eficaz. Relatório técnico. Brasília: MEC/DAEB.

Franco, C., \& Bonamino, A. (2005). A pesquisa sobre característica de escolas eficazes no Brasil: Breve revisão dos principais achados e alguns problemas em aberto. Educação on-line: Revista do Programa de Pós-graduação em Educação, 1, 1-13. 
Franco, C., Brooke, N., \& Alves, F. (2008). Estudo longitudinal sobre qualidade e equidade no ensino fundamental brasileiro: GERES 2005. Ensaio: Avaliação de Políticas Públicas Educacionais, 16(61), 625-638.

Franco, C., Ortigão, I., Albernaz, A., Bonamino, A., Aguiar, G., Alves, F., \& Sátyro, N. (2007). Qualidade e equidade em educação: Reconsiderando o significado de "fatores intraescolares". Ensaio: Avaliação de Políticas Públicas Educacionais, 15(55), $277-$ 298.

Goldstein, H. (2010). Multilevel statistical models. (4th edition). London: Wiley.

Hox, J. (2010). Multilevel analysis: Techniques and applications. Mahwah, New Jersey: Lawrence Erlbaum Associates.

Jesus, G. R., \& Laros, J. A. (2004). Eficácia escolar: Regressão multinível com dados de avaliação em larga escala. Avaliação Psicológica, 3, 93-106.

Karino, C. A., Vinha, L. G. do A., \& Laros, J. A. (2014). Os questionários do SAEB: O que eles realmente medem? Estudos em Avaliação Educacional, 25(59), 270-297.

Koslinski, M. C., \& Alves, F. (2012). Novos olhares para as desigualdades de oportunidades educacionais: A segregação residencial e a relação favela-asfalto no contexto carioca. Educação \& Sociedade, 33(120), 805-831.

Kyriakides, L. (2008). Testing the validity of the comprehensive model of educational effectiveness: A step towards the development of a dynamics model of effectiveness. School Effectiveness and School Improvement, 19(4), 429-446.

Laros, J. A., \& Marciano, J. L. (2008). Análise multinível aplicada aos dados do NELS 88. Estudos em Avaliação Educacional, 19(40), 263-278.

Laros, J. A., Marciano, J. L., \& Andrade, J. M. de (2012). Fatores associados ao desempenho escolar em Português: Um estudo multinível por regiões. Ensaio: Avaliação de Políticas Públicas Educacionais, 20(77), 623-646.

Lee, V. L. (2008). Utilização de modelos lineares hierárquicos lineares para estudar contextos sociais: O caso dos efeitos da escola. In N. Brooke \& J. F. Soares (Eds.), Pesquisa em eficácia escolar: Origem e trajetórias (pp. 273-296). Belo Horizonte: Editora UFMG.

Mortimore, P. (1991). The nature and findings of school effectiveness research in primary sector. In S. Ridell \& E. Peck (Eds.), School effectiveness research: Its message for school improvement (pp. 9-19). London: HMSO.

Mortimore, P., Sammons, P., Stoll, L., Lewis, D. \& Ecob, R. (1988). School matters: The junior years. Shepton Mallett: Open Books.

Murillo, F. J. (2008). Enfoque, situación y desafios de la investigación sobre eficacia escolar em América Latina y el Caribe. In UNESCO (Ed.), Eficacia escolar y factores asociados en América Latina y el Caribe (pp. 7-47). Santiago, Chile: UNESCO. 
Murillo, F. J., \& Román, M. (2011). School infrastructure and resources do matter: Analysis of the incidence of school resources on the performance of Latin American students. School Effectiveness and School Improvement, 22(1), 29-50.

Nascimento, P. A. M. M. (2007). Desempenho escolar e gastos municipais por aluno em educação: Relação observada em municípios baianos para o ano 2000. Ensaio: Avaliação de Políticas Públicas Educacionais, 15(56), 393-412.

Odden, A. (1982). Schools can improve: Local strategies need state backing. State Education Leader, 1(3), 1-3.

Pestana, M. I. O. (1998). Sistema de avaliação brasileiro. Revista brasileira de estudos pedagógicos, 79(191), 65-73.

Reynolds, D., Sammons, P., Fraine, B. D., Townsend, T., \& Damme, J. V. (2011). Educational effectiveness research (EER): A state of the art review. Trabalho apresentado no International Congress for School Effectiveness and Improvement, Cyprus.

Reynolds, D., Teddlie, C., Creemers, B., Scheerens, J., \& Townsend, T. (2000). An introduction to school effectiveness research. In C. Teddlie e D. Reynolds (Eds.), The international handbook of school effectiveness research (pp. 3-25). New York: Routledge.

Rodrigues, C. G., Rios-Neto, E. L. G., \& Pinto, C. C. de X. (2011). Diferenças intertemporais na média e distribuição do desempeno escolar no Brasil: O papel do nível socioeconômico, 1997 a 2005. Revista Brasileira de Estudos Populacionais, 28(1), 5-36.

Rutter, M., \& Maughan, B. (2002). School effectiveness findings 1979-2002. Journal of School Psychology, 40(6), 451-475.

Rutter, M., Maughan, B., Mortimore, P., Ouston, J., \& Smith, A. (1979). Conclusões, especulações e implicações. In N. Brooke e J. F. Soares (Eds.), Pesquisa em eficácia escolar: Origem e trajetórias (pp. 225-251). Belo Horizonte: Editora UFMG.

Sammons, P., Hillman, J., \& Mortimore, P. (1995). Key characteristics of effective schools: A review of school effectiveness research. London: Office for Standards in Education [OFSTED].

Scheerens, J. (1990). School effectiveness and the development of process indicators of school functioning. In J. Scheerens (Ed.), School effectiveness and school improvement (pp. 61-80). Lisse: Swets and Zeitlinger.

Scheerens, J. (2000). Improving school effectiveness (Fundamentals of Educational Planning $\mathrm{N}^{\circ}$. 68). Paris: UNESCO/International Institute for Educational Planning.

Silva, J., Bonamino, A. M. C. de, \& Ribeiro, V. M. (2012). Escolas eficazes na educação de jovens e adultos: Estudo de casos na rede municipal do Rio de Janeiro. Educação em Revista, 28(2), 367-392. 
Soares, J. F. (2004a). Qualidade e equidade na educação brasileira: A evidência do SAEB2001. Archivos Analíticos de Políticas Educativas, 12(38), 1-28.

Soares, J. F. (2004b). O efeito da escola no desempenho cognitivo de seus alunos. Revista Electronica Iberoamericana sobre Calidad, Eficácia y Cambio en Educación, 2(2), 83-104.

Soares, J. F. (2007). Melhoria do desempenho cognitivo dos alunos no ensino fundamental. Cadernos de Pesquisa, 37(130), 135-160.

Soares, J. F., \& Alves, M. T. G. (2003). Desigualdades raciais no sistema brasileiro de educação básica. Educação e Pesquisa, 29(1), 147-165.

Soares, J. F., \& Andrade, R. J. de (2006) Nível socioeconômico, qualidade e equidade das escolas de Belo Horizonte. Ensaio: Avaliação de Políticas Públicas Educacionais, 14(50), 107-126.

Soares, J. F., Alves, M. T. G., \& Oliveira, R. M. (2001). O efeito de 248 escolas de nível médio no vestibular da UFMG nos anos de 1998, 1999 e 2000. Estudos em Avaliação Educacional, 24, 69-117.

Soares, T. M. (2003). Influência do professor e do ambiente em sala de aula sobre a proficiência alcançada pelos alunos avaliados no SIMAVE 2002. Estudos em Avaliação Educacional, 28, 103-124.

Soares, T. M. (2005). Modelo de 3 níveis hierárquicos para a proficiência dos alunos de 4a série avaliados no teste de língua portuguesa do SIMAVE/PROEB 2002. Revista Brasileira de Educação, 29, 73-88.

Stocco, S., \& Almeida, L. C. (2011). Escolas municipais de Campinas e vulnerabilidade sociodemográfica: Primeiras aproximações. Revista Brasileira de Educação, 16(48), 663-814.

Teixeira, R. A. (2009). Espaços, recursos escolares e habilidades de leitura de estudantes da rede pública municipal do Rio de Janeiro: Estudo exploratório. Revista Brasileira de Educação, 14(41), 232-390.

Teodorovic, J. (2009). School Effectiveness: Literature review. Зборник Института за педагошка истраживања (Serbian Institute for Educational Research), 41, 297-314.

Van den Eeden, P., Hox, J., \& Hauer, J. (1990). Theory and model in multilevel research: Convergence or divergence? Amsterdam: SISWO.

Willms, J. D. (1992). Monitoring school performance. Washington, DC: The Falmer Press.

Willms, J. D., \& Raudenbush, S. W. (1989). A longitudinal hierarchical linear model for estimating school effects and their stability. Journal of Educational Measurement, 26, 209-232. 


\title{
Manuscrito 2
}

\section{PILLARS OF GOOD EDUCATION: ANALYZING}

THE BRAZILIAN SCHOOL SYSTEM

\begin{abstract}
There are some elements that every educational system should have. These elements are the guarantee of a minimum quality and equality of education provided by the government. From this perspective, the Educational Prosperity model, based on a large body of empirical results in Educational Effectiveness Research, defines the school pillars which are the core of the learning process and the success of the education system. In the attempt to analyze the Brazilian school system, the goals of this study are to build measures for the pillars of Educational Prosperity model and to evaluate the access to these pillars in the Brazilian context. The study was conducted by using the database of a Brazilian national assessment, named Prova Brasil, applied in 2009. Using the questionnaires of the assessment, it was possible to construct measures for all the pillars defined by the model. Nevertheless, some pillars were not completely addressed. Considering the measures, we note that the Brazilian educational system has equity problems and has many aspects to improve. This study makes the educational situation in Brazil even more evident and also allows better comparability with other educational systems.
\end{abstract}

Keywords: quality of education; development measures; educational prosperity. 


\section{Resumo}

Há alguns elementos que todo sistema de ensino deve ter. Estes elementos são a garantia da qualidade mínima e da igualdade da educação fornecida pelo governo. A partir desta perspectiva, o modelo de Prosperidade Educacional, com base em um grande conjunto de resultados empíricos das pesquisas de Eficácia Escolar, define os pilares da escola que são o núcleo do processo de aprendizagem e do sucesso do sistema de ensino. Tentando analisar o sistema escolar brasileiro, os objetivos deste estudo são construir medidas para os pilares do modelo de Prosperidade Educacional e avaliar o acesso a esses pilares no contexto brasileiro. O estudo foi realizado utilizando o banco de dados de uma avaliação nacional brasileira, denominada Prova Brasil, aplicada em 2009. Usando os questionários da avaliação, foi possível construir medidas para todos os pilares definidos pelo modelo. No entanto, alguns pilares não foram completamente contemplados. Considerando as medidas, podemos constatar que o sistema educacional brasileiro tem problemas de equidade e tem muitos aspectos a melhorar. Este estudo torna a situação educacional no Brasil ainda mais evidente e também permite uma melhor comparabilidade com outros sistemas de ensino.

Palavras-chave: qualidade da educação; desenvolvimento de medidas; prosperidade educacional. 
Education is a fundamental right provided in the Universal Declaration of Human Rights, essential for the exercise of all other human rights and with recognized importance for the empowerment promotion (United Nations, 1948). The assurance of this right is particularly related to school institutions, symbol of guaranteed access and a means of promoting equality.

Given the importance of school institutions, in the late 1950s academics started the development of studies to understand the complexity of factors influencing student achievement and how schools can make a difference. This was the beginning of educational effectiveness research (EER). This field seeks to investigate the factors within schools and within educational systems that might affect the student outcomes.

The majority of the large-scale international studies has attempted to model the effects of a large number of factors by the input-process-output model in a search for "what works" in a various educational contexts (Creemers, Kyriakides, \& Sammons, 2010). In short, the results so far have shown the importance of students' family background, teachers' expectation, classroom climate, effective use of class time, adaptive learning, leadership, clear objectives, school resources, among others (Hattie, 2012; Karino, 2016; Sammons, Hillman, \& Mortimore, 1995).

There is already a great amount of evidence about many factors that influence the development and achievement of students. Although we need to assume that depending on the context the factors can vary. For example, research conducted in Latin America have highlighted the importance of other factors as school resources, school facilities and access to didactic material, factors not that important in places as the USA, Europe and Australia (Albanez, Ferreira, \& Franco, 2002; Murillo \& Roman, 2011). Therefore, depending on the level of the educational system, some factors will be more strongly associated with student learning. 
However, regardless of the level of the educational system and how strongly some factors are related to the output, there are some elements that every educational system should have. Considering this assumption, instead of trying to find factors that statistically demonstrate school effectiveness, the approach in this study is to build measures for the principal elements that all schools should have, allowing it to evaluate their quality. Even because it is necessary to consider that the association between contextual factors and student learning is affected by the variability of these factors in the population, as well as the measurement quality and methodological difficulties.

From this perspective, Willms (2015) developed the educational prosperity model. The term educational prosperity refers to the success of the education system in developing children's literacy skills and well-being, defined broadly in terms of their cognitive, social, emotional, physical, and spiritual well-being. The term is analogous to the term 'economic prosperity', usually associated with a country's economic growth.

The educational prosperity's framework follows a life-course approach until the age of 15 and beyond, based on the developmental stages, which lead to success: reading, mathematics, science skills, and non-cognitive outcomes. The prosperity outcomes at each stage of development are driver factors for the outcomes at subsequent stages. Difficulties to achieve the key outcomes of each stage will decrease the potential for educational success. The assumption is that cumulative intervention effects could recover up to $50 \%$ of the potential lost during the previous stages (Willms, Durepos, López García, Laurie, \& Tramonte, 2015).

The advantage of building measures is the possibility of monitoring. The measures allow us to synthesize and understand an educational system, and enable the setting of targets. The generation of information is the main objective of large-scale assessments (Inep, 2002; Karino, Vinha, \& Laros, 2014). Using the educational prosperity model as 
theoretical framework, it becomes possible to monitor the key elements of each stage and increase the likelihood of an adequate intervention.

Another contribution of this study is associated with the amount of evidence with respect to the validity and reliability of the measures. The large quantity of data collected in large scale assessments gains more value when transformed into reliable measurements. For Kyriakides (2008), more consistency and clear definitions of the measures are necessary. Reliability of the measurement scales seems to be less of an issue today than years ago, yet there is a concern related to the tendency of researchers to avoid assessing the validity of their measurement instruments (Hallinger \& Heck, 1996). This is alarming because all inferences made on basis of the results may be incorrect (Urbina, 2014).

Based on the educational prosperity approach, we have two goals with this study. First, we will propose and build measures for the educational prosperity approach and, secondly, describe and evaluate the Brazilian situation on each of the developed measures. We will use as reference the data of the Brazilian National Assessment of Educational Progress (Avaliação Nacional do Rendimento Escolar - ANRESC or Prova Brasil), intended for the second part of Brazilian elementary education.

\section{The Educational Prosperity model}

For the educational prosperity model, the family, the institution (school) and the community are the 'foundations for success', responsible to drive the outcomes. Specifically for the late primary and lower secondary stage (ages 9 to 15), the Educational Prosperity model defines four essential pillars related to institutions: (1) inclusive context, (2) quality instruction, (3) learning time and (4) human and material resources. These pillars are the core of the learning process and are focused on aspects that can be modified 
by the schools. The validity of the pillars rests on a large body of empirical results in EER that provides evidence of the relevance of these pillars (Willms et al., 2015).

Schools have an inclusive context when their environment enables all students to succeed. For UNESCO (2005), the inclusion is a process of addressing and responding to the diversity, which involves changes and modifications in content, approaches, structures and strategies, with a common vision to cover all children. 'All' means students from different religion, gender, sexual orientation, disabilities, ethnicities and social class.

The school must provide education for all and should be an example of respect to diversity. The development of metrics to measure inclusive environment should capture attitudes and practices of teachers and administrators, infrastructure to attend the diversity, politics of no segregation and the students' sense of belonging (Willms et al., 2015).

The quality of instruction is a proximal pillar, which means that the pillar has direct effects on student achievement and there are no mediating factors between the pillar and the outcome. Although everyone recognizes the importance of quality of instruction, it is very difficult to define and measure this construct. In the EER studies, the quality of instruction has been commonly measured using: teachers' education and characteristics, disciplinary climate, leadership, expectations, pedagogical resources (Sammons, Hillman, \& Mortimore, 1995; Wayne \& Youngs, 2003).

Most of these measures are distal factors; they not represent the core elements of quality instructions. For Anderson (2004), the 'delivery of the lessons' and 'interacting with students' are the center of the definition of teacher effectiveness. These factors are associated with the form and the quality of the lessons, concerning with the form of transmission of information, with the interaction and the feedback (Muijs, Kyriakides, Van der Werf, Creemers, Timperley, \& Earl, 2014). Moreover, Anderson (2004) adds that other 
factors mediate these two main factors: (1) standardized and structured learning units, (2) classroom climate and (3) classroom organization and management.

The learning time refers to the time on tasks, the amount of time that students are engaged in learning. In classrooms where there is indiscipline, the learning time is shorter, which means less opportunity to learn. Effective teachers are expected to organize and manage the classroom environment to maximize engagement (Kyriakides, 2008). Therefore, the learning time is also related to quality of instruction.

For Willms et al. (2015), learning time in low- and middle-income countries differs from that of high-income because it is more common the case of children who start school at a later age, they miss many days of school and they are more likely to repeat grades and work in part-time jobs. For this reason, we will try to capture aspects of learning time in and out of school.

School resources are the most objective pillar to be measured. This pillar includes aspects such as equipment, infrastructure, didactic facilities and human resources not complained in the quality of instruction and inclusive context pillars. A number of studies have suggested that this pillar does not impact on student achievement (Sammons, Hillman, \& Mortimore, 1995). However, this is an important pillar that should be measured to have a view as a whole of an educational system. Moreover, in low- and middle-income countries, material resources do have substantial effects (Murillo \& Roman, 2011).

The second and third 'foundations for success' are the family and community. Previous research has demonstrated relations between family and community and effective schools. Parental involvement is likely to lie in areas as: synchronizing school and home demands on students; assisting with homework; and giving feedback to the school on pupil progress and problems (Milner IV, 2013; Reynolds \& Teddlie, 2000). There are other 
studies that have failed to find this positive relationship, when parental involvement was based on criticism of the school (Teddlie \& Stringfield, 1993). It is the type of involvement that defines if there is a positive or negative relation with achievement. Here, the family involvement is related to helping in the classroom, giving support and creating opportunities.

The community support is very relevant, mainly in the poorest regions (Milner IV, 2013). As an extension of the family and the school, the community involvement contributes to the socialization and provides access to resources that support the educational progress (Small \& Newman, 2001). This could include literacy and recreation programs, facilities, and interventions to combat violence (Willms et al., 2015).

In addition to the three foundations described in the educational prosperity model, we will try to build measures for two other important and recurring literature factors in EER: socioeconomic level (SES) and student attainment. SES refers to the position of the student's family in a hierarchical social structure. It has been typically measured using income, level of education, and occupational prestige (NCES, 2012). Sometimes, the income is assessed from the family's property and possessions. Student attainment, in turn, relates to the life-course pathway of the student that has taken him to his current level of schooling (Willms et al., 2015).

These two factors cannot be directly modified by the school, but they tell a lot about the student context and his history. Furthermore, many studies validate the influence of these factors in student achievement (Fletcher, 1998; Laros, Marciano, \& Andrade, 2010; Teddlie, Stringfield, \& Reynolds, 2000). Because of all these reasons, a good evaluation of an educational system requires knowledge about these two factors.

To achieve the first goal of this study, we will build measures for all of the pillars of the educational prosperity model. As the Prova Brazil questionnaires were not elaborated 
using the same theoretical conception, we know that it is likely that we will not find good measures for all the pillars and, in some cases, distal measures will need to be used. With the measures, we hope to evaluate how much the Brazilian educational system is providing the essential for the development of their students.

\section{Method}

\section{Sample}

The study was conducted by using the database of a Brazilian national assessment, named Prova Brasil, administered in 2009. This large scale assessment was created in 2005 with the main purpose of evaluating all students of the elementary public schools in Brazil. With all the schools being evaluated, the government could trace a strategy of monitoring and goals for each school. The Prova Brasil measures the domains in Language and Mathematics, besides social and contextual factors.

The development of measures for the educational prosperity approach was based on the questionnaires developed for the grade 9 students of elementary school. The Prova Brasil questionnaires aim to access information about the socioeconomic and cultural level of the population, in addition to addressing family and school issues. The questionnaires are answered by students, teachers, principals and an external appraiser.

\section{Procedures}

First, all the variables of the Prova Brasil questionnaire were analyzed with the objective to identify those that could contribute to the pillars of the Educational Prosperity model. After the variables had been selected, we proceeded with the evaluation of their psychometric quality and descriptive analyses were executed. For the analyses of the variables, we considered only the individuals that answered at least $70 \%$ of the variables selected for the model. The intention was to exclude the individuals that did not answer the questionnaire properly. 
When variables could be combined to constitute a factor, we used Item Response Theory (IRT) to construct a scale. Two models were used during the analysis: twoparameter logistic (2PL) (Birnbaum, 1968) and graded response (Samejima, 1997), depending of characteristic of the item. Sometimes, these item response models were used mixed within a scale.

For the parameter calibration by IRT, we selected a sample of 3,000 students and 3,000 schools based on the ability level in math. This criterion was used because for the calibration it is important to have answers from students and schools of different proficiency levels. So, the math proficiency scale was divided in 10 equal scale points (decis) and 300 students were randomly selected in each of these scale points. The same procedure was executed for the schools, using the mean in math of the school. For the sample of teachers, we randomly selected one teacher among all those who answered the questionnaires of the selected schools.

The adjustment of the items to the unidimensional model was analyzed by the ratio between chi-square $\left(\chi^{2}\right)$ and the degrees of freedom. This is a general measure of adjustment and the criterion for acceptable fit is a ratio lower than 1.96 (Ullman, 2007). Another way to analyze the quality of the scale is by checking the local dependence of the items. The absence of local dependence is one of the assumptions of IRT. The local dependence was verified by the chi-square $\left(\chi^{2}\right)$ analysis, comparing the observed and expected frequencies in each of the two-way cross tabulations between responses to each item and each of the other items (Chen $\&$ Thissen, 1997). The value of $\chi^{2}$ becomes large if the pair of items indicates local dependence. According to IRT-PRO Guide, the criterion is a value lower than 2 or 3, but values lower than 10 are acceptable.

Once the scale was developed, interpretation of the scale was accomplished using psychometric foundations of the bookmark approach (Cizek, Bunch, \& Koons, 2004). The 
items of each scale were ordered by difficulty (easiest items first and hardest items last)

and, based on pedagogical and contextual judgment, we sought to identify the item that

represents a level change. Then, the item difficulty was translated into cut scores by noting

the examinee ability associated with .67 probability of a correct response on the item that

represents the change. The analyses were executed using Statistical Package for the Social

Sciences 19.0 (Nie, Bent, \& Hull, 1968) and IRT-PRO 2.1 (Cai, Thissen, \& Toit, 2011).

\section{Measures}

Table 1 shows the variables selected of each questionnaire for the development of the measures for the Educational Prosperity model.

Table 1

Description of the measures used to represent each pillar of the Educational Prosperity model.

\begin{tabular}{|c|c|c|c|c|}
\hline Pillars & Measures & Description & n items & Instrument \\
\hline \multirow{11}{*}{$\begin{array}{l}\text { Quality } \\
\text { Instruction }\end{array}$} & Teacher math homework & frequency of teacher correcting homework & 1 & Student \\
\hline & Adequacy of curriculum & the curriculum is appropriate & 1 & Teacher \\
\hline & Pedagogical practices & frequency of some pedagogical practices & 10 & Teacher \\
\hline & Teacher education & educational level of the teacher & 1 & Teacher \\
\hline & Principal education & educational level of the principal & 1 & Principal \\
\hline & Extra activities (sports) & school offers sports as extra activity & 1 & Principal \\
\hline & Extra activities (arts) & school offers arts as extra activity & 1 & Principal \\
\hline & Curriculum fulfillment & $\%$ of the content that was accomplished & 1 & Teacher \\
\hline & Didactical material & $\%$ of students that have didactical material & 1 & Teacher \\
\hline & Material on time & the didactical material arrives on time & 1 & Teacher \\
\hline & Quality material & the quality level of the didactical material & 1 & Teacher \\
\hline $\begin{array}{l}\text { Inclusive } \\
\text { Context }\end{array}$ & Selection test & school uses selection test to admit students & 1 & Principal \\
\hline \multirow{7}{*}{$\begin{array}{l}\text { Learning } \\
\text { time }\end{array}$} & Indiscipline of students & indiscipline is a problem in the classroom & 1 & Teacher \\
\hline & Teachers missing & teachers missing classes is a problem & 1 & Teacher \\
\hline & Students missing & students missing classes is a problem & 1 & Teacher \\
\hline & Tutoring activities & school offers tutoring activities & 1 & Principal \\
\hline & Do math homework & student do the math homework & 1 & Student \\
\hline & Domestic chores & time spent on chores in school days & 1 & Student \\
\hline & Work & student works in part-time jobs & 1 & Student \\
\hline \multirow{3}{*}{$\begin{array}{l}\text { School } \\
\text { Resources }\end{array}$} & Infrastructure conditions & condition of the school infrastructure & 14 & School \\
\hline & Equipment & presence of some equipment in the schools & 11 & School \\
\hline & Human resources & perception of human resources problems & 4 & Principal \\
\hline SES & SES & family possession and parents' education & 12 & Student \\
\hline \multirow{5}{*}{$\begin{array}{l}\text { Family and } \\
\text { Community } \\
\text { support }\end{array}$} & Mother - reading & student sees the mother reading & 1 & Student \\
\hline & Father - reading & student sees the father reading & 1 & Student \\
\hline & Books at home & number of books at home & 1 & Student \\
\hline & Community participation & community participates in school activities. & 8 & Principal \\
\hline & Family support & parents participate in school activities & 6 & Student \\
\hline \multirow{4}{*}{ Attainment } & Dropout & student dropped out of school & 1 & student \\
\hline & Failure & student was reproved & 1 & student \\
\hline & Starting school & when the student started to go to school & 1 & student \\
\hline & Correct age & student`s age corresponds with correct grade & 1 & student \\
\hline
\end{tabular}




\section{Results and Discussion}

\section{Quality of instruction}

Ten variables and one scale were used to represent this pillar. The scale, called pedagogical practices, was based on 10 items that assess the frequency of certain activities that were executed by math teachers in the classroom. The results of the first analysis demonstrated high local dependence among the items, a bad model fit and many answer categories showed unsatisfying differentiation.

Many trials were executed to improve the adjustment. The best fit was achieved after eliminating the following items: two items showing similar content with other items that had better discrimination, two items with the highest local dependence, and one item with low discrimination. Furthermore, we changed the answer categories of the items. Initially, the items had five answer categories varying between never and weekly. We transformed the five answer categories to two, choosing the category with the minimum desirable frequency that any practice should be executed in classroom as 1 (correct). The desirable frequency (presented between brackets in Table 2) was based on the item graphic analysis.

Table 2

Description and parameters of the items - scale of pedagogical practices.

\begin{tabular}{lccc}
\hline Description of the item & $\chi^{2} / \mathrm{df}$ & $a$ & $b_{1}$ \\
\hline do activities to automate procedures & - & - & - \\
deal with problems that require different reasoning & - & - & - \\
talk about possible solutions for a problem (sometimes per month) & 0.9 & 1.6 & -2.4 \\
memorize the rules needed for a solution & - & - & - \\
deal with everyday mathematical problems (weekly) & 1.3 & 1.4 & 1.0 \\
interpret numerical results (weekly) & 3.5 & 4.6 & -0.4 \\
deal with common problems & - & - & - \\
test different ways to resolve a problem (weekly) & 5.7 & 3.1 & -0.4 \\
improve the accuracy and speed of execution (once a month) & 5.9 & 3.1 & -1.0 \\
experiment different actions to resolve a problem & - & - & - \\
\hline
\end{tabular}


Table 2 shows the item parameters of the final scale of pedagogical practices. In this final scale, no more problems with local dependence were noticed, but some items still showed adjustment problems $\left(\chi^{2}\right)$.

After the interpretation, the pedagogical practice scale was composed by three levels. Table 3 presents the levels and the percentage of schools in each level. The average of the school on this scale is the mean proficiency estimated for each teacher of the school. The majority of the schools is located in level 2 , which means that the teachers in these schools probably work with the improvement of the accuracy and speed of math execution once a month and interpret numerical results and test different ways to resolve a problem at least every week.

The content analysis of the scale shows that it is not a good scale. Many important tasks of a good teacher are not covered. Another observation is that teachers that execute these activities in the desirable frequency are necessarily good teachers. In this way, we notice problems of content validity of this scale.

Table 3

Interpretation and percentage of school per level - scale of pedagogical practices.

\begin{tabular}{|c|c|c|c|}
\hline Level & Proficiency range & Interpretation & $\%$ Schools \\
\hline 1 & $<-0.8$ & $\begin{array}{l}\text { At this level, the teachers use the pedagogical practices in } \\
\text { a frequency lower than what is expected or talk about } \\
\text { possible solutions for a problem only sometimes during } \\
\text { the month. }\end{array}$ & 22.6 \\
\hline 2 & -0.8 to 1.0 & $\begin{array}{l}\text { At this level, the teachers probably work with the } \\
\text { improvement of the accuracy and speed of math execution } \\
\text { once a month and interpret numerical results and test } \\
\text { different ways to resolve a problem at least every week. }\end{array}$ & 58.9 \\
\hline 3 & $>1.0$ & $\begin{array}{l}\text { At this level, the teachers additionally deal with } \\
\text { mathematical everyday problems with their students every } \\
\text { week. }\end{array}$ & 18.5 \\
\hline
\end{tabular}

Beyond the pedagogical practice scale, other ten variables of the Prova Brazil questionnaires can represent this pillar. Table 4 shows the frequency of answers, missing data, skewness and kurtosis for each variable. Many interesting variables were found in 
Prova Brazil questionnaires like percentage of curriculum fulfillment, qualification of the principal and teachers, and quality of didactical material.

In short, there are some positive results that should be highlighted. About $85 \%$ of the teachers usually or always give homework to the students, $77 \%$ of the teachers judge the curriculum as adequate, more than $95 \%$ of the teachers and principals completed college and a substantial percentage of schools offer extra activities like art and sport.

Nonetheless, there are characteristics that should be improved. Only $28.7 \%$ of the teachers reported having completed more than $80 \%$ of the curriculum at the end of the year. A high percentage of students did not receive the didactical material on time (18.8\%), and more than $13 \%$ of the teachers reported that half or less than a half of the students had the didactical material to study. The quality of the didactical material is another important factor that needs to be mentioned. Only $15.2 \%$ of the teachers judged the didactical material as of very good quality.

These results show a great deal about the quality of instruction in the Brazilian educational system. The curriculum content is a way to measure the opportunity to learn (Muijs et al., 2014). As presented, in Brazil, problems with didactical material and delivery of the lessons are barriers for learning. In terms of measurement, the questionnaires do not inform about the 'interacting with students', 'learning units definition' and 'classroom organization', other important factors highlighted by Anderson (2004) and Hattie (2012) to understand teacher effectiveness. 
Table 4

Frequency of answers, missing data, skewness and kurtosis - quality of instruction variables.

\begin{tabular}{|c|c|c|c|c|}
\hline Measures & $\%$ Category & $\%$ Missing & Skewness & Kurtosis \\
\hline Teacher gives homework math & & 1.1 & -2.435 & 5.366 \\
\hline 0 (never or almost never) & 10.9 & & & \\
\hline 1 (usually) & 40.2 & & & \\
\hline 2 (always or almost always) & 47.9 & & & \\
\hline Adequacy of curriculum & & 2.3 & -1.407 & -.020 \\
\hline $0-$ no & 20.7 & & & \\
\hline $1-$ yes & 77.0 & & & \\
\hline Education of teacher & & 6.1 & -.040 & .230 \\
\hline less than high school & 0.1 & & & \\
\hline high school & 3.0 & & & \\
\hline College & 43.1 & & & \\
\hline college + specialization (360 hours) & 45.6 & & & \\
\hline college + master degree & 1.8 & & & \\
\hline college + doctor degree & 0.2 & & & \\
\hline Education of principal & & 5.0 & -.660 & .764 \\
\hline less than high school & 0.1 & & & \\
\hline high school & 4.4 & & & \\
\hline College & 30.1 & & & \\
\hline college + specialization (360 hours) & 58.5 & & & \\
\hline college + master degree & 1.6 & & & \\
\hline college + doctor degree & 0.3 & & & \\
\hline Extra school activities (sports) & & 1.0 & -1.268 & -.391 \\
\hline $0-$ no & 23.0 & & & \\
\hline $1-$ yes & 76.0 & & & \\
\hline Extra school activities (arts) & & 1.5 & -.510 & -1.740 \\
\hline $0-$ no & 37.3 & & & \\
\hline $1-$ yes & 61.7 & & & \\
\hline$\%$ Curriculum fulfillment & & 1.5 & -.562 & -.115 \\
\hline less than $40 \%$ & 4.5 & & & \\
\hline between $40 \%$ and $60 \%$ & 17.5 & & & \\
\hline between $60 \%$ and $80 \%$ & 47.8 & & & \\
\hline more than $80 \%$ & 28.7 & & & \\
\hline Didactical material & & 20.6 & -1.673 & 1.529 \\
\hline No & 6.9 & & & \\
\hline yes, less than a half of the students & 3.2 & & & \\
\hline yes, half of the students & 3.3 & & & \\
\hline yes, the most part & 15.8 & & & \\
\hline yes, everybody & 50.1 & & & \\
\hline Material arrives on time & & 19.7 & -1.256 & -.422 \\
\hline $0($ no) & 18.8 & & & \\
\hline 1 (yes) & 61.5 & & & \\
\hline Quality of didactical material & & 15.4 & -.930 & -.757 \\
\hline 0 (does not apply) & 4.8 & & & \\
\hline 1 (bad) & 4.2 & & & \\
\hline 2 (acceptable) & 19.9 & & & \\
\hline 3 (good) & 40.5 & & & \\
\hline 4 (very good) & 15.2 & & & \\
\hline
\end{tabular}




\section{Inclusive context}

It was quite difficult to find a metric for the pillar of inclusive context because the questionnaires of Prova Brazil 2009 did not include this construct as a focus. The only variable that approaches this pillar is the use of selection test to accept students. In the Brazilian public educational system, only $1.2 \%$ of the schools use selection test.

The Brazilian school system cannot be judged in terms of inclusive context based only on one variable. Because the evaluation of this pillar is much broader than segregation by ability, we suggest the future inclusion of new variables in the Prova Brasil questionnaires. In the present questionnaires, the following important aspects mentioned by Willms et al. (2015) were not included: attitudes and practices of teachers and administrators, infrastructure to attend the diversity and the students' sense of belonging.

\section{Learning time}

Learning time refers to the amount of time that students are engaged in learning. Examples of variables that measure learning time are number of school days, time doing homework and other activities. Another way to measure learning time is verifying if the school has problems with (1) indiscipline of students and (2) student and teacher missing classes. We suppose that when the school has these problems, students have consequently less time to learn.

The number of school days is not a good measure of learning time in Brazil because all the public school must comply a minimum of school days required by law. So, the difference of school days between schools is small. We found two variables in Prova Brasil questionnaires related to learning time: students doing homework and schools offering tutoring activities. Only $5 \%$ of the students assume that they never or almost never 
do homework and more than $75 \%$ of the schools develop activities to help the students with learning difficulties. These results are in favor of increasing learning time.

However, we found that almost $70 \%$ of the teachers consider student indiscipline as a problem. We have two conclusions based on this result. First, in Brazil, because of the indiscipline, learning time is not being fully availed, which means less opportunity to learn. Second, this result also shows problems with the quality of instruction. For Kyriakides (2008), an effective teacher should be able to organize and manage the classroom environment to maximize engagement. Indiscipline provides evidence that the engagement has not been achieved.

Finally, others important results are: (1) in more than $30 \%$ of the schools, teachers missing classes is a problem; (2) in more than $40 \%$ of the schools, students missing classes is a problem, (3) more than $15 \%$ of the students spend more than 2 hours in domestic chores per week and (4) $18.9 \%$ of the students work in part-time jobs. These results are evidence that there are many factors that do not contribute to learning time in Brazilian educational system. 
Table 5

Frequency of answers, missing data, skewness and kurtosis - learning time variables.

\begin{tabular}{|c|c|c|c|c|}
\hline Measures & $\%$ Category & $\%$ Missing & Skewness & Kurtosis \\
\hline Indiscipline of the students in the classroom & & 2.1 & -.936 & -1.124 \\
\hline 0 (no, it isn't a problem) & 28.2 & & & \\
\hline 1 (yes, it is a problem) & 69.7 & & & \\
\hline Teachers missing classes & & 17.0 & 1.069 & -.242 \\
\hline 0 (no, it isn't a problem) & 52.1 & & & \\
\hline 1 (yes, it is a problem) & 20.4 & & & \\
\hline 2 (yes, it is a serious problem) & 10.5 & & & \\
\hline Students missing classes & & 16.0 & .684 & -.795 \\
\hline 0 (no, it isn't a problem) & 42.9 & & & \\
\hline 1 (yes, it is a problem) & 29.3 & & & \\
\hline 2 (yes, it is a serious problem) & 11.8 & & & \\
\hline Student do math homework & & 1.9 & -.825 & -.316 \\
\hline 0 (never or almost never) & 5.0 & & & \\
\hline 1 (usually) & 36.6 & & & \\
\hline 2 (always or almost always) & 55.6 & & & \\
\hline Tutoring activities & & 0.5 & $-1,271$ & -.385 \\
\hline 0 (no) & 23.1 & & & \\
\hline 1 (yes) & 76.4 & & & \\
\hline Domestic chores & & 1.3 & .628 & -.299 \\
\hline 0 (no) & 18.2 & & & \\
\hline 1 (1 hour or less) & 38.9 & & & \\
\hline 2 (between 1 and 2 hours) & 23.5 & & & \\
\hline 3 (between 2 and 3 hours) & 10.7 & & & \\
\hline 4 (3 hours or more) & 7.5 & & & \\
\hline Work in part-time jobs & & 5.6 & 1.497 & .242 \\
\hline 0 (no) & 75.5 & & & \\
\hline 1 (yes) & 18.9 & & & \\
\hline
\end{tabular}

\section{School resources}

In a good educational system, the absence of school resources should not be a barrier to teaching and learning. Therefore, minimum conditions should be ensured to provide a learning environment. Based on Prova Brasil questionnaires, three scales were built to represent this pillar: infrastructure conditions, equipment and human resources.

The scale of infrastructure conditions assessed the quality (bad, regular or good) of some essential school items, like: classrooms, walls, and washrooms. Only ventilation and illumination of the classrooms were assessed as present or absent. Table 6 presents all items of this scale. Four items with local dependence problems were excluded of the analysis. 
Table 6

Description and parameters of the items - scale of infrastructure conditions.

\begin{tabular}{lccccc}
\hline Item description & $\chi^{2} / \mathrm{df}$ & $a$ & $b_{1}$ & $b_{2}$ & $b_{3}$ \\
\hline classroom & 1.48 & 3.38 & -4.18 & -1.53 & -0.18 \\
wall & 1.28 & 2.60 & -4.51 & -1.72 & -0.38 \\
floor & 1.80 & 2.11 & -3.84 & -1.39 & -0.21 \\
electric structure & 1.72 & 1.92 & -4.13 & -1.07 & 0.18 \\
roof & 1.49 & 1.75 & -4.49 & -1.54 & -0.28 \\
hydraulics & - & - & - & - & - \\
school entrance & 1.14 & 1.90 & -4.00 & -1.73 & -0.38 \\
doors & 1.78 & 2.21 & -3.31 & -1.20 & 0.07 \\
hall & - & - & - & - & - \\
washroom & 1.90 & 2.13 & -3.82 & -0.97 & 0.32 \\
patio & - & - & - & - & - \\
kitchen & 1.58 & 1.66 & -3.60 & -1.63 & -0.28 \\
windows & - & - & - & - & - \\
ventilated classroom & 1.18 & 1.18 & -1.37 & - & - \\
classroom illumination & 1.48 & 1.32 & -2.16 & - & - \\
\hline
\end{tabular}

For the purpose of interpretation, the scale was divided into four levels. In the first level, we grouped the schools that probably indicated bad conditions for the most part of the items assessed. In the second level, the schools indicated some items with regular conditions and, in the third level, there is a larger number of items in regular conditions. Most of the schools are in the fourth level (50.3\%). These schools have a high probability of having good conditions in all investigated items.

Table 7

Interpretation and percentage of school per level - scale of infrastructure conditions.

\begin{tabular}{|c|c|c|c|}
\hline Level & Proficiency range & Interpretation & $\%$ Schools \\
\hline 1 & $<-1.5$ & $\begin{array}{l}\text { At this level, the schools don't have some elements of } \\
\text { infrastructure or have high probability of having bad } \\
\text { conditions of these elements: walls, roof, classrooms, } \\
\text { school entrance, kitchen, floor, electric structure, } \\
\text { washrooms and doors. }\end{array}$ & 5.7 \\
\hline 2 & -1.5 to -1.0 & $\begin{array}{l}\text { At this level, the schools have a high probability of } \\
\text { having a classroom with enough illumination and } \\
\text { regular conditions of the classroom, walls, school } \\
\text { entrance and kitchen. }\end{array}$ & 9.3 \\
\hline 3 & -1.0 to 0.0 & $\begin{array}{l}\text { At this level, the schools have a high probability of } \\
\text { having a classroom with enough illumination and } \\
\text { ventilation, and regular conditions of the classroom, } \\
\text { walls, school entrance, kitchen, roof, floor doors, } \\
\text { electric structure and washrooms. }\end{array}$ & 34.7 \\
\hline 4 & $>0.0$ & $\begin{array}{l}\text { At this level, the schools have high probability of } \\
\text { having good conditions of all investigated elements: } \\
\text { walls, school entrance, classroom, kitchen, floor, roof, } \\
\text { doors, electric structure and washrooms. }\end{array}$ & 50.3 \\
\hline
\end{tabular}


Another evaluated aspect was the possession of certain equipment by the school. Essential equipment for a good functioning of the school (like copy machine and fire safety equipment) and for the execution of pedagogical activities (like computer and overhead projector). Initially, the questionnaire had items evaluating the quality of the equipment (absent, bad, regular, good). However, during the analysis, this scale did not show a good fit. So, the items were transformed in dichotomous format (absent and present). Additionally, it was necessary to exclude three items because of the following reasons: local dependence (fax and slide projector) and extremely high discrimination parameter (computer).

Table 8

Description and parameters of the items - scale of equipment.

\begin{tabular}{lccc}
\hline Item description & $\chi^{2} / \mathrm{df}$ & $a$ & $b_{1}$ \\
\hline telephone & 2.73 & 2.60 & -0.86 \\
fax & - & - & - \\
printer & 0.98 & 2.31 & -2.22 \\
overhead projector & 2.34 & 1.57 & -1.26 \\
computer & - & - & - \\
sound equipment & 1.05 & 1.46 & -2.53 \\
copy machine & 1.61 & 1.11 & -0.58 \\
slide projector & - & - & - \\
fire safety equipment & 1.26 & 1.55 & 0.16 \\
VCR equipment & 1.57 & 0.78 & -1.87 \\
TV & 1.04 & 1.92 & -3.29 \\
\hline
\end{tabular}

Using the interpreted scale, we note that in level 1 schools only have basic equipment as TV, sound equipment and printer. There are $21.2 \%$ of the schools in Brazil in this level. These schools probably do not have VCR equipment, overhead projector, copy machine and telephone. Only $19 \%$ of the schools have high probability to have all the equipment investigated. 
Table 9

Interpretation and percentage of school per level - scale of equipment.

\begin{tabular}{cclc}
\hline Level & Proficiency range & \multicolumn{1}{c}{ Interpretation } & \% Schools \\
\hline 1 & $<-1.0$ & $\begin{array}{l}\text { At this level, the schools probably have TV, sound } \\
\text { equipment and printer. }\end{array}$ & 19.4 \\
\hline 2 & -1.0 to 0.0 & $\begin{array}{l}\text { At this level, the schools additionally have VCR } \\
\text { equipment and overhead projector. }\end{array}$ & 31.3 \\
\hline 3 & 0.0 to 0.7 & $\begin{array}{l}\text { At this level, the schools additionally have copy } \\
\text { machine and telephone. }\end{array}$ & 26.6 \\
\hline 4 & & $\begin{array}{l}\text { At this level, the schools additionally have fire } \\
\text { safety equipment. }\end{array}$ \\
\hline
\end{tabular}

These results demonstrate that schools in Brazil have different levels of infrastructure conditions and possession of equipment. We also highlight that both scales are simple, not very demanding scales that assess basic resources. Therefore, we perceived an educational system that works with scarce material resources and problems in terms of equity.

School resources also include human resources. One section of the Prova Brasil questionnaires evaluates the perceived problem of human resources by the school principal. Only one item out of the five present in the questionnaire showed problems with adjustment and local dependence.

Table 10

Description and parameters of the items - scale of human resources problems.

\begin{tabular}{lccc}
\hline Item description & $\chi^{2} / \mathrm{df}$ & $a$ & $b_{1}$ \\
\hline lack of teachers & 0.9 & 2.2 & -0.3 \\
lack of administrative staff & 1.5 & 1.2 & -0.3 \\
turnover of teachers & 1.0 & 1.2 & 0.4 \\
high absence rate (teachers) & 1.0 & 1.2 & 0.3 \\
lack of pedagogical support & - & - & - \\
\hline
\end{tabular}

The results in Table 11 show that $59.3 \%$ of the principals do not discern problems related to human resources in their schools. Such a high rate was not expected due to a number of studies reporting problems related to quantity and quality of teachers in Brazil 
(Gatti, 2010; Sampaio \& Marin, 2004). One interpretation for this rate is social desirability. The principals may have responded in order to transmit the impression of a better school.

Among the perceived problem of human resources, the most common are lack of teachers and lack of administrative staff. These problems were reported by a little over $30 \%$ of the principals. In level 3, composed by $9.7 \%$ of the schools, the principals perceived other problems as high absence rate and turnover of teachers.

Table 11

Interpretation and percentage of schools per level - scale of human resources problems.

\begin{tabular}{|c|c|c|c|}
\hline Level & Proficiency range & Interpretation & $\%$ Schools \\
\hline 1 & $<0.0$ & $\begin{array}{l}\text { In this level, the principals do not perceive the } \\
\text { investigated human resources aspects as a } \\
\text { problem. }\end{array}$ & 59.3 \\
\hline 2 & 0.0 to 1.0 & $\begin{array}{l}\text { In this level, the principals probably perceive that } \\
\text { lack of teachers and lack of administrative staff are } \\
\text { human resources problems. }\end{array}$ & 30.9 \\
\hline 3 & $>1.0$ & $\begin{array}{l}\text { In this level, the principals probably perceive that } \\
\text { lack of teachers, lack of administrative staff, high } \\
\text { absence rate and turnover of teachers are human } \\
\text { resources problems in the school. }\end{array}$ & 9.7 \\
\hline
\end{tabular}

\section{Socioeconomic level and poverty}

The measure of socioeconomic level was constructed using nine items related to the family property (number of TVs, cars, others), one item about the presence of a maid in the family and two items associated to the education of the parents. This indicator is based on the same methodological approach proposed by INEP (INEP, 2014). The item parameters are presented in Table 12. Only one item presented fit problems. No local dependence was observed. 
Table 12

Description and parameters of the items - scale of socioeconomic level.

\begin{tabular}{lcccccc}
\hline Item description & $\chi^{2} / \mathrm{df}$ & $a$ & $b_{1}$ & $b_{2}$ & $b_{3}$ & $B_{4}$ \\
\hline computer/internet & 0.84 & 2.1 & 0.2 & - & - & - \\
$\mathrm{TV}$ & 1.81 & 1.8 & -2.7 & -0.1 & 1.3 & - \\
car & 0.93 & 1.5 & 0.4 & 2.1 & 3.3 & - \\
bathroom & 0.95 & 1.7 & -2.2 & 1.0 & 2.4 & 3.33 \\
washing machine & 0.94 & 1.6 & -0.2 & - & - & - \\
freezer & 0.56 & 0.8 & 2.0 & - & - & - \\
radio & 1.02 & 1.0 & -2.6 & 0.8 & 2.9 & - \\
refrigerator & 2.22 & 1.5 & -2.6 & 2.0 & - & - \\
DVD player & 1.20 & 1.7 & -1.7 & - & - & - \\
maid & 1.85 & 0.9 & 2.9 & 3.8 & - & - \\
mother's education & 1.30 & 0.8 & -1.5 & 1.4 & 3.9 & - \\
father's education & 1.38 & 0.8 & -0.6 & 1.9 & 4.2 & - \\
\hline
\end{tabular}

In Table 13, the scale interpretation and the percentage of students in each level are presented. In the first level, which represents almost $30 \%$ of the students, the families probably have in their houses: one TV, one refrigerator, one bathroom, one radio and one DVD player. The parents of the students in level 1 probably did not complete the elementary school. Only $0.3 \%$ of the students have parents who probably completed at least high school.

Table 13

Interpretation and percentage of students per level-scale of socioeconomic level.

\begin{tabular}{|c|c|c|c|}
\hline Level & Proficiency range & Interpretation & $\%$ Students \\
\hline 1 & $<-0.5$ & $\begin{array}{l}\text { In this level, the students probably have one TV, one } \\
\text { refrigerator, one bathroom, one radio and one DVD } \\
\text { player in their house. }\end{array}$ & 28.8 \\
\hline 2 & -0.5 to 0.5 & $\begin{array}{l}\text { In this level, the students probably have one TV, one } \\
\text { refrigerator, one bathroom, one radio, one DVD player } \\
\text { and additionally one washing machine in their house } \\
\text { and the mother completed elementary school. }\end{array}$ & 41.5 \\
\hline 3 & 0.5 to 1.0 & $\begin{array}{l}\text { In this level, the students probably start to have two TVs } \\
\text { and one computer in their house, and the father also } \\
\text { completed elementary school. }\end{array}$ & 16.3 \\
\hline 4 & 1.0 to 2.5 & $\begin{array}{l}\text { In this level, the students probably start to have two } \\
\text { radios, three or more TVs, one car and two bathrooms in } \\
\text { their house. }\end{array}$ & 13.0 \\
\hline 5 & $>2.5$ & $\begin{array}{l}\text { In this level, the students probably start to have three or } \\
\text { more radios, two or more cars, three or more bathrooms, } \\
\text { two or more refrigerators, a freezer and a maid in their } \\
\text { house, and the parents completed at least high school. }\end{array}$ & 0.3 \\
\hline
\end{tabular}




\section{Family and community support}

Family support was assessed using six items related to parental involvement during school activities. Only one item had adjustment problems. However, we decided to keep the item in the scale (Table 14).

Table 14

Description and parameters of the items - scale of family support.

\begin{tabular}{lcccc}
\hline Item description & $\chi^{2} / \mathrm{df}$ & $a$ & $b_{1}$ & $b_{2}$ \\
\hline parents go to school meetings & 1.45 & 0.9 & -3.0 & -0.6 \\
parents encourage studying & 0.46 & 2.9 & -2.5 & - \\
parents encourage doing homework & 1.56 & 2.9 & -1.9 & - \\
parents encourage Reading & 0.71 & 3.0 & -1.6 & - \\
parents encourage going to school/not missing classes & 0.44 & 2.3 & -2.7 & - \\
parents talk about what is happening in school & 3.25 & 1.5 & -1.0 & - \\
\hline
\end{tabular}

Based on the scale interpretation, we noticed that the most part of the students (levels 2 and 3) has parents who recognize the importance of school, encourage studying, and frequently attend school meetings. These results are positive, but it is necessary to considerer that the scale is simple, not very demanding.

Table 15

Interpretation and percentage of students per level - scale of family support.

\begin{tabular}{cclc}
\hline Level & Proficiency range & \multicolumn{1}{c}{ Interpretation } & $\%$ Students \\
\hline 1 & $<-2$ & $\begin{array}{l}\text { In this level, the students have parents that } \\
\text { probably encourage studying, going to school and } \\
\text { not missing classes. }\end{array}$ & 1.4 \\
\hline & & $\begin{array}{l}\text { In this level, the students have parents that } \\
\text { additionally encourage reading and doing the } \\
\text { homework, talk about what is happening on the } \\
\text { school and usually attend the school meeting. }\end{array}$ & 31.9 \\
3 & & $\begin{array}{l}\text { In this level, the students have parents that } \\
\text { encourage their children, talk about what is } \\
\text { happening on the school and always attend the } \\
\text { school meeting. }\end{array}$ \\
\hline
\end{tabular}

Originally, there were eight items to evaluate community support. However, we had problems with the local dependence of some items because they measured the same content with small differences. In these cases, we decided to join the common items and treat them as item parcels. The joined items were: two items related to task force (cleaning 
or maintaining the physical structure); two items related to solidarity campaign (organized by community or by school); and two items related to events in school (organized by community or by others). When only one of the situations occurred, one point was attributed. When both situations occurred, two points were attributed. So, in the end, five items were used to build the community support scale. One of these items presented local dependence and it was excluded of the scale.

Table 16

Description and parameters of the items - scale of community support.

\begin{tabular}{lcccc}
\hline Item description & $\chi^{2} / \mathrm{df}$ & $a$ & $b_{1}$ & $b_{2}$ \\
\hline task force: cleaning or maintaining the physical structure & 1.9 & 1.7 & 1.0 & 1.8 \\
maintenance of orchard and garden. & 1.1 & 1.4 & 1.6 & - \\
solidarity campaign: organized by community or by school & 1.9 & 0.9 & -1.2 & 0.7 \\
events in school: organized by community or by others & 1.3 & 0.6 & -2.5 & 0.4 \\
school events for community & - & - & - & - \\
\hline
\end{tabular}

Analyzing the interpreted scale, we noted that the presence of the community in the schools as promoting events and solidarity campaign (62.6\%) is common in Brazil. In $35.3 \%$ of the schools, the community also organizes task force related to cleaning the school or maintaining the physical structure. It is not clear whether the presence of the community has been complementary or palliative due to lack of a minimum structure for appropriate school functioning. In other words, these results can be positive or evidence of an educational system that needs community support to operate.

Table 17

Interpretation and percentage of schools per level - scale of community support.

\begin{tabular}{cccc}
\hline Level & Proficiency range & \multicolumn{1}{c}{ Interpretation } & $\%$ Schools \\
\hline 1 & $<0.0$ & $\begin{array}{l}\text { In the schools of this level, the community or others } \\
\text { promoted events and solidarity campaign. }\end{array}$ & 62.6 \\
\hline & $\begin{array}{l}\text { In the schools of this level, the community and others } \\
\text { promoted events and solidarity campaign, the } \\
\text { community also organized task force cleaning the } \\
\text { school or maintaining the structure. }\end{array}$ & 35.3 \\
3 & $\begin{array}{l}\text { In the schools of this level, the community and others } \\
\text { promoted events and solidarity campaign, the } \\
\text { community organized task force cleaning the school and } \\
\text { maintaining the structure and helping to maintain the } \\
\text { garden and orchard. }\end{array}$ & 2.0 \\
\hline
\end{tabular}




\section{Attainment}

Four questions in the Prova Brasil questionnaires assessed student attainment (Table 18). The frequency analysis shows that, in the Brazilian education system, $93.3 \%$ of the students never dropout and $78.2 \%$ of the students began their school career at pre-school or kindergarten. However, problems with grade repetition are evident and, consequently, there are also problems with the correspondence between student age and grade.

Table 18

Frequency of answers, missing data, skewness and kurtosis - attainment variables.

\begin{tabular}{|c|c|c|c|c|}
\hline Measures & $\%$ Category & $\%$ Missing & Skewness & Kurtosis \\
\hline dropout & & .8 & 4.531 & 21.443 \\
\hline $0($ no) & 93.3 & & & \\
\hline 1 (yes, one time) & 4.8 & & & \\
\hline 2 (yes, twice or more) & 1.2 & & & \\
\hline grade repetition & & 1.8 & 1.204 & .173 \\
\hline $0($ no $)$ & 64.3 & & & \\
\hline 1 (yes, one time) & 24.1 & & & \\
\hline 2 (yes, twice or more) & 9.8 & & & \\
\hline first school inscription & & 2.5 & -.744 & -.274 \\
\hline 1 (after grade 1 of Elementary school) & 3.1 & & & \\
\hline 2 (at grade 1 of Elementary school) & 16.1 & & & \\
\hline 3 (at pre-school) & 35.5 & & & \\
\hline 4 (at kindergarten) & 42.7 & & & \\
\hline correct age & & .0 & -1.202 & -.556 \\
\hline $0($ no) & 24.3 & & & \\
\hline 1 (yes) & 75.7 & & & \\
\hline
\end{tabular}

\section{Conclusions}

Educational effectiveness research (EER) was criticized to consist of a set of empirical studies, without a solid theory and criteria to insert variables in the model (Van den Eeden, Hox, \& Hauer, 1990). The educational prosperity model defines the essence of educational systems and enables the development of more theoretically driven research. The pillars of good education were defined considering the findings of the EER, but they were not restricted to these empirical results. The followed approach is very interesting, because the restriction to 'what works' empirically can result in a poor and limited theory. 
This study had two objectives: (1) develop measures for the educational prosperity approach and (2) evaluate the Brazilian situation on each of the developed measures. Considering the first goal, we conclude that, by using the Prova Brasil questionnaires, it was possible to build or find measures for the three foundations for success: family, school and community. In addition, measures were constructed for two other important factors: socioeconomic level (SES) and student attainment. Nevertheless, some pillars were not completely addressed, as in the case of inclusive context, or could have a more complete coverage.

It is necessary to consider the background in which the measures were constructed, the main limitation of this study. Given the theory of educational prosperity model, we have tried to develop good measures based on questions of Prova Brasil questionnaires. The construction of instruments specifically for this mean would have been ideal. Although the Prova Brasil questionnaires were not developed originally to attend this theory, they aimed to measure several constructs related to students, classes and schools, also based on the results in EER (Franco, Fernandes, Soares, Beltrão, Barbosa, \& Alves, 2003; Inep, 2002). Therefore, even though the instruments were not specifically developed to attend the theory of Educational Prosperity model, there are some common principles between the theoretical framework of Prova Brasil and the educational prosperity model.

Despite the limitation, good scales and items were found as representatives of the educational prosperity model, as well as important directions to improve the measures. Considering the characteristics of Brazil, the results of this study are particularly important for countries in economic development, like Latin American countries.

Considering the second objective of this study, we observed that the Brazilian educational system has equity problems and has aspects to improve in all assessed pillars. Even basic elements, such as infrastructure, are not provided to all students. These results 
can explain the low position of Brazil in international assessments, as in the PISA studies (Programme of International Student Assessment) (Carnoy, Khavenson, Fonseca, Costa, \& Marotta, 2015). The results obtained in this study on the newly developed measures make the educational situation in Brazil even more evident and also allow better comparability with other educational systems. To conclude, we hope that these results contribute to studies in the field of school effectiveness and to promote action in favor of the improvement of the Brazilian educational system.

\section{References}

Albanez, A., Ferreira, F., \& Franco, F. (2002). Qualidade e equidade no ensino fundamental brasileiro. Pesquisa e Planejamento Econômico, 32(3), 453-475.

Anderson, L. W. (2004). Increasing teacher effectiveness ( $2^{\text {nd }}$ edition). Paris: UNESCO International Institute for Educational Planning.

Birnbaum, A. (1968). Some latent trait models and their use in inferring an examinee's ability. In F.M. Lord \& M.R. Novick, Statistical theories of mental test scores (pp. 392-479). Reading, MA: Addison-Wesley.

Cai, L., Thissen, D., \& du Toit, S. (2011). IRTPRO 2.1 [Computer Program]. Skokie, IL: Scientific Software International, Inc.

Carnoy, M., Khavenson, T., Fonseca, I., Costa, L., \& Marotta, L. (2015). A educação brasileira está melhorando? Evidências do Pisa e do SAEB. Cadernos de Pesquisa, 4(257), 450-485.

Chen, W. H., \& Thissen, D. (1997). Local dependence indices for item pairs using item response theory. Journal of Educational and Behavioral Statistics, 22, 265-289.

Cizek, G. J., Bunch, M. B., \& Koons, H. (2004). Setting performance standards: Contemporary methods. Educational Measurement: Issues and Practices, 23(4), 3150 .

Creemers, B. P. M., Kyriakides, L., \& Sammons, P. (2010). Methodological advances in educational effectiveness research. London, New York: Routledge.

Fletcher, P. R. (1998). À procura do ensino eficaz. Relatório técnico. Brasília: MECDAEB.

Franco, C., Fernandes, C., Soares, J. F., Beltrão, K., Barbosa, M. E., \& Alves, M. T. G. (2003). O referencial teórico na construção dos questionários contextuais do SAEB 2001. Estudos em Avaliação Educacional, 28, 39-74. 
Gatti, B. A. (2010). Formação de professores no Brasil: Características e problemas. Educação e Sociedade, Campinas, 31(113), 1355-1379.

Hallinger, P., \& Heck, R. H. (1996). Reassessing the principal's role in school effectiveness: A review of the empirical research, 1980-1995. Educational Administration Quarterly, 32(1), 5-44.

Hattie, J. (2012). Visible learning for teachers: Maximizing impact on learning. London and New York: Routledge.

Instituto Nacional de Estudos e Pesquisas Educacionais (2002). SAEB 2001: Novas perspectivas. Brasília: Inep.

Instituto Nacional de Estudos e Pesquisas Educacionais (2014). Nota técnica: Indicador de Nível Socioeconômico das Escolas de Educação Básica (Inse). Brasília: INEP.

Karino, C. A. (2016). Estudos brasileiros sobre eficácia escolar: uma revisão de literatura. Manuscrito em preparação.

Karino, C. A., Vinha, L. G. do A., \& Laros, J. A. (2014). Os questionários do SAEB: O que eles realmente medem? Estudos em Avaliação Educacional, 25(59), 270-297.

Kyriakides, L. (2008). Testing the validity of the comprehensive model of educational effectiveness: A step towards the development of a dynamics model of effectiveness. School Effectiveness and School Improvement, 19(4), 429-446.

Laros, J. A., Marciano, J. L. P., \& Andrade, J. M. (2010). Fatores que afetam o desempenho na prova de matemática do SAEB: Um estudo multinível. Avaliação Psicológica, 9(2), 173-186.

Milner IV, H. R. (2013). Analysing poverty, learning, and teaching through a critical race theory lens. Review of research in education, 37(1), 1-53.

Muijs, D., Kyriakides, L., Van der Werf, G., Creemers, B., Timperley, H., \& Earl, L. (2014). State of the art - teacher effectiveness and professional learning. School Effectiveness and School improvement: An International Journal of Research, Policy and Practice, 25(2), 231-256.

Murillo, F. J., \& Roman, M. (2011). School infrastructure and resources do matter: Analysis of the incidence of school resources on the performance of Latin American students. School Effectiveness and School Improvement, 22(1), 29-50.

National Center for Education Statistics (2012). Improving the measurement of socioeconomic status for the national assessment of educational progress: A theoretical foundation. Washington, DC: NCES.

Nie, N. Hh., Bent, D. H., \& Hull, C. H. (1968). SPSS [Computer Program]. Armonk, New York: IBM Corporation. 
Reynolds, D., \& Teddlie, C. (2000). The process of school effectiveness. In C. Teddlie, \& D. Reynolds (Eds.), The international handbook of school effectiveness research (pp. 134-159). New York: Routledge.

Samejima, F. (1997). Graded response model. In W. van der Linden \& R.K. Hambleton (Eds.), Handbook of modern item response theory (pp. 85-100). N.Y.: Springer.

Sammons, P., Hillman, J., \& Mortimore, P. (1995). Key characteristics of effective schools: A review of school effectiveness research. London: Office for Standards in Education [OFSTED].

Sampaio, M. das M. F., \& Marin, A. J. (2004). Precarização do trabalho docente e seus efeitos sobre as práticas curriculares. Educação e Sociedade, 25(89), 1203-1225.

Small, M. L., \& Newman, K. (2001). Urban poverty after the truly disadvantaged: The rediscovery of the family, the neighborhood, and culture. Annual Review of Sociology, 27(1), 23-45.

Teddlie, C., \& Stringfield, S. (1993). Schools do make a difference: Lessons learned from a 10-year study of school effects. New York: Teachers College Press.

Teddlie, C., Stringfield, S., \& Reynolds, D. (2000). Context issues within school effectiveness research. In C. Teddlie, \& D. Reynolds (Eds.), The international handbook of school effectiveness research (pp. 160-185). New York: Routledge.

Ullman, J. B. (2007). Structural equation modeling. In B. G. Tabachnick \& L. S. Fidell (Eds.), Using multivariate statistics ( $5^{\mathrm{a}}$ ed., pp. 676-780). Boston: Pearson Education.

UNESCO (2005). Guidelines for inclusion: Ensuring access to education for all. Paris: UNESCO.

United Nations (1948). The universal declaration of human rights. Paris: Author.

Urbina, S. (2014). Essentials of psychological testing ( $2^{\text {nd }}$ edition). Hoboken, NJ: Wiley.

Van den Eeden, P., Hox, J., \& Hauer, J. (1990). Theory and model in multilevel research: Convergence or divergence? Amsterdam: SISWO.

Wayne, A. J., \& Youngs, P. (2003). Teacher characteristics and student achievement gains: A review. Review of Educational Research, 73(1), 89-122.

Willms, J. D. (2015). Educational prosperity. Fredericton, NB: The Learning Bar Inc.

Willms, J. D., Durepos, D., López García, A. Y., Laurie, R., \& Tramonte, L. (2015). Design and composition of contextual questionnaires for the PISA for development study. Fredericton, NB: The Learning Bar Inc. 
Manuscrito 3

\author{
AVALIAÇÃO DA IGUALDADE E EQUIDADE \\ DO SISTEMA EDUCACIONAL PÚBLICO BRASILEIRO
}

\begin{abstract}
Resumo
Pautadas pelos princípios de equidade, as políticas educacionais precisam buscar superar as diferenças sociais e étnicas, a fim de prover igualdade de oportunidade. Contudo, na prática, o sistema educacional parece reproduzir desigualdades sociais. De modo a analisar a igualdade e equidade do sistema educacional brasileiro, este estudo tem como objetivo verificar diferenças de desempenho acadêmico e diferenças de acesso a recursos escolares conforme a condição socioeconômica do estudante. O estudo foi realizado utilizando as informações de 862.114 alunos do $9^{\circ}$ ano do ensino fundamental, provindos de 14.730 escolas, participantes da Prova Brasil 2009. Os resultados apontaram que a chance de sucesso acadêmico de um estudante pobre é de aproximadamente um terço da chance de um estudante de outro nível socioeconômico; além disso, o estudante pobre tem menos acesso a recursos escolares. Análises por dependência administrativa mostraram que as redes de ensino fazem diferença na promoção da igualdade e equidade. De modo geral, os resultados chamam a atenção para a vulnerabilidade a que estão expostos os estudantes pobres no sistema educacional brasileiro, fazendo-se urgente a reflexão acerca de políticas compensatórias.
\end{abstract}

Palavras-chave: equidade e igualdade; desempenho acadêmico; recursos escolares. 


\begin{abstract}
Guided by the principles of equity, educational policies must seek to overcome social and ethnic differences in order to provide equality of opportunity. However, in practice, the educational system seems to reproduce social inequalities. In order to analyze the equality and equity of the Brazilian educational system, this study aims to verify differences in academic performance and differences in access to school resources according to student socioeconomic level. The study was conducted using the information of 862,114 students in the 9th grade of elementary school, stemmed from 14,730 schools, participants of Prova Brasil 2009. The results showed that the odds of academic success of a poor student is approximately one third the odds of a student of other socioeconomic level; moreover, the poor student has less access to school resources. Analysis by administrative subordination showed that school systems make a difference in promoting equality and equity. Overall, the results point to the vulnerability that poor students are exposed in Brazilian educational system, making it urgent to reflect on compensatory policies.
\end{abstract}

Keywords: equity and equality; academic performance; school resources. 
A disseminação de ideologias igualitárias e a expansão dos sistemas educacionais surgem após a Segunda Guerra Mundial, calcadas nos pressupostos otimistas de que a expansão dos sistemas educacionais é a chave para a promoção da igualdade de oportunidades (Karabek \& Halsey apud Koslinski \& Alves, 2012). Hoje, após quase 70 anos, podemos afirmar que o sistema educacional brasileiro tem provido igualdade de oportunidades? Ou será a escola uma mera reprodutora da estrutura da relação entre as classes sociais?

A educação carrega o estigma da emancipação. Há esperança de que por meio dela seja possível proporcionar mudanças que contemplem a inclusão e a cidadania. Como diz Santiago (2012):

ao acolher todas as crianças e jovens de uma geração com a responsabilidade de formar os cidadãos que constituem o futuro de uma nação, a escola, indubitavelmente, acolhe a esperança de um mundo melhor e torna-se uma instituição importante e indispensável na luta por uma sociedade mais equânime, com menos sofrimento e menos riscos ecológicos (p.4).

Nesse sentido, a educação precisa exercer um papel de política compensatória das desigualdades. A diferenciação dos princípios de igualdade e equidade encontra suporte com os estudos relativos à justiça distributiva na psicologia social. De acordo com o princípio de igualdade, o justo é a divisão igualitária de recursos de modo que os participantes recebam a mesma quantidade independentemente de condições de esforço, capacidade ou necessidade. Já o princípio de equidade determina que a alocação de recursos seja proporcional, baseada no grau de necessidade (Aams, 1965; Leventhal, Popp, \& Sawyer, 1973).

Estendendo tais conceitos para a educação, o Estado, conforme prevê a Constituição Federal Brasileira, deve garantir o direito à educação a todos, sem distinção de renda, etnia, região ou qualquer outra característica. Portanto, a garantia desse direito não pode ser exclusiva de um determinado grupo social. Para isso, disparidades sociais não podem se 
refletir em desigualdades educacionais. As políticas de equidade devem implicar a satisfação das necessidades básicas da população, priorizando-as segundo seus graus de urgência relativa, privilegiando a solução do problema da maioria (Cohen \& Franco, 2013). Portanto, a educação, pautada em políticas regidas pelo princípio da equidade, precisa buscar superar as desigualdades, em busca da qualidade para todos.

No contexto de avaliação de sistemas educacionais, Willms, Tramonte, Duarte e Bos (2012) defendem que o termo igualdade deve ser utilizado quando se analisam as diferenças nos resultados educacionais entre subpopulações. Por exemplo, um sistema educacional é dito igualitário quando estudantes de diferentes grupos em termos do seu nível socioeconômico, raça ou gênero não apresentam distribuições diferentes de desempenho acadêmico. A ausência de diferenças no desempenho é indicativo de que o direito a uma educação de qualidade está garantido a todos. Ou seja, não há privilégio ou benefício a um determinado grupo, o sistema educacional é capaz de fornecer uma educação igualitária para estudantes de subpopulações distintas.

Considerando os diferentes contextos em que os estudantes estão inseridos, a garantia da igualdade perpassa políticas de equidade na alocação de recursos. Logo, Willms, Tramonte, Duarte e Bos (2012) usam o termo equidade para se referir ao acesso a recursos e processos escolares que afetam o desempenho acadêmico. Um sistema equânime é aquele que proporciona oportunidades equivalentes de aprendizagem para os seus estudantes. A Figura 1 apresenta esquematicamente a aplicação dos termos equidade e igualdade proposta por Willms, Tramonte, Duarte e Bos (2012). 


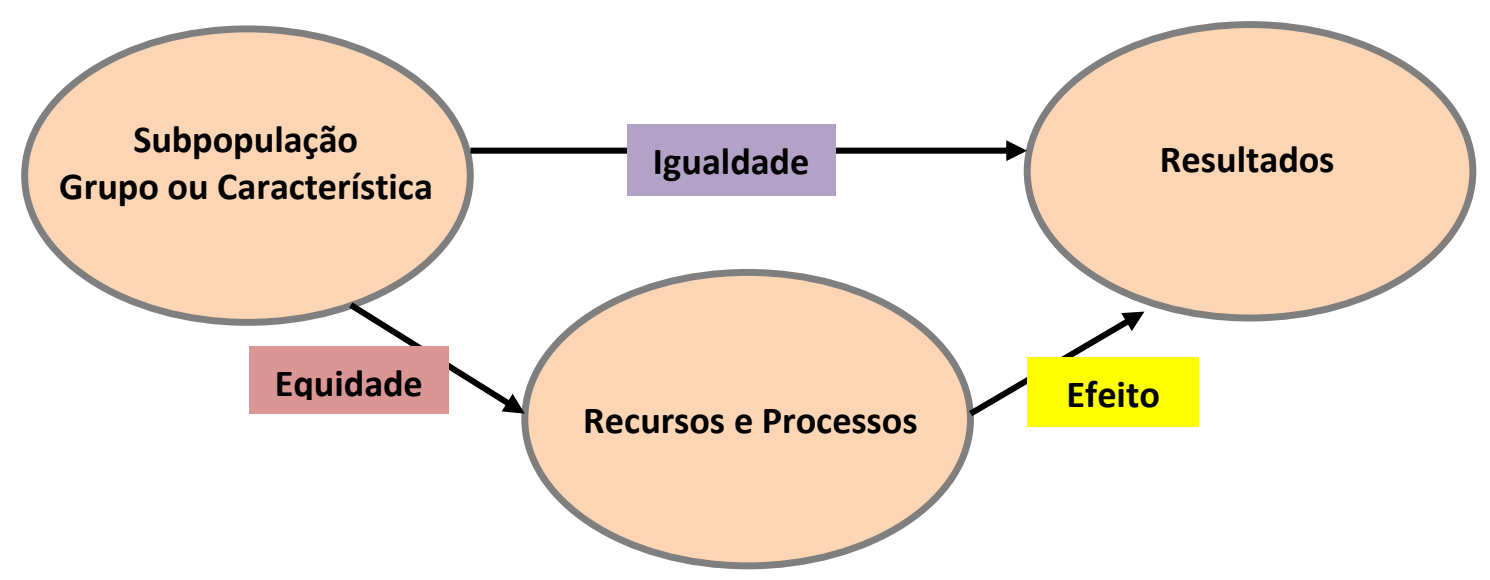

Figura 1.

Modelo de avaliação de igualdade e equidade de um sistema educacional (Adaptado de Willms, Tramonte, Duarte, \& Bos, 2012).

Os estudos sobre igualdade e equidade são um dos eixos de pesquisa dentro da área de eficácia escolar. Inclusive, é possível afirmar que as duas dimensões gerais da eficácia escolar são a qualidade e a equidade. Há uma tendência nos estudos comparativos internacionais (como Programme for International Student Assessment - PISA, Trends in International Mathematics and Science Study - TIMSS, Progress in Reading Literacy Study - PIRLS, etc.) em relatar a eficácia de sistemas educacionais em termos dessas duas dimensões (Reynolds et. al. 2014). A eficácia escolar, ao englobar qualidade e equidade, compreende o quanto a escola tem desempenho melhor do que outras e o quanto ela é capaz de compensar diferenças de entrada (Reynolds, Sammons, De Fraine, Van Damme, Townsend, Teddlie, \& Stringfield, 2014).

As diferenças de desempenho entre gênero, etnia e nível socioeconômico têm sido bastante investigadas nos estudos sobre equidade. Alguns pesquisadores apontam que essas diferenças estão relacionadas ao fato de os estudantes negros e pobres terem acesso a escolas de pior qualidade (Fryer \& Levitt, 2004). Já outros estudos sugerem que a qualidade da escola não é a questão (Bali \& Alvarez, 2004). Num estudo realizado por 
Strand (2010), verificou-se a existência do que o autor denominou de equidade na eficácia, uma vez que as escolas mais eficazes para os brancos são também mais eficazes para os alunos negros. No entanto, as diferenças de desempenho entre os grupos tendem a aumentar.

As pesquisas brasileiras sobre igualdade e equidade também estão focadas nas diferenças de gênero, etnia e nível socioeconômico (Andrade, Franco, \& Carvalho, 2003; Soares, 2004a; Soares, 2004b; Soares \& Alves, 2003; Soares \& Andrade, 2006). Essas pesquisas concentram-se em investigar os fatores escolares relacionados à escola eficaz que podem contribuir para diminuir as desigualdades. Por exemplo, no estudo de Soares e Alves (2003), investiga-se se melhor qualificação docente, melhores salários e melhores equipamentos têm efeito significativo no sentido de diminuir as diferenças de desempenho entre alunos brancos e negros. Os resultados encontrados, no entanto, mostram que a melhoria nas condições escolares acarreta aumento das diferenças.

No estudo de Soares e Andrade (2006) verificou-se que as escolas estaduais, municipais e particulares não apresentam efeito na relação entre nível socioeconômico e desempenho. Já as escolas federais tendem a acirrar as diferenças de desempenho provocadas pelo nível socioeconômico.

Outros estudos buscaram verificar o quanto processos associados ao professor e à escola poderiam contribuir para diminuir as diferenças de desempenho por sexo, raça, nível socioeconômico e atraso escolar (Soares, 2004b). Novamente, a maior parte das variáveis tende a amplificar o efeito das variáveis no sentido de aumentar as diferenças entre os grupos. Já as poucas variáveis que parecem diminuir as diferenças não apresentam aparentemente razoabilidade teórica. Por exemplo, no estudo de Soares (2004b), o aumento da segurança na escola parece diminuir o efeito do atraso escolar sobre o desempenho. 
Inicialmente, não é aparente uma explicação para segurança exercer influência sobre o efeito de atraso escolar.

De modo geral, os artigos sobre igualdade e equidade mostram que os fatores produtores de eficácia escolar têm acirrado a diferenças entre grupos de estudantes. Para Soares (2004a), ao promover melhorarias no ambiente escolar, os alunos mais favorecidos tendem a usufruir mais do que os alunos menos favorecidos. Esses resultados são preocupantes na medida em que indicam que os fatores que contribuem para um melhor resultado estão associados a um aumento das desigualdades.

Albanez, Ferreira e Franco (2002) também encontraram resultados semelhantes ao verificar que o nível de escolaridade do professor faz com que a escola seja mais eficaz, mas torna a escola mais desigual, aumentando a importância do nível socioeconômico do aluno para o seu desempenho. Apesar de os resultados não serem os ideais em termos de igualdade e equidade, os autores ressaltam a importância de se continuar a investir na melhoria da escola, uma vez que o benefício do nível de escolaridade do professor sobre o desempenho ocorre para todos os alunos. A diferença é que o benefício ocorre de forma mais efetiva para os alunos de nível socioeconômico mais elevado.

A área tem avançado muito com a realização de estudos multinível que buscam compreender efeitos diferenciados da escola. Willms, Tramonte, Duarte e Bos (2012) chama a atenção, no entanto, para a necessidade de também nos preocuparmos em como fazer chegar os resultados a educadores e gestores. Nesse sentido, análises baseadas em variáveis categóricas tendem a ser mais transparentes, e são mais fáceis para a definição de metas.

As pesquisas brasileiras têm focado em compreender a interação entre níveis, buscando identificar variáveis do nível da escola que diminuem a interferência de variáveis do nível do aluno. Todavia, há uma carência de estudos que demonstrem as inequidades do 
país. É preciso investigar como os recursos escolares estão distribuídos e identificar os grupos de estudantes que têm menos acesso aos investimentos escolares.

Assim, além de se estudar as variáveis moderadoras que possibilitam potencializar ou minimizar o efeito de gênero, raça e nível socioeconômico sobre o desempenho, é preciso compreender as inequidades que existem no país e o quanto elas tornam alguns grupos mais vulneráveis. O real diagnóstico dos grupos mais vulneráveis e de que forma eles estão vulneráveis é essencial para uma atuação diferenciada por parte de gestores e professores.

Como apontado pelo movimento Todos pela Educação (2011), para avançar nas políticas educacionais para a promoção da equidade e da inclusão, é necessária maior precisão sobre os dados da realidade brasileira para que as ações possam ser mais eficazes. Com isso, verifica-se a necessidade de estudos que busquem identificar e mensurar as desigualdade e inequidades, bem como demonstre o quanto alguns grupos têm relativamente poucas chances de sucesso escolar ao menos que intervenções sejam realizadas.

Este estudo tem como objetivo verificar as diferenças de desempenho por nível socioeconômico e demonstrar os problemas de equidade a que estão expostos os estudantes pobres do sistema educacional brasileiro. Para tanto, tem-se como objetivos específicos:

1. Verificar as diferenças de desempenho dos estudantes em matemática por nível socioeconômico (igualdade).

2. Estimar as chances de a população pobre alcançar o nível adequado de proficiência (igualdade).

3. Estimar as chances de a população pobre ter acesso a recursos educacionais (equidade). 
A escolha por analisar as diferenças por nível socioeconômico foi realizada porque a pobreza ainda é um sério problema no contexto brasileiro, sendo a educação a esperança para uma melhoria do país (Barros, Henrique, \& Mendonça, 2000). Para avaliação da igualdade serão utilizados como referência os resultados da Prova Brasil, avaliação externa realizada pelo governo federal e utilizada como uma das formas de monitorar a garantia do direito de aprender. Já a análise de equidade perpassa a definição de quais seriam os recursos essenciais para a garantia da qualidade de ensino. Para isso, será utilizado como referência o modelo de prosperidade educacional de Willms (2015).

O modelo de prosperidade educacional, proposto por Willms (2015), tem como objetivo estabelecer os elementos essenciais de uma escola que, ao serem garantidos, sinalizam o sucesso de um sistema educacional. O termo "prosperidade educacional" tem conotação com o termo "prosperidade econômica", geralmente utilizado quando os indicadores econômicos apontam crescimento.

O modelo define que os fundamentos do sucesso educacional estão na família, na escola e na comunidade. Tendo como foco a escola, os elementos essenciais (pilares) centram-se no processo de aprendizagem e em aspectos que podem ser modificados pelas escolas. Esses pilares podem se diferenciar conforme a etapa de ensino. Para os estudantes no final do ensino fundamental, quatro pilares são definidos: (1) qualidade de ensino; (2) ambiente inclusivo; (3) tempo de aprendizado; e (4) recursos escolares.

A definição desses pilares teve como base os vastos estudos na área de eficácia escolar (Willms, Durepos, López García, Laurie, \& Tramonte, 2015). Medidas para representar esses pilares tendo como base os questionários da Prova Brasil já foram desenvolvidas por Karino (2016). Essas medidas serão utilizadas neste estudo. 


\section{Método}

\section{Participantes}

Este estudo foi realizado a partir dos microdados da Avaliação Nacional do Rendimento Escolar de 2009 (ANRESC - Prova Brasil) disponibilizados pelo Instituto Nacional de Estudos e Pesquisas Educacionais Anísio Teixeira (Inep). Essa avaliação externa foi criada em 2005 com o objetivo de avaliar de forma censitária o rendimento escolar de todas as escolas públicas do ensino fundamental no Brasil.

Especificamente, foram selecionados os alunos do $9^{\circ}$ ano do ensino fundamental que responderam à prova de Matemática. A base de dados final foi composta por 1.909.331 alunos, provindos de 31.944 escolas.

\section{Instrumentos/Variáveis}

Os instrumentos e variáveis utilizados nesse estudo foram:

- Prova de Matemática: teste cognitivo de avaliação do desempenho dos alunos em Matemática desenvolvido pelo Inep para compor a Prova Brasil. O cálculo das proficiências dos alunos tem como base a teoria de resposta ao item, o que possibilita a comparabilidade entre anos e entre série, numa escala com média 250 e desviopadrão 50. Com base na proficiência dos estudantes em matemática foi desenvolvido um indicador de sucesso escolar. Considerou-se sucesso escolar o desempenho em matemática superior a 300, valor atribuído como nível adequado de proficiência (Fernandes, 2007; INEP, 2007).

- Questionários contextuais dos alunos, professores, diretores e escolas: esses instrumentos buscam acessar informações acerca do nível socioeconômico e cultural da população, além de abordar aspectos familiares e escolares. A partir desses questionários, no estudo de Karino (2016), foram sugeridas e construídas medidas 
para representar os pilares do modelo de prosperidade educacional. Este estudo utiliza as medidas propostas para três dos quatro pilares (qualidade da educação; tempo de aprendizado e recursos da escola), além da medida de nível socioeconômico. O quarto pilar, ambiente inclusivo, não foi considerado porque, conforme Karino (2016), os questionários da Prova Brasil não possuem medidas representativas desse construto. Para a avaliação de risco, as medidas foram tratadas como dicotômicas de acordo com a descrição apresentada na Tabela 1.

- Questionário do Censo da Educação Básica: o Censo Escolar é um levantamento de dados referentes à escola, realizado anualmente pelo Inep. A partir dos dados do Censo 2009 foi construído o Indicador de Infraestrutura Escolar (Soares Neto, Jesus, Karino, \& Andrade, 2013). Esse indicador também foi utilizado como dicotômico nas análises (Tabela 1).

Tabela 1

Descrição das variáveis utilizadas no estudo

\begin{tabular}{|c|c|c|}
\hline Variável & Instrumento & Codificação \\
\hline $\begin{array}{l}\text { Escala de proficiência em } \\
\text { matemática }\end{array}$ & $\begin{array}{l}\text { Prova de Matemática } \\
\text { (Prova Brasil) }\end{array}$ & Sem codificação. \\
\hline Sucesso Escolar & $\begin{array}{l}\text { Prova de Matemática } \\
\text { (Prova Brasil) }\end{array}$ & $\begin{array}{l}1 \text { = proficiência em matemática igual ou } \\
\text { superior a } 300 \text { (nível adequado). } \\
0=\text { proficiência em matemática menor que } 300 \text {. }\end{array}$ \\
\hline $\begin{array}{l}\text { Professor corrige dever de } \\
\text { casa (pilar qualidade do } \\
\text { ensino) }\end{array}$ & do aluno & $\begin{array}{l}1=\text { sempre ou quase sempre } \\
0=\text { frequência menor que sempre ou quase } \\
\text { sempre }\end{array}$ \\
\hline $\begin{array}{l}\text { Adequação do currículo } \\
\text { (pilar qualidade do ensino) }\end{array}$ & Quest. do professor & $\begin{array}{l}1=\text { mais de } 80 \% \text { dos professores da escola } \\
\text { avaliam o currículo como adequado. } \\
0=\text { menos de } 80 \% \text { dos professores da escola } \\
\text { avaliam o currículo como adequado. }\end{array}$ \\
\hline $\begin{array}{l}\% \text { de cumprimento do } \\
\text { currículo (pilar qualidade } \\
\text { do ensino) }\end{array}$ & Quest. do professor & $\begin{array}{l}1=\text { mais de } 80 \% \text { do currículo cumprido. } \\
0=\text { menos de } 80 \% \text { do currículo cumprido. }\end{array}$ \\
\hline $\begin{array}{l}\text { Escolaridade do professor } \\
\text { (pilar qualidade do ensino) }\end{array}$ & Quest. do professor & $\begin{array}{l}1=\text { escolaridade mínima de nível superior } \\
\text { completo. } \\
0=\text { escolaridade inferior a nível superior } \\
\text { completo. }\end{array}$ \\
\hline $\begin{array}{l}\text { Escolaridade do diretor } \\
\text { (pilar qualidade do ensino) }\end{array}$ & Quest. do diretor & $\begin{array}{l}1 \text { = escolaridade mínima de nível superior } \\
\text { completo. } \\
0=\text { escolaridade inferior a nível superior } \\
\text { completo. }\end{array}$ \\
\hline
\end{tabular}




\begin{tabular}{|c|c|c|}
\hline $\begin{array}{l}\text { Ter material didático (pilar } \\
\text { qualidade do ensino) }\end{array}$ & Quest. do professor & $\begin{array}{l}1 \text { = escolas em que todos os estudantes têm } \\
\text { material didático. } \\
0=\text { escolas em que nem todos os estudantes têm } \\
\text { material didático. }\end{array}$ \\
\hline $\begin{array}{l}\text { Indisciplina (pilar tempo } \\
\text { de aprendizado) }\end{array}$ & Quest. do professor & $\begin{array}{l}1=\text { mais de } 90 \% \text { dos professores avaliam a } \\
\text { indisciplina como sendo um problema na escola. } \\
0=\text { menos de } 90 \% \text { dos professores avaliam a } \\
\text { indisciplina como sendo um problema na escola. }\end{array}$ \\
\hline $\begin{array}{l}\text { Alunos faltam às aulas } \\
\text { (pilar tempo } \\
\text { aprendizado) }\end{array}$ & Quest. do professor & $\begin{array}{l}1=\text { pelo menos um professor avalia que alunos } \\
\text { faltando aulas é problema na escola. } \\
0=\text { nenhum professor considera que alunos } \\
\text { faltando aulas é problema na escola. }\end{array}$ \\
\hline $\begin{array}{l}\text { Atividades de tutoria (pilar } \\
\text { tempo de aprendizado) }\end{array}$ & do diretor & $\begin{array}{l}1=\text { escola oferece atividades de tutoria. } \\
0=\text { escola não oferece atividades de tutoria. }\end{array}$ \\
\hline $\begin{array}{l}\text { Escala de condições } \text { de } \\
\text { infraestrutura (pilar de } \\
\text { recursos da escola) }\end{array}$ & Quest. da escola & $\begin{array}{l}1=\text { escola apresenta boas condições de } \\
\text { infraestrutura em todos os elementos avaliados } \\
\text { (nível } 4 \text { da escala) } \\
0=\text { níveis inferiores a } 4 .\end{array}$ \\
\hline $\begin{array}{lll}\text { Escala } & \text { de } & \text { equipamentos } \\
\text { (pilar de } & \text { recursos da } \\
\text { escola) } & & \end{array}$ & Quest. da escola & $\begin{array}{l}1 \text { = escola possui equipamentos como máquina } \\
\text { copiadora e de prevenção contra incêndios } \\
\text { (níveis } 3 \text { e } 4 \text { da escala } 2 \text { ) } \\
0=\text { níveis inferiores a } 3 .\end{array}$ \\
\hline Nível socioeconômico & Quest. do aluno & $\begin{array}{l}\text { Sem codificação. Utilizou-se os cinco níveis da } \\
\text { escala original. }\end{array}$ \\
\hline Pobreza & Quest. do aluno & $\begin{array}{l}1 \text { = nível } 1 \text { da escala de nível socioeconômico. } \\
0=\text { nível socioeconômico superior a } 1\end{array}$ \\
\hline Escala de infraestrutura & $\begin{array}{l}\text { Censo da Educação } \\
\text { Básica }\end{array}$ & $\begin{array}{l}1=\text { escolas possuem infraestrutura adequada ou } \\
\text { avançada (nível igual ou superior a } 5 \text { ). } \\
0=\text { níveis inferior a } 5 .\end{array}$ \\
\hline
\end{tabular}

\section{Procedimentos de análise de dados}

A primeira etapa das análises consistiu em realizar o tratamento da base de dados, agrupando as medidas provindas de diferentes fontes. Foi realizada análise exploratória dos dados e foram mantidos somente os estudantes com mais $70 \%$ do questionário respondido, o que ocasionou uma redução de $4 \%$ da base de dados. Não foi realizado tratamento dos dados missing, sendo consideradas, em cada etapa de análise, todas as respostas válidas.

A segunda etapa das análises objetivou verificar a igualdade e a equidade do sistema educacional brasileiro. A igualdade foi verificada com base na análise de diferenças entre a média de desempenho dos estudantes por nível socioeconômico (ANOVA e comparações múltiplas - Teste de Tukey e Scheffea), na análise das chances de um estudante pobre alcançar o nível adequado de proficiência em matemática e na análise da relação entre 
nível socioeconômico e desempenho (regressão). Para a segunda análise, especificamente, três indicadores foram calculados: (1) o percentual de estudantes pobres e nos demais níveis socioeconômicos que conseguem atingir o nível adequado; (2) a razão entre o percentual de estudantes pobres no nível adequado em relação ao percentual de estudantes de outro nível socioeconômico no nível adequado, o que indica o sucesso relativo de um grupo em relação ao outro; e (3) a razão de chance (odds-ratio) de sucesso de um estudante pobre em relação a um de outro nível socioeconômico.

A análise de equidade teve como foco verificar a chance de um estudante pobre ter acesso a recursos escolares ditos como essenciais no modelo de prosperidade educacional. As análises de chance tiveram como base os três indicadores já descritos.

As análises de igualdade e equidade foram reproduzidas também por unidade da federação/rede. Essa diferenciação é interessante dado que ela considera a divisão administrativa do sistema educacional brasileiro. Por ser uma rede muito pequena, não foram realizadas análises para a rede Federal.

\section{Resultados}

A análise de variância apontou diferenças significativas $(\mathrm{p}<0,01)$ entre as médias de desempenho em matemática dentre os cinco níveis socioeconômicos investigados. Em seguida, procedeu-se a um teste de comparação múltipla das médias. Nessa análise, verificou-se diferença significativa entre todas as médias por nível socioeconômico $(\mathrm{p}<$ 0,01). O tamanho de efeito ( $d$ de Cohen) das diferenças entre as médias foi de 0,30 (entre grupos 1 e 2), diminuindo gradualmente até 0,02 (entre grupos 4 e 5). Quando se compara a média dos grupos 1 e 5, o tamanho de efeito foi de 0,66. A Tabela 2 apresenta as médias de desempenho por nível socioeconômico. 
Tabela 2

Média e desvio-padrão do desempenho em matemática por nível socioeconômico

\begin{tabular}{|c|c|c|c|c|}
\hline Nível & $\begin{array}{l}\text { Intervalo de } \\
\text { Proficiência }\end{array}$ & $\begin{array}{c}\text { Interpretação } \\
\text { Escala de Nível Socioeconômico }\end{array}$ & $\begin{array}{l}\text { Proficiência } \\
\text { Média em } \\
\text { Matemática }\end{array}$ & DP \\
\hline 1 & $\leq-0,5$ & $\begin{array}{l}\text { Neste nível, os estudantes provavelmente } \\
\text { possuem em suas casas uma TV, uma geladeira, } \\
\text { um banheiro, um rádio e um aparelho DVD. }\end{array}$ & 228,4 & 41,1 \\
\hline 2 & $\leq 0,5$ & $\begin{array}{l}\text { Neste nível, os estudantes provavelmente } \\
\text { possuem em suas casas uma TV, uma geladeira, } \\
\text { um banheiro, um radio, um aparelho DVD e } \\
\text { adicionalmente uma máquina de lavar roupa e a } \\
\text { mãe concluiu o ensino fundamental. }\end{array}$ & 241,3 & 44,1 \\
\hline 3 & $\leq 1,0$ & $\begin{array}{l}\text { Neste nível, os estudantes provavelmente } \\
\text { começam a ter um suas casas duas TVs, um } \\
\text { computador e o pai também concluiu o ensino } \\
\text { fundamental. }\end{array}$ & 251,3 & 46,3 \\
\hline 4 & $\leq 2,5$ & $\begin{array}{l}\text { Neste nível, os estudantes provavelmente } \\
\text { começam a ter um suas casas dois rádios, três ou } \\
\text { mais TVs, um carro e dois banheiros. }\end{array}$ & 258,4 & 48,1 \\
\hline 5 & $>2,5$ & $\begin{array}{l}\text { Neste nível, os estudantes provavelmente } \\
\text { começam a ter um suas casas três ou mais rádios, } \\
\text { dois ou mais carros, três ou mais banheiros, duas } \\
\text { ou mais geladeiras, um freezer, uma empregada } \\
\text { doméstica e os pais completaram pelo menos o } \\
\text { ensino médio. }\end{array}$ & 259,7 & 53,1 \\
\hline
\end{tabular}

Os resultados indicam, portanto, desigualdades no desempenho em matemática conforme o nível socioeconômico dos estudantes. Dado que existe desigualdade, em seguida, buscou-se analisar o que isso representa em termos de chances de sucesso de um estudante pobre no Brasil. Chama a atenção o percentual baixo de estudantes que alcançam o nível adequado de proficiência em matemática no Brasil, cerca de 5\% $(0,052)$ no grupo pobre e $13 \%(0,129)$ nos demais grupos (Tabela 3$)$. Isso significa que um sucesso relativo de $40 \%(0,052 / 0,129)$. Em termos de razão de chance, podemos afirmar que a chance de sucesso (nível adequado de proficiência) de um aluno pobre é $37 \%$ a chance de sucesso de um estudante pertencente a um grupo socioeconômico diferente (Razão de chance = $((0,052 / 0,948) /(0,129 / 0,871))$. 
Ainda na Tabela 3 são destacados alguns resultados de Unidade da Federação/rede. As redes estaduais de Minas Gerais e do Rio Grande do Sul são as que apresentam maior percentual de alunos que atingem o nível de adequado de proficiência. Por outro lado, as redes municipal e estadual do Amapá apresentam os menores percentuais de alunos com o nível adequado em matemática em 2009.

As redes com maior desigualdade entre pobres e demais grupos socioeconômicos no Brasil são: municipal do Amapá, estadual de Roraima e municipal de Tocantins. Nessas redes, a chance de um estudante pobre ter sucesso escolar (nível adequado) é cerca de um terço da chance de um estudante de outro nível socioeconômico. As redes com maior igualdade entre pobres e demais grupos socioeconômicos no Brasil são: municipal de Rondônia, estadual do Amazonas e estadual do Rio de Janeiro. Nessas redes, a chance de um estudante pobre ter sucesso escolar (nível adequado) é mais próxima à dos estudantes dos demais níveis socioeconômicos. No entanto, os percentuais de alunos com sucesso escolar são baixos para ambos os grupos. Os resultados de todas as UF/redes são apresentados no Anexo 1. 
Percentual de estudantes que atingem o nível adequado de proficiência, sucesso relativo e razão de chance de sucesso

\begin{tabular}{|c|c|c|c|c|c|c|c|}
\hline \multirow[b]{2}{*}{ UF/Rede } & \multirow[b]{2}{*}{$\begin{array}{l}\mathrm{N} \text { (alunos } \\
\text { na rede) }\end{array}$} & \multicolumn{2}{|c|}{ Grupo pobre } & \multicolumn{2}{|c|}{ Demais grupos } & \multicolumn{2}{|c|}{$\begin{array}{c}\text { Igualdade } \\
\text { (Pobres x Outros) }\end{array}$} \\
\hline & & $\begin{array}{l}\% \text { Abaixo } \\
\text { adequado }\end{array}$ & $\begin{array}{c}\% \\
\text { Sucesso }\end{array}$ & $\begin{array}{l}\% \text { Abaixo } \\
\text { adequado }\end{array}$ & $\begin{array}{c}\% \\
\text { Sucesso }\end{array}$ & $\begin{array}{l}\text { Sucesso } \\
\text { relativo }\end{array}$ & $\begin{array}{c}\text { Razão de } \\
\text { chance de } \\
\text { sucesso }\end{array}$ \\
\hline Brasil & 1.908 .395 & 95 & 05 & 87 & 13 & 0,40 & 0,37 \\
\hline MG/Estadual & 159.862 & 88 & 12 & 78 & 22 & 0,56 & 0,50 \\
\hline RS/Estadual & 55.244 & 90 & 10 & 82 & 18 & 0,53 & 0,48 \\
\hline AP/Estadual & 6.718 & 98 & 02 & 96 & 04 & 0,45 & 0,44 \\
\hline AP/Municipal & 628 & 99 & 01 & 96 & 04 & 0,30 & 0,29 \\
\hline RR/Estadual & 4.605 & 97 & 03 & 93 & 07 & 0,37 & 0,35 \\
\hline TO/Municipal & 2.919 & 95 & 05 & 86 & 14 & 0,37 & 0,33 \\
\hline RO/Municipal & 4.629 & 95 & 05 & 93 & 07 & 0,72 & 0,70 \\
\hline AM/Estadual & 29.239 & 93 & 07 & 91 & 09 & 0,74 & 0,72 \\
\hline RJ/Estadual & 49.522 & 94 & 06 & 91 & 09 & 0,67 & 0,65 \\
\hline
\end{tabular}

Outra possibilidade de avaliação da igualdade por nível socioeconômico é por meio da inclinação ou gradiente de regressão. Willms (2006) sugere a utilização desse gradiente para descrever a relação entre o desempenho dos estudantes e a medida do nível socioeconômico nas análises realizadas para o PIRLS e para o PISA. Numa regressão, o valor de inclinação (slope) indica o nível de igualdade. Quanto maior a inclinação, menor a igualdade. Outro importante valor é o $\mathrm{R}^{2}$, o qual indica a proporção de variância explicada pelo nível socioeconômico. Quanto maior o valor de $\mathrm{R}^{2}$, mais forte é a relação entre desempenho e nível socioeconômico (Willms, 2010). Essa análise foi realizada no nível da escola. Assim, foi considerada a média de desempenho em matemática da escola e a média do nível socioeconômico dos alunos que compõem a escola.

Os resultados da análise de regressão para o Brasil foram slope $=22,6$ e $\mathrm{R}^{2}=0,39$, o que indica haver relação positiva entre nível socioeconômico e desempenho. A Figura 2 mostra essa relação. Nota-se que na medida em que o nível socioeconômico das escolas aumenta, tende a aumentar o desempenho médio em matemática. Quando se realiza a 
análise por UF/rede, notam-se diferenças grandes, o que mostra que o sistema educacional brasileiro é bastante heterogêneo. A Figura 2 mostra um exemplo de UF/rede bastante igualitários e outro, com maior desigualdade. Os valores do gradiente de inclinação e de $\mathrm{R}^{2}$ de todas as UF/Redes são apresentados no Anexo 1.
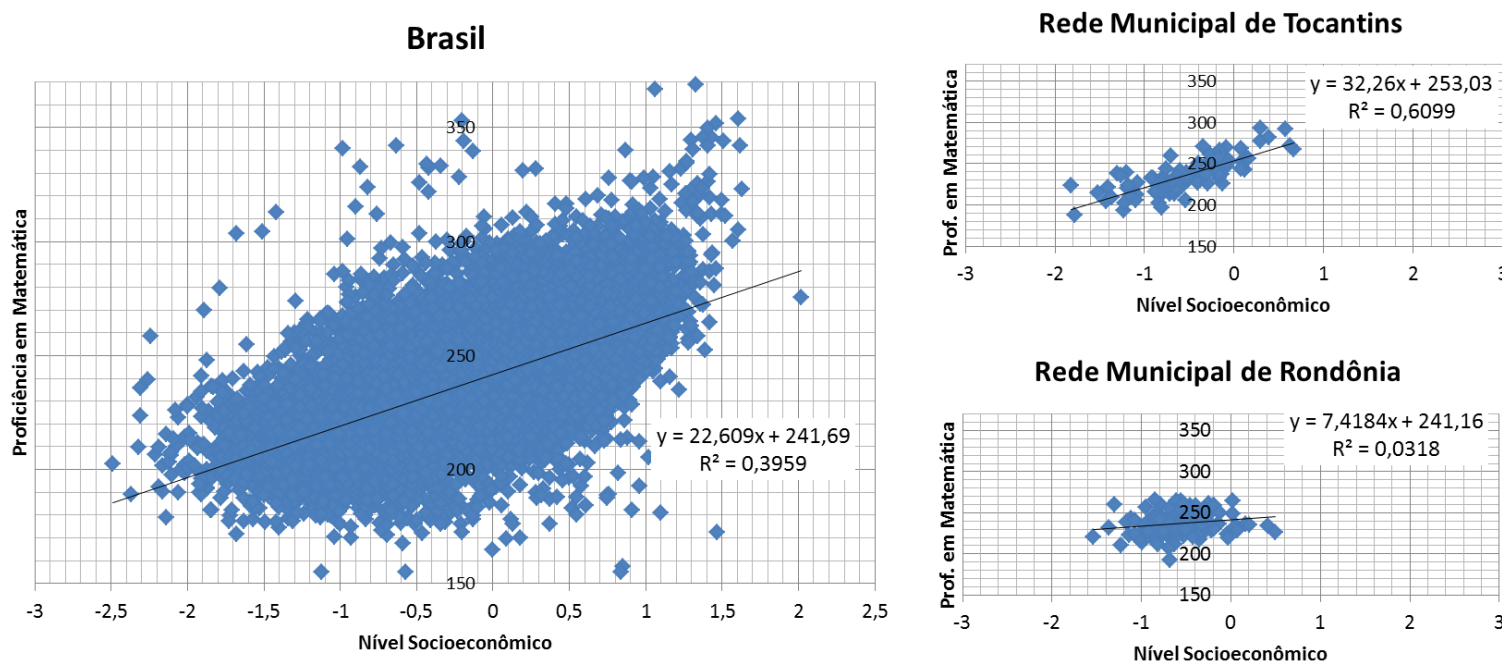

Rede Municipal de Rondônia

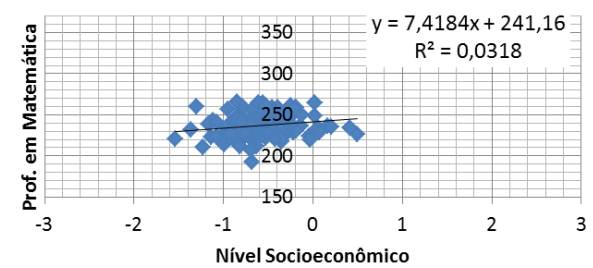

Figura 2

Gráfico de regressão: nível socioeconômico e desempenho em matemática da escola

A Tabela 4 apresenta os resultados das análises de equidade. Em todos os pilares avaliados, percebe-se que o sistema educacional brasileiro ainda enfrenta dificuldades de prover acesso a elementos básicos como professor com formação adequada e infraestrutura escolar mínima. Além disso, ainda se enfrentam problemas como indisciplina e alunos faltando às aulas. Especificamente no pilar qualidade do ensino, ressalta-se que somente $13 \%$ das escolas no Brasil conseguem cumprir com pelo menos $80 \%$ do currículo. 
Tabela 4

Percentual de estudantes com acesso a recursos escolares, chance relativa e razão de chance de acesso

\begin{tabular}{lccccc}
\hline \multirow{2}{*}{ Recurso/ Processo } & \multicolumn{3}{c}{ Percentual de acesso } & \multicolumn{2}{c}{ Equidade (Pobres x Outros) } \\
\cline { 2 - 6 } & Brasil & $\begin{array}{c}\text { Grupo } \\
\text { pobre }\end{array}$ & $\begin{array}{c}\text { Demais } \\
\text { grupos }\end{array}$ & $\begin{array}{c}\text { Acesso } \\
\text { relativo }\end{array}$ & $\begin{array}{c}\text { Razão de chance } \\
\text { de acesso }\end{array}$ \\
\hline Professor corrige dever de casa & 30 & 33 & 29 & 1,12 & 1,17 \\
Adequação do currículo & 62 & 62 & 63 & 0,99 & 0,96 \\
\% cumprimento do currículo & 13 & 10 & 14 & 0,71 & 0,68 \\
Escolaridade professor & 29 & 27 & 30 & 0,90 & 0,86 \\
Escolaridade diretor & 72 & 66 & 74 & 0,89 & 0,69 \\
Ter material didático & 47 & 43 & 49 & 0,88 & 0,79 \\
\hline Indisciplina & Pilar 3: Tempo de Aprendizado & & 0,87 \\
Alunos faltam às aulas & 50 & 48 & 51 & 0,93 & 1,07 \\
Atividades de tutoria & 69 & 70 & 69 & 1,02 & 0,49 \\
\hline
\end{tabular}

Ao se analisar os resultados de equidade, verifica-se que a chance de um professor corrigir o dever de casa e de os alunos faltarem às aulas é maior entre os estudantes pobres. A maior abstenção dos alunos pobres está relacionada com o contexto social mais precário, que os impede muitas vezes de ir à escola. Já o fato desses estudantes apresentarem desempenhos mais baixos possivelmente tem relação com a tendência de o professor corrigir mais o dever de casa. O recurso que possui maior equidade é adequação do currículo. Esse resultado era esperado porque o currículo adotado por uma secretaria de ensino é igual para todas as escolas, independente do nível socioeconômico das mesmas.

Contudo, nota-se que os alunos pobres têm uma chance menor de acesso à maioria dos recursos analisados. A maior diferença de acesso está em equipamentos. A razão de chance é 0,28 , o que indica que a chance de um estudante pobre ter acesso a equipamentos é $28 \%$ a chance de um estudante de outro nível socioeconômico. A menor oportunidade de 
acesso a recursos pode ser uma das explicações para as desigualdades encontradas na análise de desempenho em matemática.

\section{Discussão}

A Declaração da Educação para Todos (UNESCO, 1990) reafirma o princípio do direito fundamental de todos a uma educação básica de qualidade e que, para tanto, os serviços de educação devem ser expandidos e medidas consistentes devem ser tomadas para reduzir as disparidades. No Brasil, ações governamentais para expansão e acesso à educação são perceptíveis. Todavia, as ações para redução das disparidades ainda são incipientes.

Os resultados deste estudo apontam grandes desigualdades entre estudantes pobres e os estudantes de outros níveis socioeconômicos. Verificou-se que a chance de sucesso acadêmico de um estudante pobre é aproximadamente um terço da chance de um estudante de outro nível socioeconômico. Isso é um indicativo de que as disparidades sociais ainda se refletem em desigualdades educacionais e, consequentemente, parece apontar que a educação não tem cumprido um papel tão emancipador quanto se deseja.

O objetivo de apresentar os resultados em termos de razão de chance foi tornar mais transparente a magnitude das diferenças entre os alunos pobres e os alunos dos demais níveis socioeconômicos. Médias de desempenho menores entre os alunos pobres já eram sabidas, mas o nível de vulnerabilidade a que estão expostos esses alunos não era tão evidente. Por exemplo, é surpreendente saber que no Brasil há um sucesso relativo, entre alunos pobres e de outros grupos sociais, menor que $50 \%$. Um aspecto positivo é que essa não é uma condicionante de todas as UF/redes de ensino do país. Logo, há redes que têm promovido maior igualdade do que outras. No entanto, não há UF/rede que se destaque no 
Brasil por promover qualidade (alto percentual de alunos no nível adequado) com igualdade.

Adicionalmente, as análises de regressão no nível da escola corroboram os resultados encontrados no nível do aluno, apontando uma relação forte entre nível socioeconômico e desempenho matemático. Dessa forma, os alunos com maior nível socioeconômico tendem a estar na mesma escola e eles tendem a ter melhor desempenho. Willms (2006) denomina esse fenômeno de segregação horizontal. Um sistema segregado horizontalmente é aquele em que estudantes de diferentes contextos socioeconômicos estão segregados em escolas de modo a reproduzir as condições sociais e econômicas das localidades de sua residência. Além disso, os resultados de regressão também apontam que o sistema educacional brasileiro apresenta segregação vertical, uma vez que também se evidencia uma grande variabilidade no desempenho acadêmico entre escolas de mesmo nível socioeconômico.

Em termos de igualdade e equidade, os dois tipos de segregação são indesejáveis. Com base nos dados do PISA 2006, Willms (2010) identificou que o sistema escolar da América Latina tende a ser tanto verticalmente, quanto horizontalmente segregado. Ademais, o autor aponta que é comum que sistemas horizontalmente segregados sejam também verticalmente segregados, no caso dos dados do PISA 2006, a correlação desses dois tipos de segregação foi de 0,64 .

Quanto à equidade, verifica-se que os pilares fundamentais da educação não são garantidos a todos (Willms, 2015). Em termos de qualidade de ensino, os estudantes pobres têm menos acesso a escolas em que mais de $80 \%$ do currículo é cumprido e a escolas em que todos os alunos têm material didático. Além disso, os estudantes pobres têm também um tempo menor de aprendizado e menos acesso a recursos escolares. Resultados semelhantes foram encontrados no estudo de Willms, Tramonte, Duarte e Bos (2012). Esses pesquisadores analisaram os dados da Prova Brasil 2007, $5^{\circ}$ ano do ensino 
fundamental, e verificaram que estudantes pobres têm menos acesso a escolas com boa infraestrutura, seguras e com biblioteca bem equipada.

Esses resultados indicam problemas de igualdade de oportunidades no sistema educacional brasileiro. Para Rawls (2002), a igualdade de oportunidades vai além da distribuição igual de recursos, ela precisa ser compensatória, sendo mais vantajosa para os menos favorecidos. Bem diferente do que os resultados demonstraram. Como diz Soares (2007), considerando as grandes desigualdades sociais do nosso país, o objetivo do sistema educacional não pode ser apenas melhorar o nível de desempenho dos alunos de modo geral, mas também a equidade. Todavia, o sistema educacional brasileiro não tem conseguido prover nem melhoria no desempenho, nem equidade.

Os resultados mostram, além de problemas de equidade e igualdade, problemas gerais de acesso a recursos mínimos, como já apontado no Karino (2016). Esses resultados de certo modo nos fazem compreender o desempenho do Brasil em avaliações educacionais como o PISA. Considerando as diferenças de acesso a recursos, um raciocínio direto é imaginar que isso pode ser uma das causas das desigualdades no desempenho. Todavia, vale ressaltar que esses efeitos não são tão diretos, como já apontados em estudos anteriores (Strand, 2010). Além de recursos, o aprendizado depende de uma relação de interação. Dessa forma, sugere-se a realização de estudos que busquem mais bem investigar tais relações, sobretudo com a utilização da perspectiva do modelo de prosperidade educacional.

A comunidade científica possui corresponsabilidade sobre a ausência de políticas que buscam promover a igualdade e equidade do sistema educacional na medida em que não consegue prover subsídios necessários para auxiliar educadores e gestores. Esta pesquisa pretendeu contribuir com a construção de indicadores de igualdade e equidade do sistema educacional brasileiro. 
Por fim, espera-se ter contribuído no diagnóstico das desigualdades e inequidades existentes na educação brasileira e, ao demonstrar as diferenças por unidade da federação e rede administrativa, ter levantado a esperança de que são possíveis ações de melhoria. Investigar melhor os sistemas que têm conseguido maior igualdade e maior eficácia pode trazer indicações de ações de sucesso. Espera-se ainda ter chamado a atenção para o nível de vulnerabilidade a que estão expostos os estudantes pobres. É inadmissível em um sistema escolar um estudante aprender menos ou ter menos acesso a recursos por ser pobre.

Estudar igualdade e equidade é investir na geração de conhecimentos que possibilitem, no futuro, a oportunidade de aprendizado garantida a todos, sem distinção por etnia, nível socioeconômico ou qualquer outra característica. É investir no futuro de um país e na ideia de que a escola possa ser o mecanismo para desconstruir a estrutura de reprodução das relações entre as classes sociais. Mais do que resultados, a equidade no acesso à boa educação é uma questão de justiça (Levin, 2009).

\section{Referências}

Adams, J. (1965). Inequity in social exchange. Advances in Experimental Social Psychology, 2, 267-299.

Albanez, A., Ferreira, F., \& Franco, F. (2002). Qualidade e equidade no ensino fundamental brasileiro. Pesquisa e Planejamento Econômico, 32(3), 453-475.

Andrade, M., Franco, C., \& Carvalho, J. B. P. (2003). Gênero e desempenho em matemática ao final do ensino médio: quais as relações? Estudos em Avaliação Educacional, 27, 77-95.

Bali, V. A., \& Alvarez, R. M. (2004). The race gap in student achievement scores: Longitudinal evidence from a racially diverse school district. Policy Studies Journal, $32,393-415$.

Barros, R. P., Henriques, R., \& Mendonça, R. (2000). Desigualdade e pobreza no Brasil: Retrato de uma estabilidade inaceitável. Revista Brasileira de Ciências Sociais, 15(42), 123-142.

Cohen, E., \& Franco, R. (2013). Avaliação de projetos sociais. Petrópolis: Editora Vozes. 
Fernandes, R. (2007). Índice de Desenvolvimento da Educação Básica (IDEB): metas intermediárias para a sua trajetória no Brasil, estados, municípios e escolas. Brasília: INEP.

Fryer, R. G., \& Levitt, S. D. (2004). Understanding the black-white test score gap in the first two years of school. Review of Economics and Statistics, 86, 447-464.

INEP (2007). Nota metodológica sobre a compatibilização de desempenhos do PISA com a escala do SAEB. Brasília: Autor.

Karino, C. A. (2016). Pillars of good education: Analyzing the Brazilian school system. Manuscrito em preparação.

Koslinski, M. C., \& Alves, F. (2012). Novos olhares para as desigualdades de oportunidades educacionais: A segregação residencial e a relação favela-asfalto no contexto carioca. Educação \& Sociedade, 33(120), 805-831.

Leventhal, G. S., Popp, A. L., \& Sawyer, L. (1973). Equity or equality in children's allocation of rewards to other persons? Child Development, 44(4), 753-763.

Levin, H. M. (2009). The economic payoff to investing in educational justice. Educational Researcher, 38(1), 5-20.

Rawls, J. (2002). La justicia como equidad. Una reformulación. Barcelona: Paidós

Reynolds, D., Sammons, P., De Fraine, B., Van Damme, J., Townsend, T., Teddlie, C., \& Stringfield, S. (2014). Educational effectiveness research (EER): A state-of-the-art review. School Effectiveness and School Improvement: An International Journal of Research, Policy and Practice, 25(2), 197-230.

Santiago, A. R. F. (2012). Pedagogia crítica e educação emancipatória na escola pública: Um diálogo entre Paulo Freire e Boaventura Santos. In Trabalhos Completos, IX Seminário de pesquisa em educação da região sul. Caixas do Sul, RS: ANPED sul.

Soares Neto, J. J., Jesus, G. R., Karino, C. A., \& Andrade, D. F. (2013). Uma escala para medir a infraestrutura escolar. Estudos em Avaliação Educacional, 24(54), 78-99.

Soares, J. F. (2004a). Qualidade e equidade na educação brasileira: A evidência do SAEB2001. Archivos analíticos de políticas educativas, 12(38), 1-28.

Soares, J. F. (2004b). O efeito da escola no desempenho cognitivo de seus alunos. Revista Electronica Iberoamericana sobre Calidad, Eficacia y Cambio en Educación, 2(2), 83-104.

Soares, J. F. (2007). Melhoria do desempenho cognitivo dos alunos no ensino fundamental. Cadernos de Pesquisa, 37(130), 135-160.

Soares, J. F., \& Alves, M. T. G. (2003). Desigualdades raciais no sistema brasileiro de educação básica. Educação e Pesquisa, 29(1), 147-165. 
Soares, J. F., \& Andrade, R. J. de (2006). Nível socioeconômico, qualidade e equidade das escolas de Belo Horizonte. Ensaio: Avaliação de Políticas Públicas Educacionais, 14(50), 107-126.

Strand, S. (2010). Do some schools narrow the gap? Differential school effectiveness by ethnicity, gender, poverty, and prior achievement. School Effectiveness and School Improvement: An International Journal of Research, Policy and Practice, 21(3), 289314.

Todos pela Educação (2011). Educação: Uma agenda urgente. Brasília: Editora Moderna.

UNESCO (1990). Declaração mundial sobre educação para todos: Satisfação das necessidades básicas de aprendizagem. Jomtien: Autor.

Willms, J. D. (2006). Learning divides: Ten policy questions about the performance and equity of schools and schooling systems. Montreal, Quebec: UNESCO.

Willms, J. D. (2010). School composition and contextual effects on student outcomes. Teachers College Record, 112(4), 1008-1037.

Willms, J. D. (2015). Educational prosperity. Fredericton, NB: The Learning Bar Inc.

Willms, J. D., Durepos, D., López García, A. Y., Laurie, R., \& Tramonte, L. (2015). Design and composition of contextual questionnaires for the PISA for development study. Fredericton, NB: The Learning Bar Inc.

Willms, J. D., Tramonte, L., Duarte, J., \& Bos, S. (2012). Assessing educational equality and equity with large-scale assessment data: Brazil as a case study. Washington: Inter-American Development Bank. 
Anexo 1: Análise de igualdade e regressão

\begin{tabular}{|c|c|c|c|c|c|c|c|c|c|c|}
\hline \multirow[b]{2}{*}{ UF/Rede } & \multirow[b]{2}{*}{$\mathrm{N}$ alunos } & \multirow{2}{*}{$\begin{array}{c}\text { Total } \\
\% \\
\text { Sucesso }\end{array}$} & \multicolumn{2}{|c|}{ Grupo Pobre } & \multicolumn{2}{|c|}{ Demais Grupos } & \multicolumn{2}{|c|}{$\begin{array}{c}\text { Igualdade } \\
\text { (Pobres x Outros) } \\
\end{array}$} & \multicolumn{2}{|c|}{ Regressão } \\
\hline & & & $\begin{array}{l}\% \text { Abaixo } \\
\text { adequado }\end{array}$ & $\begin{array}{c}\% \\
\text { Sucesso }\end{array}$ & $\begin{array}{l}\% \text { Abaixo } \\
\text { adequado }\end{array}$ & $\begin{array}{c}\% \\
\text { Sucesso }\end{array}$ & $\begin{array}{l}\text { Sucesso } \\
\text { relativo }\end{array}$ & $\begin{array}{l}\text { Razão de } \\
\text { Chance de } \\
\text { sucesso }\end{array}$ & Slope & $\mathrm{R}^{2}$ \\
\hline Brasil & 1.908 .395 & $11 \%$ & $95 \%$ & $5 \%$ & $87 \%$ & $13 \%$ & 0,40 & 0,37 & 22,6 & 0,40 \\
\hline RO_Est & 12.752 & $9 \%$ & $94 \%$ & $6 \%$ & $91 \%$ & $9 \%$ & 0,63 & 0,61 & 15,9 & 0,17 \\
\hline RO_Mun & 4.629 & $6 \%$ & $95 \%$ & $5 \%$ & $93 \%$ & $7 \%$ & 0,72 & 0,70 & 7,4 & 0,03 \\
\hline AC_Est & 5.840 & $7 \%$ & $96 \%$ & $4 \%$ & $92 \%$ & $8 \%$ & 0,49 & 0,47 & 19,9 & 0,42 \\
\hline AC_Mun & 875 & $4 \%$ & $98 \%$ & $2 \%$ & $94 \%$ & $6 \%$ & 0,37 & 0,36 & 22,8 & 0,44 \\
\hline AM_Est & 29.239 & $9 \%$ & $93 \%$ & $7 \%$ & $91 \%$ & $9 \%$ & 0,74 & 0,72 & 15,2 & 0,11 \\
\hline AM_Mun & 10.100 & $4 \%$ & $97 \%$ & $3 \%$ & $95 \%$ & $5 \%$ & 0,62 & 0,61 & 12,0 & 0,17 \\
\hline RR_Est & 4.605 & $6 \%$ & $97 \%$ & $3 \%$ & $93 \%$ & $7 \%$ & 0,37 & 0,35 & 17,6 & 0,55 \\
\hline RR_Mun & 89 & $4 \%$ & $98 \%$ & $3 \%$ & $94 \%$ & $6 \%$ & 0,41 & 0,39 & 7,9 & 0,11 \\
\hline PA_Est & 26.677 & $4 \%$ & $98 \%$ & $2 \%$ & $95 \%$ & $5 \%$ & 0,45 & 0,44 & 15,3 & 0,32 \\
\hline PA_Mun & 39.683 & $5 \%$ & $97 \%$ & $3 \%$ & $94 \%$ & $6 \%$ & 0,50 & 0,49 & 13,9 & 0,22 \\
\hline AP_Est & 6.718 & $3 \%$ & $98 \%$ & $2 \%$ & $96 \%$ & $4 \%$ & 0,45 & 0,44 & 15,1 & 0,29 \\
\hline AP_Mun & 628 & $3 \%$ & $99 \%$ & $1 \%$ & $96 \%$ & $4 \%$ & 0,30 & 0,29 & $-0,7$ & 0,00 \\
\hline TOC_Est & 14.015 & $7 \%$ & $95 \%$ & $5 \%$ & $91 \%$ & $9 \%$ & 0,49 & 0,47 & 24,6 & 0,34 \\
\hline TOC_Mun & 2.919 & $10 \%$ & $95 \%$ & $5 \%$ & $86 \%$ & $14 \%$ & 0,37 & 0,33 & 32,3 & 0,61 \\
\hline MA_Est & 27.286 & $5 \%$ & $97 \%$ & $3 \%$ & $93 \%$ & $7 \%$ & 0,42 & 0,40 & 21,7 & 0,32 \\
\hline MA_Mun & 39.225 & $3 \%$ & $98 \%$ & $2 \%$ & $94 \%$ & $6 \%$ & 0,38 & 0,36 & 18,3 & 0,24 \\
\hline PI_Est & 8.512 & $7 \%$ & $95 \%$ & $5 \%$ & $92 \%$ & $8 \%$ & 0,55 & 0,53 & $-1,1$ & 0,00 \\
\hline PI_Mun & 16.587 & $7 \%$ & $95 \%$ & $5 \%$ & $89 \%$ & $11 \%$ & 0,44 & 0,41 & 22,8 & 0,30 \\
\hline CE_Est & 28.687 & $7 \%$ & $95 \%$ & $5 \%$ & $91 \%$ & $9 \%$ & 0,60 & 0,57 & 21,8 & 0,17 \\
\hline CE_Mun & 68.273 & $6 \%$ & $96 \%$ & $4 \%$ & $92 \%$ & $8 \%$ & 0,53 & 0,51 & 20,6 & 0,16 \\
\hline RN_Est & 11.928 & $7 \%$ & $96 \%$ & $4 \%$ & $92 \%$ & $8 \%$ & 0,50 & 0,48 & 22,3 & 0,20 \\
\hline RN_Mun & 12.908 & $6 \%$ & $97 \%$ & $3 \%$ & $92 \%$ & $8 \%$ & 0,38 & 0,36 & 21,0 & 0,15 \\
\hline PB_Est & 16.833 & $5 \%$ & $97 \%$ & $3 \%$ & $93 \%$ & $7 \%$ & 0,39 & 0,38 & 21,3 & 0,23 \\
\hline PB_Mun & 16.344 & $4 \%$ & $97 \%$ & $3 \%$ & $94 \%$ & $6 \%$ & 0,48 & 0,46 & 19,2 & 0,20 \\
\hline PE_Est & 46.408 & $6 \%$ & $96 \%$ & $4 \%$ & $93 \%$ & $7 \%$ & 0,49 & 0,48 & 18,3 & 0,14 \\
\hline PE_Mun & 33.275 & $4 \%$ & $98 \%$ & $2 \%$ & $95 \%$ & $5 \%$ & 0,48 & 0,46 & 14,1 & 0,29 \\
\hline AL_Est & 11.427 & $4 \%$ & $98 \%$ & $2 \%$ & $95 \%$ & $5 \%$ & 0,48 & 0,47 & 23,8 & 0,23 \\
\hline AL_Mun & 19.840 & $3 \%$ & $97 \%$ & $3 \%$ & $95 \%$ & $5 \%$ & 0,51 & 0,49 & 19,1 & 0,19 \\
\hline SE_Est & 8.686 & $5 \%$ & $96 \%$ & $4 \%$ & $94 \%$ & $6 \%$ & 0,60 & 0,59 & 15,2 & 0,11 \\
\hline SE_Mun & 6.776 & $5 \%$ & $96 \%$ & $4 \%$ & $93 \%$ & $7 \%$ & 0,58 & 0,57 & 14,2 & 0,09 \\
\hline BA_Est & 52.670 & $5 \%$ & $97 \%$ & $3 \%$ & $94 \%$ & $6 \%$ & 0,47 & 0,46 & 22,1 & 0,24 \\
\hline BA_Mun & 75.904 & $4 \%$ & $97 \%$ & $3 \%$ & $94 \%$ & $6 \%$ & 0,44 & 0,42 & 14,8 & 0,15 \\
\hline MG_Est & 159.862 & $19 \%$ & $88 \%$ & $12 \%$ & $78 \%$ & $22 \%$ & 0,56 & 0,50 & 18,3 & 0,22 \\
\hline MG_Mun & 59.156 & $17 \%$ & $90 \%$ & $10 \%$ & $81 \%$ & $19 \%$ & 0,52 & 0,47 & 21,6 & 0,25 \\
\hline ES_Est & 11.563 & $12 \%$ & $92 \%$ & $8 \%$ & $87 \%$ & $13 \%$ & 0,58 & 0,54 & 11,7 & 0,04 \\
\hline ES_Mun & 19.498 & $14 \%$ & $91 \%$ & $9 \%$ & $85 \%$ & $15 \%$ & 0,58 & 0,54 & 20,6 & 0,15 \\
\hline RJ_Est & 49.522 & $9 \%$ & $94 \%$ & $6 \%$ & $91 \%$ & $9 \%$ & 0,67 & 0,65 & 21,1 & 0,08 \\
\hline RJ_Mun & 68.750 & $12 \%$ & $92 \%$ & $8 \%$ & $87 \%$ & $13 \%$ & 0,59 & 0,56 & 28,7 & 0,21 \\
\hline SP_Est & 357.581 & $12 \%$ & $93 \%$ & $7 \%$ & $88 \%$ & $12 \%$ & 0,54 & 0,51 & 23,6 & 0,20 \\
\hline SP_Mun & 103.163 & $14 \%$ & $92 \%$ & $8 \%$ & $85 \%$ & $15 \%$ & 0,54 & 0,50 & 30,0 & 0,26 \\
\hline PR_Est & 119.281 & $13 \%$ & $93 \%$ & $7 \%$ & $86 \%$ & $14 \%$ & 0,45 & 0,41 & 21,6 & 0,34 \\
\hline PR_Mun & 3.458 & $15 \%$ & $92 \%$ & $8 \%$ & $84 \%$ & $16 \%$ & 0,51 & 0,46 & 23,6 & 0,31 \\
\hline SC_Est & 42.340 & $14 \%$ & $93 \%$ & $7 \%$ & $86 \%$ & $14 \%$ & 0,51 & 0,47 & 21,3 & 0,18 \\
\hline SC_Mun & 24.942 & $18 \%$ & $92 \%$ & $8 \%$ & $81 \%$ & $19 \%$ & 0,41 & 0,36 & 21,3 & 0,18 \\
\hline RS_Est & 55.244 & $18 \%$ & $90 \%$ & $10 \%$ & $82 \%$ & $18 \%$ & 0,53 & 0,48 & 22,5 & 0,19 \\
\hline RS_Mun & 36.198 & $14 \%$ & $93 \%$ & $7 \%$ & $85 \%$ & $15 \%$ & 0,48 & 0,44 & 27,5 & 0,27 \\
\hline MS_Est & 14.784 & $12 \%$ & $93 \%$ & $7 \%$ & $87 \%$ & $13 \%$ & 0,49 & 0,45 & 25,3 & 0,37 \\
\hline MS_Mun & 8.652 & $15 \%$ & $92 \%$ & $8 \%$ & $83 \%$ & $17 \%$ & 0,50 & 0,45 & 28,6 & 0,39 \\
\hline MT_Est & 23.082 & $9 \%$ & $93 \%$ & $7 \%$ & $90 \%$ & $10 \%$ & 0,66 & 0,64 & 16,8 & 0,16 \\
\hline MT_Mun & 8.689 & $10 \%$ & $95 \%$ & $5 \%$ & $88 \%$ & $12 \%$ & 0,45 & 0,42 & 16,1 & 0,17 \\
\hline GO_Est & 46.185 & $7 \%$ & $95 \%$ & $5 \%$ & $92 \%$ & $8 \%$ & 0,54 & 0,52 & 17,4 & 0,18 \\
\hline GO_Mun & 14.685 & $9 \%$ & $94 \%$ & $6 \%$ & $90 \%$ & $10 \%$ & 0,56 & 0,53 & 25,5 & 0,25 \\
\hline DF_Est & 18.398 & $13 \%$ & $91 \%$ & $9 \%$ & $86 \%$ & $14 \%$ & 0,61 & 0,58 & 16,1 & 0,18 \\
\hline
\end{tabular}


Anexo 2: Análise de equidade - pilar qualidade do ensino (Parte 1)

\begin{tabular}{|c|c|c|c|c|c|c|c|c|c|c|c|c|}
\hline \multirow[b]{2}{*}{ UF/Rede } & \multicolumn{4}{|c|}{ Professor Corrige Dever } & \multicolumn{4}{|c|}{ Currículo Adequado } & \multicolumn{4}{|c|}{$\%$ Currículo } \\
\hline & $\begin{array}{l}\text { Grupo } \\
\text { pobre }\end{array}$ & $\begin{array}{c}\text { Demais } \\
\text { grupos }\end{array}$ & $\begin{array}{l}\text { Acesso } \\
\text { relativo }\end{array}$ & $\begin{array}{l}\text { Razão de } \\
\text { chance } \\
\text { (Acesso) }\end{array}$ & $\begin{array}{l}\text { Grupo } \\
\text { pobre }\end{array}$ & $\begin{array}{c}\text { Demais } \\
\text { grupos }\end{array}$ & $\begin{array}{l}\text { Acesso } \\
\text { relativo }\end{array}$ & $\begin{array}{l}\text { Razão de } \\
\text { chance } \\
\text { (Acesso) }\end{array}$ & $\begin{array}{l}\text { Grupo } \\
\text { pobre }\end{array}$ & $\begin{array}{c}\text { Demais } \\
\text { grupos }\end{array}$ & $\begin{array}{l}\text { Acesso } \\
\text { relativo }\end{array}$ & $\begin{array}{l}\text { Razão de } \\
\text { chance } \\
\text { (Acesso) }\end{array}$ \\
\hline Brasil & $33 \%$ & $29 \%$ & 1,12 & 1,17 & $62 \%$ & $63 \%$ & 0,99 & 0,96 & $10 \%$ & $14 \%$ & 0,71 & 0,68 \\
\hline RO_Est & $28 \%$ & $26 \%$ & 1,06 & 1,08 & $59 \%$ & $61 \%$ & 0,97 & 0,92 & $6 \%$ & $11 \%$ & 0,60 & 0,57 \\
\hline RO_Mun & $29 \%$ & $23 \%$ & 1,27 & 1,37 & $66 \%$ & $60 \%$ & 1,10 & 1,30 & $10 \%$ & $11 \%$ & 0,96 & 0,95 \\
\hline AC_Est & $15 \%$ & $13 \%$ & 1,14 & 1,16 & $51 \%$ & $49 \%$ & 1,05 & 1,10 & $16 \%$ & $20 \%$ & 0,81 & 0,77 \\
\hline AC_Mun & $22 \%$ & $22 \%$ & 0,97 & 0,96 & $78 \%$ & $78 \%$ & 0,99 & 0,97 & $24 \%$ & $34 \%$ & 0,71 & 0,62 \\
\hline AM_Est & $10 \%$ & $8 \%$ & 1,21 & 1,24 & $64 \%$ & $63 \%$ & 1,02 & 1,07 & $5 \%$ & $9 \%$ & 0,58 & 0,55 \\
\hline AM_Mun & $10 \%$ & $9 \%$ & 1,05 & 1,05 & $56 \%$ & $56 \%$ & 1,00 & 1,00 & $4 \%$ & $2 \%$ & 1,71 & 1,74 \\
\hline RR_Est & $5 \%$ & $7 \%$ & 0,73 & 0,71 & $62 \%$ & $64 \%$ & 0,98 & 0,94 & $8 \%$ & $11 \%$ & 0,78 & 0,76 \\
\hline RR_Mun & $33 \%$ & $18 \%$ & 1,77 & 2,14 & $33 \%$ & $18 \%$ & 1,77 & 2,14 & $0 \%$ & $0 \%$ & & \\
\hline PA_Est & $11 \%$ & $11 \%$ & 0,95 & 0,95 & $50 \%$ & $51 \%$ & 0,98 & 0,96 & $8 \%$ & $9 \%$ & 0,86 & 0,85 \\
\hline PA_Mun & $20 \%$ & $21 \%$ & 0,93 & 0,92 & $55 \%$ & $54 \%$ & 1,02 & 1,05 & $14 \%$ & $17 \%$ & 0,83 & 0,80 \\
\hline AP_Est & $5 \%$ & $2 \%$ & 2,27 & 2,33 & $56 \%$ & $55 \%$ & 1,02 & 1,04 & $9 \%$ & $8 \%$ & 1,14 & 1,16 \\
\hline AP_Mun & $12 \%$ & $4 \%$ & 2,75 & 2,99 & $60 \%$ & $68 \%$ & 0,88 & 0,71 & $6 \%$ & $10 \%$ & 0,58 & 0,55 \\
\hline TO_Est & $25 \%$ & $28 \%$ & 0,90 & 0,87 & $80 \%$ & $80 \%$ & 1,00 & 0,98 & $14 \%$ & $19 \%$ & 0,74 & 0,70 \\
\hline TO_Mun & $39 \%$ & $31 \%$ & 1,25 & 1,41 & $80 \%$ & $77 \%$ & 1,03 & 1,17 & $11 \%$ & $24 \%$ & 0,48 & 0,41 \\
\hline MA_Est & $24 \%$ & $25 \%$ & 1,00 & 1,00 & $68 \%$ & $68 \%$ & 1,00 & 1,00 & $8 \%$ & $11 \%$ & 0,75 & 0,72 \\
\hline MA_Mun & $18 \%$ & $19 \%$ & 0,94 & 0,93 & $62 \%$ & $64 \%$ & 0,96 & 0,89 & $7 \%$ & $12 \%$ & 0,62 & 0,59 \\
\hline PI_Est & $42 \%$ & $38 \%$ & 1,11 & 1,19 & $65 \%$ & $65 \%$ & 1,01 & 1,02 & $20 \%$ & $21 \%$ & 0,96 & 0,95 \\
\hline PI_Mun & $41 \%$ & $39 \%$ & 1,05 & 1,08 & $69 \%$ & $69 \%$ & 0,99 & 0,98 & $22 \%$ & $25 \%$ & 0,87 & 0,83 \\
\hline CE_Est & $33 \%$ & $29 \%$ & 1,13 & 1,20 & $63 \%$ & $62 \%$ & 1,02 & 1,05 & $5 \%$ & $5 \%$ & 1,08 & 1,09 \\
\hline CE_Mun & $36 \%$ & $34 \%$ & 1,05 & 1,08 & $61 \%$ & $59 \%$ & 1,04 & 1,09 & $7 \%$ & $9 \%$ & 0,82 & 0,81 \\
\hline RN_Est & $23 \%$ & $25 \%$ & 0,94 & 0,92 & $54 \%$ & $54 \%$ & 1,00 & 1,01 & $5 \%$ & $7 \%$ & 0,73 & 0,71 \\
\hline RN_Mun & $24 \%$ & $23 \%$ & 1,05 & 1,07 & $53 \%$ & $57 \%$ & 0,93 & 0,86 & $5 \%$ & $8 \%$ & 0,67 & 0,65 \\
\hline PB_Est & $25 \%$ & $22 \%$ & 1,16 & 1,21 & $52 \%$ & $57 \%$ & 0,91 & 0,82 & $8 \%$ & $9 \%$ & 0,84 & 0,83 \\
\hline PB_Mun & $36 \%$ & $32 \%$ & 1,11 & 1,17 & $61 \%$ & $63 \%$ & 0,98 & 0,94 & $11 \%$ & $15 \%$ & 0,75 & 0,72 \\
\hline PE_Est & $20 \%$ & $18 \%$ & 1,11 & 1,14 & $58 \%$ & $57 \%$ & 1,03 & 1,06 & $8 \%$ & $9 \%$ & 0,89 & 0,88 \\
\hline PE_Mun & $25 \%$ & $21 \%$ & 1,22 & 1,29 & $55 \%$ & $51 \%$ & 1,08 & 1,17 & $7 \%$ & $8 \%$ & 0,93 & 0,93 \\
\hline AL_Est & $20 \%$ & $19 \%$ & 1,05 & 1,06 & $76 \%$ & $77 \%$ & 0,99 & 0,94 & $6 \%$ & $5 \%$ & 1,38 & 1,41 \\
\hline AL_Mun & $26 \%$ & $24 \%$ & 1,11 & 1,15 & $63 \%$ & $58 \%$ & 1,08 & 1,21 & $4 \%$ & $5 \%$ & 0,84 & 0,84 \\
\hline SE_Est & $44 \%$ & $41 \%$ & 1,07 & 1,13 & $61 \%$ & $57 \%$ & 1,06 & 1,16 & $13 \%$ & $12 \%$ & 1,09 & 1,11 \\
\hline SE_Mun & $52 \%$ & $48 \%$ & 1,08 & 1,17 & $52 \%$ & $59 \%$ & 0,88 & 0,75 & $11 \%$ & $14 \%$ & 0,74 & 0,71 \\
\hline BA_Est & $46 \%$ & $42 \%$ & 1,08 & 1,14 & $54 \%$ & $53 \%$ & 1,02 & 1,05 & $7 \%$ & $9 \%$ & 0,74 & 0,72 \\
\hline BA_Mun & $58 \%$ & $52 \%$ & 1,12 & 1,28 & $55 \%$ & $55 \%$ & 1,01 & 1,02 & $7 \%$ & $8 \%$ & 0,85 & 0,84 \\
\hline MG_Est & $45 \%$ & $41 \%$ & 1,10 & 1,17 & $77 \%$ & $75 \%$ & 1,02 & 1,10 & $13 \%$ & $17 \%$ & 0,73 & 0,69 \\
\hline MG_Mun & $48 \%$ & $40 \%$ & 1,21 & 1,39 & $65 \%$ & $61 \%$ & 1,07 & 1,20 & $15 \%$ & $17 \%$ & 0,88 & 0,86 \\
\hline ES_Est & $28 \%$ & $27 \%$ & 1,05 & 1,07 & $70 \%$ & $74 \%$ & 0,96 & 0,86 & $18 \%$ & $18 \%$ & 1,01 & 1,01 \\
\hline ES_Mun & $54 \%$ & $50 \%$ & 1,09 & 1,20 & $65 \%$ & $62 \%$ & 1,05 & 1,13 & $13 \%$ & $16 \%$ & 0,78 & 0,75 \\
\hline RJ_Est & $35 \%$ & $35 \%$ & 1,01 & 1,01 & $60 \%$ & $59 \%$ & 1,03 & 1,07 & $13 \%$ & $13 \%$ & 0,98 & 0,98 \\
\hline RJ_Mun & $38 \%$ & $37 \%$ & 1,03 & 1,05 & $55 \%$ & $55 \%$ & 1,01 & 1,02 & $14 \%$ & $15 \%$ & 0,98 & 0,98 \\
\hline SP_Est & $13 \%$ & $15 \%$ & 0,88 & 0,86 & $59 \%$ & $61 \%$ & 0,98 & 0,94 & $8 \%$ & $10 \%$ & 0,82 & 0,80 \\
\hline SP_Mun & $25 \%$ & $25 \%$ & 0,98 & 0,97 & $72 \%$ & $72 \%$ & 1,01 & 1,04 & $21 \%$ & $23 \%$ & 0,93 & 0,91 \\
\hline PR_Est & $26 \%$ & $25 \%$ & 1,04 & 1,06 & $74 \%$ & $73 \%$ & 1,02 & 1,07 & $9 \%$ & $12 \%$ & 0,75 & 0,73 \\
\hline PR_Mun & $39 \%$ & $23 \%$ & 1,70 & 2,16 & $67 \%$ & $65 \%$ & 1,04 & 1,12 & $8 \%$ & $11 \%$ & 0,72 & 0,70 \\
\hline SC_Est & $45 \%$ & $42 \%$ & 1,05 & 1,09 & $61 \%$ & $58 \%$ & 1,06 & 1,16 & $13 \%$ & $15 \%$ & 0,86 & 0,84 \\
\hline SC_Mun & $48 \%$ & $50 \%$ & 0,96 & 0,91 & $61 \%$ & $61 \%$ & 0,99 & 0,98 & $19 \%$ & $26 \%$ & 0,74 & 0,68 \\
\hline RS_Est & $50 \%$ & $48 \%$ & 1,04 & 1,08 & $62 \%$ & $62 \%$ & 1,00 & 1,01 & $25 \%$ & $29 \%$ & 0,87 & 0,82 \\
\hline RS_Mun & $53 \%$ & $53 \%$ & 1,00 & 1,01 & $62 \%$ & $65 \%$ & 0,96 & 0,90 & $24 \%$ & $26 \%$ & 0,93 & 0,91 \\
\hline MS_Est & $48 \%$ & $48 \%$ & 1,01 & 1,01 & $62 \%$ & $61 \%$ & 1,01 & 1,03 & $7 \%$ & $9 \%$ & 0,82 & 0,80 \\
\hline MS_Mun & $48 \%$ & $46 \%$ & 1,04 & 1,07 & $65 \%$ & $66 \%$ & 0,99 & 0,97 & $9 \%$ & $14 \%$ & 0,64 & 0,61 \\
\hline MT_Est & $23 \%$ & $22 \%$ & 1,07 & 1,09 & $64 \%$ & $61 \%$ & 1,05 & 1,13 & $14 \%$ & $13 \%$ & 1,09 & 1,11 \\
\hline MT_Mun & $33 \%$ & $31 \%$ & 1,04 & 1,06 & $67 \%$ & $67 \%$ & 1,01 & 1,02 & $13 \%$ & $21 \%$ & 0,59 & 0,53 \\
\hline GO_Est & $45 \%$ & $43 \%$ & 1,05 & 1,09 & $61 \%$ & $60 \%$ & 1,01 & 1,02 & $9 \%$ & $13 \%$ & 0,67 & 0,64 \\
\hline GO_Mun & $48 \%$ & $49 \%$ & 0,97 & 0,95 & $62 \%$ & $60 \%$ & 1,04 & 1,12 & $13 \%$ & $15 \%$ & 0,88 & 0,87 \\
\hline DF_Est & $16 \%$ & $16 \%$ & 0,99 & 0,99 & $56 \%$ & $56 \%$ & 1,00 & 1,00 & $17 \%$ & $18 \%$ & 0,93 & 0,91 \\
\hline
\end{tabular}


Anexo 2: Análise de equidade - pilar qualidade do ensino (Parte 2)

\begin{tabular}{|c|c|c|c|c|c|c|c|c|c|c|c|c|}
\hline \multirow[b]{2}{*}{ UF/Rede } & \multicolumn{4}{|c|}{ Escolaridade Professor } & \multicolumn{4}{|c|}{ Escolaridade Diretor } & \multicolumn{4}{|c|}{ Material Didático } \\
\hline & $\begin{array}{l}\text { Grupo } \\
\text { pobre }\end{array}$ & $\begin{array}{l}\text { Demais } \\
\text { grupos }\end{array}$ & $\begin{array}{l}\text { Acesso } \\
\text { relativo }\end{array}$ & $\begin{array}{l}\text { Razão de } \\
\text { chance } \\
\text { (Acesso) }\end{array}$ & $\begin{array}{l}\text { Grupo } \\
\text { pobre }\end{array}$ & $\begin{array}{c}\text { Demais } \\
\text { grupos }\end{array}$ & $\begin{array}{l}\text { Acesso } \\
\text { relativo }\end{array}$ & $\begin{array}{l}\text { Razão de } \\
\text { chance } \\
\text { (Acesso) }\end{array}$ & $\begin{array}{l}\text { Grupo } \\
\text { pobre }\end{array}$ & $\begin{array}{l}\text { Demais } \\
\text { grupos }\end{array}$ & $\begin{array}{l}\text { Acesso } \\
\text { relativo }\end{array}$ & $\begin{array}{l}\text { Razão de } \\
\text { chance } \\
\text { (Acesso) }\end{array}$ \\
\hline Brasil & $27 \%$ & $30 \%$ & 0,90 & 0,86 & $66 \%$ & $74 \%$ & 0,89 & 0,69 & $43 \%$ & $49 \%$ & 0,88 & 0,79 \\
\hline RO_Est & $42 \%$ & $41 \%$ & 1,02 & 1,03 & $91 \%$ & $89 \%$ & 1,02 & 1,19 & $38 \%$ & $44 \%$ & 0,86 & 0,77 \\
\hline RO_Mun & $59 \%$ & $56 \%$ & 1,05 & 1,12 & $77 \%$ & $78 \%$ & 0,98 & 0,92 & $42 \%$ & $46 \%$ & 0,92 & 0,87 \\
\hline AC_Est & $21 \%$ & $22 \%$ & 0,96 & 0,95 & $94 \%$ & $97 \%$ & 0,97 & 0,53 & $51 \%$ & $53 \%$ & 0,96 & 0,92 \\
\hline AC_Mun & $39 \%$ & $48 \%$ & 0,81 & 0,69 & $52 \%$ & $51 \%$ & 1,03 & 1,06 & $37 \%$ & $34 \%$ & 1,07 & 1,11 \\
\hline AM_Est & $16 \%$ & $20 \%$ & 0,80 & 0,76 & $79 \%$ & $88 \%$ & 0,91 & 0,55 & $25 \%$ & $26 \%$ & 0,96 & 0,95 \\
\hline AM_Mun & $19 \%$ & $16 \%$ & 1,17 & 1,21 & $63 \%$ & $72 \%$ & 0,88 & 0,67 & $16 \%$ & $20 \%$ & 0,78 & 0,74 \\
\hline RR_Est & $44 \%$ & $48 \%$ & 0,90 & 0,83 & $81 \%$ & $87 \%$ & 0,93 & 0,62 & $35 \%$ & $46 \%$ & 0,75 & 0,62 \\
\hline RR_Mun & $100 \%$ & $100 \%$ & 1,00 & 1,00 & $0 \%$ & $0 \%$ & & & $0 \%$ & $0 \%$ & & \\
\hline PA_Est & $32 \%$ & $33 \%$ & 0,97 & 0,95 & $73 \%$ & $83 \%$ & 0,88 & 0,56 & $29 \%$ & $35 \%$ & 0,85 & 0,79 \\
\hline PA_Mun & $18 \%$ & $22 \%$ & 0,82 & 0,78 & $49 \%$ & $56 \%$ & 0,88 & 0,76 & $25 \%$ & $30 \%$ & 0,83 & 0,78 \\
\hline AP_Est & $12 \%$ & $19 \%$ & 0,64 & 0,59 & $59 \%$ & $73 \%$ & 0,81 & 0,54 & $32 \%$ & $31 \%$ & 1,04 & 1,06 \\
\hline AP_Mun & $6 \%$ & $2 \%$ & 3,07 & 3,20 & $15 \%$ & $26 \%$ & 0,58 & 0,50 & $26 \%$ & $33 \%$ & 0,77 & 0,69 \\
\hline TO_Est & $31 \%$ & $32 \%$ & 0,98 & 0,97 & $84 \%$ & $86 \%$ & 0,98 & 0,87 & $59 \%$ & $62 \%$ & 0,95 & 0,88 \\
\hline TO_Mun & $26 \%$ & $35 \%$ & 0,75 & 0,66 & $55 \%$ & $77 \%$ & 0,72 & 0,38 & $48 \%$ & $53 \%$ & 0,90 & 0,81 \\
\hline MA_Est & $28 \%$ & $31 \%$ & 0,90 & 0,86 & $61 \%$ & $64 \%$ & 0,95 & 0,87 & $47 \%$ & $57 \%$ & 0,82 & 0,66 \\
\hline MA_Mun & $27 \%$ & $31 \%$ & 0,88 & 0,84 & $45 \%$ & $52 \%$ & 0,85 & 0,73 & $29 \%$ & $31 \%$ & 0,95 & 0,93 \\
\hline PI_Est & $24 \%$ & $26 \%$ & 0,91 & 0,88 & $69 \%$ & $67 \%$ & 1,02 & 1,07 & $68 \%$ & $72 \%$ & 0,95 & 0,85 \\
\hline PI_Mun & $34 \%$ & $39 \%$ & 0,88 & 0,81 & $51 \%$ & $67 \%$ & 0,77 & 0,52 & $63 \%$ & $62 \%$ & 1,01 & 1,03 \\
\hline CE_Est & $22 \%$ & $23 \%$ & 0,95 & 0,93 & $96 \%$ & $97 \%$ & 0,99 & 0,75 & $64 \%$ & $63 \%$ & 1,01 & 1,04 \\
\hline CE_Mun & $26 \%$ & $28 \%$ & 0,90 & 0,87 & $65 \%$ & $72 \%$ & 0,90 & 0,72 & $50 \%$ & $53 \%$ & 0,95 & 0,89 \\
\hline RN_Est & $14 \%$ & $12 \%$ & 1,12 & 1,14 & $60 \%$ & $58 \%$ & 1,04 & 1,09 & $48 \%$ & $56 \%$ & 0,86 & 0,72 \\
\hline RN_Mun & $21 \%$ & $24 \%$ & 0,87 & 0,83 & $58 \%$ & $68 \%$ & 0,85 & 0,64 & $27 \%$ & $29 \%$ & 0,93 & 0,91 \\
\hline PB_Est & $10 \%$ & $12 \%$ & 0,83 & 0,82 & $47 \%$ & $53 \%$ & 0,89 & 0,79 & $42 \%$ & $42 \%$ & 0,98 & 0,97 \\
\hline PB_Mun & $23 \%$ & $28 \%$ & 0,82 & 0,77 & $57 \%$ & $62 \%$ & 0,92 & 0,82 & $41 \%$ & $47 \%$ & 0,88 & 0,79 \\
\hline PE_Est & $31 \%$ & $32 \%$ & 0,98 & 0,97 & $82 \%$ & $80 \%$ & 1,01 & 1,08 & $37 \%$ & $37 \%$ & 1,00 & 1,00 \\
\hline PE_Mun & $37 \%$ & $37 \%$ & 1,00 & 1,01 & $73 \%$ & $75 \%$ & 0,98 & 0,92 & $30 \%$ & $31 \%$ & 0,95 & 0,93 \\
\hline AL_Est & $34 \%$ & $38 \%$ & 0,91 & 0,87 & $71 \%$ & $82 \%$ & 0,86 & 0,53 & $15 \%$ & $20 \%$ & 0,75 & 0,71 \\
\hline AL_Mun & $31 \%$ & $32 \%$ & 0,99 & 0,99 & $46 \%$ & $49 \%$ & 0,94 & 0,88 & $12 \%$ & $11 \%$ & 1,15 & 1,17 \\
\hline SE_Est & $24 \%$ & $28 \%$ & 0,85 & 0,80 & $78 \%$ & $76 \%$ & 1,02 & 1,11 & $24 \%$ & $31 \%$ & 0,79 & 0,72 \\
\hline SE_Mun & $30 \%$ & $35 \%$ & 0,86 & 0,79 & $56 \%$ & $65 \%$ & 0,86 & 0,68 & $16 \%$ & $12 \%$ & 1,32 & 1,38 \\
\hline BA_Est & $30 \%$ & $34 \%$ & 0,88 & 0,83 & $70 \%$ & $77 \%$ & 0,90 & 0,68 & $36 \%$ & $35 \%$ & 1,03 & 1,04 \\
\hline BA_Mun & $13 \%$ & $18 \%$ & 0,72 & 0,68 & $35 \%$ & $48 \%$ & 0,73 & 0,58 & $33 \%$ & $36 \%$ & 0,92 & 0,88 \\
\hline MG_Est & $29 \%$ & $34 \%$ & 0,86 & 0,80 & $77 \%$ & $81 \%$ & 0,96 & 0,81 & $63 \%$ & $65 \%$ & 0,96 & 0,90 \\
\hline MG_Mun & $30 \%$ & $37 \%$ & 0,81 & 0,73 & $63 \%$ & $74 \%$ & 0,85 & 0,60 & $48 \%$ & $57 \%$ & 0,85 & 0,71 \\
\hline ES_Est & $64 \%$ & $69 \%$ & 0,93 & 0,81 & $98 \%$ & $98 \%$ & 0,99 & 0,78 & $51 \%$ & $53 \%$ & 0,96 & 0,92 \\
\hline ES_Mun & $69 \%$ & $74 \%$ & 0,94 & 0,80 & $92 \%$ & $94 \%$ & 0,97 & 0,68 & $48 \%$ & $52 \%$ & 0,93 & 0,86 \\
\hline RJ_Est & $25 \%$ & $24 \%$ & 1,01 & 1,01 & $67 \%$ & $68 \%$ & 0,98 & 0,94 & $39 \%$ & $40 \%$ & 0,97 & 0,95 \\
\hline RJ_Mun & $30 \%$ & $29 \%$ & 1,05 & 1,07 & $54 \%$ & $54 \%$ & 1,00 & 1,01 & $33 \%$ & $35 \%$ & 0,93 & 0,89 \\
\hline SP_Est & $10 \%$ & $10 \%$ & 0,95 & 0,94 & $68 \%$ & $69 \%$ & 0,99 & 0,95 & $53 \%$ & $54 \%$ & 0,98 & 0,96 \\
\hline SP_Mun & $23 \%$ & $26 \%$ & 0,91 & 0,88 & $66 \%$ & $65 \%$ & 1,00 & 1,01 & $50 \%$ & $50 \%$ & 1,00 & 1,01 \\
\hline PR_Est & $59 \%$ & $56 \%$ & 1,05 & 1,11 & $97 \%$ & $96 \%$ & 1,00 & 1,15 & $57 \%$ & $58 \%$ & 0,99 & 0,98 \\
\hline PR_Mun & $48 \%$ & $64 \%$ & 0,75 & 0,52 & $84 \%$ & $87 \%$ & 0,96 & 0,73 & $50 \%$ & $43 \%$ & 1,17 & 1,34 \\
\hline SC_Est & $69 \%$ & $64 \%$ & 1,07 & 1,22 & $100 \%$ & $100 \%$ & 1,00 & 1,00 & $48 \%$ & $49 \%$ & 0,98 & 0,95 \\
\hline SC_Mun & $60 \%$ & $58 \%$ & 1,03 & 1,08 & & & & & $45 \%$ & $47 \%$ & 0,96 & 0,93 \\
\hline RS_Est & $35 \%$ & $32 \%$ & 1,09 & 1,14 & $74 \%$ & $73 \%$ & 1,02 & 1,08 & $51 \%$ & $56 \%$ & 0,92 & 0,84 \\
\hline RS_Mun & $43 \%$ & $47 \%$ & 0,92 & 0,87 & $73 \%$ & $74 \%$ & 0,98 & 0,94 & $42 \%$ & $45 \%$ & 0,94 & 0,89 \\
\hline MS_Est & $30 \%$ & $31 \%$ & 0,98 & 0,97 & $74 \%$ & $75 \%$ & 0,98 & 0,91 & $43 \%$ & $46 \%$ & 0,93 & 0,88 \\
\hline MS_Mun & $35 \%$ & $32 \%$ & 1,11 & 1,18 & $88 \%$ & $90 \%$ & 0,98 & 0,83 & $44 \%$ & $46 \%$ & 0,95 & 0,92 \\
\hline MT_Est & $35 \%$ & $34 \%$ & 1,03 & 1,05 & $83 \%$ & $85 \%$ & 0,98 & 0,89 & $30 \%$ & $32 \%$ & 0,93 & 0,90 \\
\hline MT_Mun & $44 \%$ & $54 \%$ & 0,81 & 0,66 & $79 \%$ & $89 \%$ & 0,89 & 0,46 & $28 \%$ & $33 \%$ & 0,85 & 0,79 \\
\hline GO_Est & $28 \%$ & $30 \%$ & 0,93 & 0,90 & $79 \%$ & $82 \%$ & 0,97 & 0,83 & $38 \%$ & $38 \%$ & 1,00 & 1,00 \\
\hline GO_Mun & $45 \%$ & $47 \%$ & 0,96 & 0,92 & $83 \%$ & $85 \%$ & 0,98 & 0,91 & $35 \%$ & $36 \%$ & 0,98 & 0,96 \\
\hline DF_Est & $43 \%$ & $43 \%$ & 1,01 & 1,01 & $80 \%$ & $77 \%$ & 1,03 & 1,16 & $58 \%$ & $61 \%$ & 0,96 & 0,89 \\
\hline
\end{tabular}


Anexo 2: Análise de equidade - pilar tempo de aprendizado

\begin{tabular}{|c|c|c|c|c|c|c|c|c|c|c|c|c|}
\hline \multirow[b]{2}{*}{ UF/Rede } & \multicolumn{4}{|c|}{ Indisciplina } & \multicolumn{4}{|c|}{ Aluno faltam às aulas } & \multicolumn{4}{|c|}{ Atividades de tutoria } \\
\hline & $\begin{array}{l}\text { Grupo } \\
\text { pobre }\end{array}$ & $\begin{array}{l}\text { Demais } \\
\text { grupos }\end{array}$ & $\begin{array}{l}\text { Acesso } \\
\text { relativo }\end{array}$ & $\begin{array}{c}\text { Razão de } \\
\text { chance } \\
\text { (Acesso) }\end{array}$ & $\begin{array}{l}\text { Grupo } \\
\text { pobre }\end{array}$ & $\begin{array}{c}\text { Demais } \\
\text { grupos }\end{array}$ & $\begin{array}{l}\text { Acesso } \\
\text { relativo }\end{array}$ & $\begin{array}{l}\text { Razão de } \\
\text { chance } \\
\text { (Acesso) }\end{array}$ & $\begin{array}{l}\text { Grupo } \\
\text { pobre }\end{array}$ & $\begin{array}{l}\text { Demais } \\
\text { grupos }\end{array}$ & $\begin{array}{l}\text { Acesso } \\
\text { relativo }\end{array}$ & $\begin{array}{c}\text { Razão de } \\
\text { chance } \\
\text { (Acesso) }\end{array}$ \\
\hline Brasil & $48 \%$ & $51 \%$ & 0,93 & 0,87 & $70 \%$ & $69 \%$ & 1,02 & 1,07 & $71 \%$ & $84 \%$ & 0,85 & 0,49 \\
\hline RO_Est & $59 \%$ & $67 \%$ & 0,88 & 0,71 & $73 \%$ & $75 \%$ & 0,98 & 0,93 & $91 \%$ & $93 \%$ & 0,97 & 0,72 \\
\hline RO_Mun & $56 \%$ & $55 \%$ & 1,02 & 1,05 & $55 \%$ & $63 \%$ & 0,86 & 0,70 & $71 \%$ & $82 \%$ & 0,86 & 0,53 \\
\hline AC_Est & $62 \%$ & $69 \%$ & 0,90 & 0,73 & $81 \%$ & $78 \%$ & 1,04 & 1,22 & $76 \%$ & $73 \%$ & 1,04 & 1,17 \\
\hline AC_Mun & $35 \%$ & $44 \%$ & 0,81 & 0,70 & $86 \%$ & $94 \%$ & 0,92 & 0,41 & $82 \%$ & $69 \%$ & 1,19 & 2,01 \\
\hline AM_Est & $31 \%$ & $35 \%$ & 0,88 & 0,83 & $81 \%$ & $80 \%$ & 1,02 & 1,09 & $73 \%$ & $81 \%$ & 0,90 & 0,62 \\
\hline AM_Mun & $30 \%$ & $39 \%$ & 0,76 & 0,66 & $84 \%$ & $90 \%$ & 0,93 & 0,59 & $69 \%$ & $87 \%$ & 0,79 & 0,33 \\
\hline RR_Est & $58 \%$ & $61 \%$ & 0,95 & 0,88 & $74 \%$ & $74 \%$ & 1,00 & 0,99 & $84 \%$ & $91 \%$ & 0,92 & 0,51 \\
\hline RR_Mun & $80 \%$ & $84 \%$ & 0,96 & 0,78 & $100 \%$ & $100 \%$ & 1,00 & 1,00 & $100 \%$ & $100 \%$ & 1,00 & 1,00 \\
\hline PA_Est & $44 \%$ & $43 \%$ & 1,03 & 1,05 & $79 \%$ & $77 \%$ & 1,03 & 1,13 & $41 \%$ & $50 \%$ & 0,82 & 0,69 \\
\hline PA_Mun & $40 \%$ & $43 \%$ & 0,94 & 0,90 & $75 \%$ & $75 \%$ & 0,99 & 0,98 & $61 \%$ & $66 \%$ & 0,92 & 0,80 \\
\hline AP_Est & $45 \%$ & $51 \%$ & 0,89 & 0,80 & $83 \%$ & $82 \%$ & 1,02 & 1,11 & $78 \%$ & $79 \%$ & 0,98 & 0,93 \\
\hline AP_Mun & $56 \%$ & $56 \%$ & 0,99 & 0,99 & $66 \%$ & $63 \%$ & 1,05 & 1,14 & $0 \%$ & $0 \%$ & 0,00 & 0,00 \\
\hline TO_Est & $65 \%$ & $68 \%$ & 0,95 & 0,85 & $77 \%$ & $71 \%$ & 1,09 & 1,38 & $89 \%$ & $91 \%$ & 0,98 & 0,84 \\
\hline TO_Mun & $69 \%$ & $64 \%$ & 1,07 & 1,24 & $67 \%$ & $57 \%$ & 1,17 & 1,51 & $89 \%$ & $92 \%$ & 0,97 & 0,75 \\
\hline MA_Est & $55 \%$ & $58 \%$ & 0,96 & 0,91 & $56 \%$ & $55 \%$ & 1,01 & 1,02 & $46 \%$ & $47 \%$ & 0,97 & 0,95 \\
\hline MA_Mun & $45 \%$ & $49 \%$ & 0,92 & 0,86 & $66 \%$ & $64 \%$ & 1,03 & 1,09 & $53 \%$ & $63 \%$ & 0,85 & 0,68 \\
\hline PI_Est & $56 \%$ & $54 \%$ & 1,04 & 1,10 & $48 \%$ & $50 \%$ & 0,96 & 0,93 & $46 \%$ & $51 \%$ & 0,90 & 0,82 \\
\hline PI_Mun & $44 \%$ & $50 \%$ & 0,89 & 0,80 & $47 \%$ & $50 \%$ & 0,94 & 0,89 & $47 \%$ & $66 \%$ & 0,71 & 0,46 \\
\hline CE_Est & $58 \%$ & $59 \%$ & 0,97 & 0,93 & $86 \%$ & $84 \%$ & 1,02 & 1,14 & $70 \%$ & $69 \%$ & 1,02 & 1,07 \\
\hline CE_Mun & $54 \%$ & $56 \%$ & 0,97 & 0,92 & $63 \%$ & $65 \%$ & 0,97 & 0,91 & $86 \%$ & $87 \%$ & 0,99 & 0,96 \\
\hline RN_Est & $52 \%$ & $58 \%$ & 0,90 & 0,79 & $71 \%$ & $74 \%$ & 0,96 & 0,85 & $49 \%$ & $52 \%$ & 0,93 & 0,87 \\
\hline RN_Mun & $53 \%$ & $57 \%$ & 0,92 & 0,83 & $75 \%$ & $74 \%$ & 1,02 & 1,09 & $52 \%$ & $61 \%$ & 0,86 & 0,70 \\
\hline PB_Est & $47 \%$ & $54 \%$ & 0,87 & 0,76 & $74 \%$ & $71 \%$ & 1,04 & 1,17 & $45 \%$ & $55 \%$ & 0,81 & 0,66 \\
\hline PB_Mun & $49 \%$ & $54 \%$ & 0,91 & 0,83 & $67 \%$ & $63 \%$ & 1,05 & 1,16 & $52 \%$ & $56 \%$ & 0,92 & 0,83 \\
\hline PE_Est & $49 \%$ & $50 \%$ & 0,97 & 0,93 & $76 \%$ & $73 \%$ & 1,03 & 1,13 & $90 \%$ & $88 \%$ & 1,02 & 1,18 \\
\hline PE_Mun & $41 \%$ & $46 \%$ & 0,90 & 0,83 & $70 \%$ & $68 \%$ & 1,02 & 1,07 & $66 \%$ & $67 \%$ & 0,99 & 0,96 \\
\hline AL_Est & $39 \%$ & $44 \%$ & 0,89 & 0,81 & $81 \%$ & $81 \%$ & 1,01 & 1,03 & $44 \%$ & $45 \%$ & 0,98 & 0,97 \\
\hline AL_Mun & $34 \%$ & $34 \%$ & 1,00 & 0,99 & $82 \%$ & $83 \%$ & 0,99 & 0,93 & $69 \%$ & $68 \%$ & 1,02 & 1,07 \\
\hline SE_Est & $48 \%$ & $51 \%$ & 0,94 & 0,88 & $79 \%$ & $78 \%$ & 1,00 & 1,01 & $56 \%$ & $56 \%$ & 0,99 & 0,98 \\
\hline SE_Mun & $40 \%$ & $40 \%$ & 1,00 & 0,99 & $73 \%$ & $72 \%$ & 1,01 & 1,05 & $32 \%$ & $34 \%$ & 0,95 & 0,93 \\
\hline BA_Est & $53 \%$ & $51 \%$ & 1,05 & 1,10 & $75 \%$ & $73 \%$ & 1,03 & 1,12 & $43 \%$ & $52 \%$ & 0,84 & 0,72 \\
\hline BA_Mun & $41 \%$ & $43 \%$ & 0,96 & 0,94 & $72 \%$ & $73 \%$ & 0,99 & 0,95 & $45 \%$ & $51 \%$ & 0,87 & 0,76 \\
\hline MG_Est & $36 \%$ & $39 \%$ & 0,92 & 0,88 & $69 \%$ & $67 \%$ & 1,03 & 1,10 & $86 \%$ & $83 \%$ & 1,03 & 1,22 \\
\hline MG_Mun & $43 \%$ & $51 \%$ & 0,84 & 0,73 & $67 \%$ & $72 \%$ & 0,93 & 0,80 & $85 \%$ & $89 \%$ & 0,96 & 0,74 \\
\hline ES_Est & $46 \%$ & $50 \%$ & 0,92 & 0,86 & $67 \%$ & $64 \%$ & 1,04 & 1,12 & $69 \%$ & $68 \%$ & 1,02 & 1,06 \\
\hline ES_Mun & $51 \%$ & $57 \%$ & 0,89 & 0,78 & $45 \%$ & $46 \%$ & 0,98 & 0,96 & $71 \%$ & $79 \%$ & 0,90 & 0,66 \\
\hline RJ_Est & $48 \%$ & $52 \%$ & 0,92 & 0,85 & $65 \%$ & $63 \%$ & 1,03 & 1,07 & $65 \%$ & $66 \%$ & 0,98 & 0,96 \\
\hline RJ_Mun & $54 \%$ & $55 \%$ & 0,99 & 0,98 & $71 \%$ & $72 \%$ & 0,99 & 0,98 & $87 \%$ & $90 \%$ & 0,97 & 0,80 \\
\hline SP_Est & $48 \%$ & $48 \%$ & 0,98 & 0,97 & $70 \%$ & $68 \%$ & 1,03 & 1,11 & $98 \%$ & $98 \%$ & 1,00 & 0,81 \\
\hline SP_Mun & $44 \%$ & $47 \%$ & 0,94 & 0,89 & $57 \%$ & $53 \%$ & 1,06 & 1,14 & $94 \%$ & $94 \%$ & 1,00 & 0,93 \\
\hline PR_Est & $55 \%$ & $58 \%$ & 0,94 & 0,87 & $75 \%$ & $75 \%$ & 1,00 & 1,01 & $87 \%$ & $92 \%$ & 0,95 & 0,61 \\
\hline PR_Mun & $42 \%$ & $56 \%$ & 0,75 & 0,57 & $68 \%$ & $69 \%$ & 0,98 & 0,95 & $82 \%$ & $85 \%$ & 0,96 & 0,77 \\
\hline SC_Est & $48 \%$ & $53 \%$ & 0,90 & 0,82 & $68 \%$ & $67 \%$ & 1,02 & 1,06 & $27 \%$ & $37 \%$ & 0,73 & 0,63 \\
\hline SC_Mun & $54 \%$ & $57 \%$ & 0,94 & 0,87 & $73 \%$ & $68 \%$ & 1,07 & 1,28 & & & & \\
\hline RS_Est & $57 \%$ & $61 \%$ & 0,94 & 0,86 & $69 \%$ & $65 \%$ & 1,06 & 1,19 & $58 \%$ & $56 \%$ & 1,04 & 1,11 \\
\hline RS_Mun & $58 \%$ & $60 \%$ & 0,97 & 0,93 & $67 \%$ & $63 \%$ & 1,07 & 1,21 & $89 \%$ & $91 \%$ & 0,97 & 0,73 \\
\hline MS_Est & $56 \%$ & $56 \%$ & 1,00 & 1,01 & $74 \%$ & $76 \%$ & 0,98 & 0,92 & $67 \%$ & $63 \%$ & 1,08 & 1,23 \\
\hline MS_Mun & $43 \%$ & $48 \%$ & 0,90 & 0,83 & $72 \%$ & $65 \%$ & 1,09 & 1,33 & $89 \%$ & $92 \%$ & 0,97 & 0,72 \\
\hline MT_Est & $50 \%$ & $52 \%$ & 0,96 & 0,92 & $72 \%$ & $71 \%$ & 1,02 & 1,07 & $97 \%$ & $97 \%$ & 1,01 & 1,22 \\
\hline MT_Mun & $45 \%$ & $54 \%$ & 0,84 & 0,70 & $64 \%$ & $58 \%$ & 1,10 & 1,29 & $88 \%$ & $92 \%$ & 0,95 & 0,62 \\
\hline GO_Est & $50 \%$ & $49 \%$ & 1,00 & 1,00 & $81 \%$ & $79 \%$ & 1,03 & 1,18 & $84 \%$ & $82 \%$ & 1,02 & 1,13 \\
\hline GO_Mun & $45 \%$ & $46 \%$ & 0,99 & 0,97 & $71 \%$ & $70 \%$ & 1,01 & 1,05 & $91 \%$ & $90 \%$ & 1,01 & 1,07 \\
\hline DF_Est & $62 \%$ & $67 \%$ & 0,92 & 0,80 & $79 \%$ & $74 \%$ & 1,08 & 1,36 & $87 \%$ & $84 \%$ & 1,03 & 1,27 \\
\hline
\end{tabular}


Anexo 2: Análise de equidade - pilar recursos escolares

\begin{tabular}{|c|c|c|c|c|c|c|c|c|c|c|c|c|}
\hline \multirow[b]{2}{*}{ UF/Rede } & \multicolumn{4}{|c|}{ Condições de Infraestrutura } & \multicolumn{4}{|c|}{ Equipamentos } & \multicolumn{4}{|c|}{ Infraestrutura } \\
\hline & $\begin{array}{l}\text { Grupo } \\
\text { pobre }\end{array}$ & $\begin{array}{l}\text { Demais } \\
\text { grupos }\end{array}$ & $\begin{array}{l}\text { Acesso } \\
\text { relativo }\end{array}$ & $\begin{array}{c}\text { Razão de } \\
\text { chance } \\
\text { (Acesso) }\end{array}$ & $\begin{array}{l}\text { Grupo } \\
\text { pobre }\end{array}$ & $\begin{array}{c}\text { Demais } \\
\text { grupos }\end{array}$ & $\begin{array}{l}\text { Acesso } \\
\text { relativo }\end{array}$ & $\begin{array}{c}\text { Razão de } \\
\text { chance } \\
\text { (Acesso) }\end{array}$ & $\begin{array}{l}\text { Grupo } \\
\text { pobre }\end{array}$ & $\begin{array}{c}\text { Demais } \\
\text { grupos }\end{array}$ & $\begin{array}{l}\text { Acesso } \\
\text { relativo }\end{array}$ & $\begin{array}{c}\text { Razão de } \\
\text { chance } \\
\text { (Acesso) }\end{array}$ \\
\hline Brasil & $46 \%$ & $54 \%$ & 0,87 & 0,75 & $44 \%$ & $74 \%$ & 0,60 & 0,28 & $23 \%$ & $39 \%$ & 0,60 & 0,48 \\
\hline RO_Est & $43 \%$ & $45 \%$ & 0,97 & 0,94 & $41 \%$ & $46 \%$ & 0,89 & 0,81 & $38 \%$ & $51 \%$ & 0,74 & 0,58 \\
\hline RO_Mun & $28 \%$ & $28 \%$ & 1,02 & 1,02 & $14 \%$ & $25 \%$ & 0,54 & 0,47 & $1 \%$ & $7 \%$ & 0,18 & 0,17 \\
\hline AC_Est & $56 \%$ & $60 \%$ & 0,93 & 0,84 & $30 \%$ & $49 \%$ & 0,61 & 0,45 & $36 \%$ & $56 \%$ & 0,65 & 0,45 \\
\hline AC_Mun & $4 \%$ & $2 \%$ & 2,06 & 2,11 & $2 \%$ & $5 \%$ & 0,50 & 0,49 & $4 \%$ & $2 \%$ & 1,80 & 1,83 \\
\hline AM_Est & $56 \%$ & $61 \%$ & 0,93 & 0,84 & $54 \%$ & $68 \%$ & 0,78 & 0,53 & $31 \%$ & $34 \%$ & 0,92 & 0,88 \\
\hline AM_Mun & $36 \%$ & $43 \%$ & 0,84 & 0,75 & $24 \%$ & $45 \%$ & 0,54 & 0,39 & $13 \%$ & $28 \%$ & 0,46 & 0,38 \\
\hline RR_Est & $33 \%$ & $50 \%$ & 0,67 & 0,50 & $29 \%$ & $52 \%$ & 0,55 & 0,37 & $23 \%$ & $37 \%$ & 0,62 & 0,51 \\
\hline RR_Mun & $68 \%$ & $82 \%$ & 0,83 & 0,47 & $48 \%$ & $65 \%$ & 0,73 & 0,48 & $0 \%$ & $0 \%$ & & \\
\hline PA_Est & $25 \%$ & $40 \%$ & 0,62 & 0,49 & $13 \%$ & $20 \%$ & 0,67 & 0,62 & $23 \%$ & $27 \%$ & 0,86 & 0,82 \\
\hline PA_Mun & $34 \%$ & $32 \%$ & 1,04 & 1,05 & $12 \%$ & $17 \%$ & 0,70 & 0,66 & $6 \%$ & $11 \%$ & 0,53 & 0,50 \\
\hline AP_Est & $42 \%$ & $32 \%$ & 1,30 & 1,53 & $25 \%$ & $31 \%$ & 0,81 & 0,74 & $39 \%$ & $37 \%$ & 1,04 & 1,06 \\
\hline AP_Mun & $42 \%$ & $31 \%$ & 1,35 & 1,60 & $40 \%$ & $60 \%$ & 0,66 & 0,43 & $0 \%$ & $0 \%$ & & \\
\hline TO_Est & $53 \%$ & $59 \%$ & 0,90 & 0,79 & $68 \%$ & $72 \%$ & 0,96 & 0,86 & $46 \%$ & $53 \%$ & 0,87 & 0,75 \\
\hline TO_Mun & $43 \%$ & $63 \%$ & 0,68 & 0,45 & $41 \%$ & $70 \%$ & 0,58 & 0,29 & $9 \%$ & $23 \%$ & 0,38 & 0,32 \\
\hline MA_Est & $24 \%$ & $25 \%$ & 0,97 & 0,96 & $20 \%$ & $28 \%$ & 0,72 & 0,65 & $19 \%$ & $29 \%$ & 0,65 & 0,57 \\
\hline MA_Mun & $37 \%$ & $42 \%$ & 0,87 & 0,79 & $8 \%$ & $19 \%$ & 0,42 & 0,37 & $4 \%$ & $11 \%$ & 0,38 & 0,35 \\
\hline PI_Est & $43 \%$ & $46 \%$ & 0,94 & 0,89 & $24 \%$ & $29 \%$ & 0,84 & 0,79 & $19 \%$ & $26 \%$ & 0,72 & 0,66 \\
\hline PI_Mun & $47 \%$ & $57 \%$ & 0,82 & 0,66 & $19 \%$ & $42 \%$ & 0,46 & 0,33 & $6 \%$ & $14 \%$ & 0,44 & 0,41 \\
\hline CE_Est & $43 \%$ & $49 \%$ & 0,88 & 0,80 & $58 \%$ & $64 \%$ & 0,91 & 0,79 & $60 \%$ & $58 \%$ & 1,02 & 1,06 \\
\hline CE_Mun & $52 \%$ & $54 \%$ & 0,96 & 0,92 & $14 \%$ & $22 \%$ & 0,62 & 0,56 & $10 \%$ & $17 \%$ & 0,59 & 0,54 \\
\hline RN_Est & $48 \%$ & $48 \%$ & 0,99 & 0,99 & $43 \%$ & $46 \%$ & 0,92 & 0,86 & $25 \%$ & $34 \%$ & 0,72 & 0,63 \\
\hline RN_Mun & $43 \%$ & $51 \%$ & 0,84 & 0,72 & $18 \%$ & $35 \%$ & 0,53 & 0,42 & $15 \%$ & $27 \%$ & 0,56 & 0,48 \\
\hline PB_Est & $34 \%$ & $40 \%$ & 0,86 & 0,78 & $20 \%$ & $29 \%$ & 0,69 & 0,61 & $35 \%$ & $42 \%$ & 0,83 & 0,75 \\
\hline PB_Mun & $46 \%$ & $53 \%$ & 0,87 & 0,76 & $14 \%$ & $27 \%$ & 0,52 & 0,44 & $6 \%$ & $11 \%$ & 0,56 & 0,53 \\
\hline PE_Est & $60 \%$ & $58 \%$ & 1,03 & 1,09 & $54 \%$ & $55 \%$ & 0,98 & 0,95 & $34 \%$ & $40 \%$ & 0,86 & 0,79 \\
\hline PE_Mun & $44 \%$ & $47 \%$ & 0,94 & 0,89 & $17 \%$ & $22 \%$ & 0,76 & 0,71 & $5 \%$ & $7 \%$ & 0,80 & 0,79 \\
\hline AL_Est & $37 \%$ & $28 \%$ & 1,32 & 1,51 & $23 \%$ & $30 \%$ & 0,76 & 0,68 & $35 \%$ & $41 \%$ & 0,84 & 0,76 \\
\hline AL_Mun & $26 \%$ & $22 \%$ & 1,17 & 1,23 & $23 \%$ & $24 \%$ & 0,96 & 0,94 & $5 \%$ & $10 \%$ & 0,52 & 0,49 \\
\hline SE_Est & $40 \%$ & $39 \%$ & 1,05 & 1,09 & $59 \%$ & $62 \%$ & 0,96 & 0,90 & $34 \%$ & $43 \%$ & 0,80 & 0,69 \\
\hline SE_Mun & $35 \%$ & $40 \%$ & 0,86 & 0,79 & $15 \%$ & $24 \%$ & 0,63 & 0,56 & $5 \%$ & $15 \%$ & 0,34 & 0,30 \\
\hline BA_Est & $35 \%$ & $33 \%$ & 1,07 & 1,11 & $54 \%$ & $61 \%$ & 0,89 & 0,75 & $28 \%$ & $38 \%$ & 0,74 & 0,64 \\
\hline BA_Mun & $34 \%$ & $42 \%$ & 0,81 & 0,72 & $15 \%$ & $28 \%$ & 0,54 & 0,46 & $5 \%$ & $10 \%$ & 0,50 & 0,47 \\
\hline MG_Est & $50 \%$ & $48 \%$ & 1,03 & 1,06 & $63 \%$ & $76 \%$ & 0,82 & 0,52 & $41 \%$ & $52 \%$ & 0,80 & 0,66 \\
\hline MG_Mun & $53 \%$ & $60 \%$ & 0,88 & 0,75 & $58 \%$ & $83 \%$ & 0,69 & 0,27 & $27 \%$ & $46 \%$ & 0,59 & 0,43 \\
\hline ES_Est & $45 \%$ & $47 \%$ & 0,97 & 0,94 & $78 \%$ & $80 \%$ & 0,97 & 0,88 & $50 \%$ & $54 \%$ & 0,93 & 0,85 \\
\hline ES_Mun & $52 \%$ & $53 \%$ & 0,99 & 0,98 & $67 \%$ & $75 \%$ & 0,89 & 0,66 & $33 \%$ & $46 \%$ & 0,72 & 0,58 \\
\hline RJ_Est & $69 \%$ & $67 \%$ & 1,04 & 1,14 & $91 \%$ & $92 \%$ & 0,99 & 0,89 & $54 \%$ & $58 \%$ & 0,92 & 0,83 \\
\hline RJ_Mun & $57 \%$ & $60 \%$ & 0,95 & 0,88 & $70 \%$ & $80 \%$ & 0,88 & 0,61 & $30 \%$ & $35 \%$ & 0,86 & 0,79 \\
\hline SP_Est & $53 \%$ & $54 \%$ & 0,98 & 0,96 & $82 \%$ & $85 \%$ & 0,97 & 0,85 & $13 \%$ & $17 \%$ & 0,75 & 0,71 \\
\hline SP_Mun & $73 \%$ & $75 \%$ & 0,97 & 0,88 & $87 \%$ & $90 \%$ & 0,97 & 0,73 & $22 \%$ & $27 \%$ & 0,82 & 0,76 \\
\hline PR_Est & $49 \%$ & $51 \%$ & 0,96 & 0,93 & $75 \%$ & $81 \%$ & 0,93 & 0,72 & $64 \%$ & $76 \%$ & 0,84 & 0,56 \\
\hline PR_Mun & $94 \%$ & $86 \%$ & 1,10 & 2,69 & $68 \%$ & $92 \%$ & 0,74 & 0,19 & $44 \%$ & $62 \%$ & 0,71 & 0,48 \\
\hline SC_Est & $48 \%$ & $48 \%$ & 0,99 & 0,98 & $89 \%$ & $91 \%$ & 0,98 & 0,83 & $61 \%$ & $65 \%$ & 0,94 & 0,84 \\
\hline SC_Mun & $57 \%$ & $63 \%$ & 0,90 & 0,78 & $82 \%$ & $88 \%$ & 0,93 & 0,63 & $40 \%$ & $55 \%$ & 0,72 & 0,53 \\
\hline RS_Est & $49 \%$ & $55 \%$ & 0,89 & 0,79 & $92 \%$ & $95 \%$ & 0,97 & 0,61 & $65 \%$ & $70 \%$ & 0,92 & 0,78 \\
\hline RS_Mun & $60 \%$ & $63 \%$ & 0,95 & 0,88 & $75 \%$ & $85 \%$ & 0,89 & 0,56 & $39 \%$ & $51 \%$ & 0,77 & 0,62 \\
\hline MS_Est & $52 \%$ & $51 \%$ & 1,02 & 1,05 & $89 \%$ & $92 \%$ & 0,97 & 0,72 & $63 \%$ & $69 \%$ & 0,91 & 0,75 \\
\hline MS_Mun & $66 \%$ & $77 \%$ & 0,86 & 0,59 & $78 \%$ & $89 \%$ & 0,88 & 0,46 & $50 \%$ & $66 \%$ & 0,76 & 0,52 \\
\hline MT_Est & $63 \%$ & $60 \%$ & 1,05 & 1,13 & $77 \%$ & $83 \%$ & 0,92 & 0,67 & $52 \%$ & $58 \%$ & 0,90 & 0,79 \\
\hline MT_Mun & $37 \%$ & $53 \%$ & 0,70 & 0,52 & $46 \%$ & $63 \%$ & 0,73 & 0,51 & $17 \%$ & $31 \%$ & 0,54 & 0,44 \\
\hline GO_Est & $32 \%$ & $33 \%$ & 0,95 & 0,92 & $29 \%$ & $36 \%$ & 0,79 & 0,71 & $26 \%$ & $35 \%$ & 0,75 & 0,67 \\
\hline GO_Mun & $46 \%$ & $48 \%$ & 0,95 & 0,91 & $45 \%$ & $50 \%$ & 0,89 & 0,81 & $20 \%$ & $26 \%$ & 0,77 & 0,71 \\
\hline DF_Est & $52 \%$ & $46 \%$ & 1,14 & 1,30 & $89 \%$ & $90 \%$ & 0,99 & 0,92 & $68 \%$ & $71 \%$ & 0,96 & 0,87 \\
\hline
\end{tabular}




\title{
Manuscrito 4
}

\section{ESTIMAÇÃO DOS EFEITOS DOS PILARES EDUCACIONAIS SOBRE O DESEMPENHO: UM ESTUDO LONGITUDINAL}

\begin{abstract}
Resumo
Os estudos sobre eficácia escolar têm buscado compreender o papel da escola como pilar da aprendizagem dos alunos e do desempenho de sistemas educacionais. O avanço deste estudo foi estimar os efeitos transversais e longitudinais dos pilares fundamentais da escola definidos pelo modelo de prosperidade educacional sobre o desempenho. $\mathrm{O}$ estudo foi realizado com 5.906 escolas presentes nas edições de 2007, 2009, 2011 e 2013 da Prova Brasil, abrangendo 1.892.024 estudantes. A análise transversal, utilizando somente os dados de 2009, demonstrou o potencial dos pilares educacionais em contribuir com o aprendizado dos alunos. A análise longitudinal demonstrou pequena variabilidade no desempenho ao longo dos anos e essa variabilidade é explicada, sobretudo, pela variância entre escolas $(72 \%)$ e não por mudanças entre aplicações $(28 \%)$. Os resultados, como um todo, indicam pouca mudança no sistema educacional brasileiro, estando esse estagnado.

Palavras-chave: eficácia escolar; desempenho acadêmico; recursos escolares.
\end{abstract}




\begin{abstract}
The studies on school effectiveness have sought to understand the role of the school as a pillar of student learning and performance of educational systems. The advance of the present study was to estimate the transversal and longitudinal effects of the fundamental pillars of the school defined by the educational prosperity model on performance. The study was conducted with 5,906 schools that participated in the 2007, 2009, 2011 and 2013 editions of Prova Brasil, covering 1,892,024 students. Cross-sectional analysis using only the data from 2009 showed the potential of educational pillars in contributing to student learning. Longitudinal analysis showed little variability in performance over the years and this variability is explained mainly by the variance between schools (72\%) and not by changes between applications (28\%). The results as a whole indicate little change in the Brazilian educational system, which itself is stagnant.
\end{abstract}

Keywords: school effectiveness; academic performance; school resources. 
Há várias décadas, pesquisadores têm tentado responder a questões acerca do quanto as escolas podem fazer a diferença no processo educacional e por que algumas escolas fazem mais diferença do que outras. A busca por respostas a esses questionamentos começou ao longo dos anos 1950 e 1960, a partir da disponibilização de dados empíricos provindos de pesquisas educacionais realizadas na Inglaterra, Estados Unidos e França (Forquin, 1995). Essas pesquisas tomaram ainda mais força após a publicação do relatório Coleman (Coleman et al., 1966), o qual indicou um pessimismo pedagógico e levou à conclusão de que as escolas não faziam diferença no desempenho dos estudantes. A repercussão gerada pelas conclusões desse relatório dava início ao campo de pesquisa denominado eficácia escolar.

Mortimore (1991, em Franco et al., 2007) define que escola eficaz é aquela que viabiliza que seus alunos apresentem desempenhos acima do esperado, face à origem social dos alunos e à composição social do corpo discente da escola. Nesse sentido, as investigações na área de eficácia escolar devem buscar compreender, analisar e clarificar o papel da escola como pilar da aprendizagem dos alunos e do desempenho de sistemas educacionais, por meio do efeito escola e da identificação dos fatores que contribuem para que essa escola seja mais eficaz (Ferrão \& Couto, 2013).

Muito se avançou na área de eficácia escolar. Por exemplo, já está confirmada a relação positiva entre o nível socioeconômico e o desempenho acadêmico (Teddlie, Stringfield, \& Reynolds, 2000; White, 1982). Além disso, estudos com amostras de avaliações em larga escala têm apontado que as escolas também variam substancialmente em termos da média de nível socioeconômico dos alunos que recebem. A variação na composição do alunado de uma escola, consequentemente, tem efeitos nas práticas pedagógicas. Escolas que recebem alunos de contextos familiares e sociais mais precários 
enfrentarão dificuldades e percorrerão caminhos diferentes de escolas que recebem alunos mais favorecidos economicamente.

Considerando as indicações de muitas pesquisas estrangeiras e brasileiras que comprovam que a maior parte da variação entre resultados escolares pode ser explicada por fatores extraescolares, estes não podem ser ignorados nos modelos de análise. Depois de controlados os efeitos contextuais - como o nível socioeconômico e o nível de educação dos pais - as pesquisas transversais têm possibilitado a identificação de fatores que tornam algumas escolas mais eficazes do que outras.

Para Willms e Raudenbush (1989) os estudos transversais são importantes por possibilitarem a estimação de dois tipos de efeitos escolares: A e B. O efeito tipo A inclui o efeito de políticas e práticas escolares e efeitos contextuais e sociais sobre o desempenho escolar, sem distingui-los. Ou seja, o efeito tipo A busca explicar o quão bom é o desempenho de um aluno com características médias em uma determinada escola. Já os efeitos tipo B estimam o efeito da escola após o controle de fatores contextuais. Neste caso, busca-se estimar as razões de uma escola ter desempenho relativamente melhor que outra em contextos similares, depois de controlados os efeitos decorrentes de fatores sociais e econômicos que escapam ao controle do sistema escolar.

Revisando a literatura sobre eficácia escolar com dados do Reino Unido, dos Estados Unidos e da Holanda, Sammons, Hillman e Mortimore (1995) chegaram a uma síntese dos fatores mais comumente identificados em escolas eficazes. Esses autores indicam onze características: (1) liderança profissional; (2) objetivos e visões compartilhadas; (3) ambiente de aprendizagem; (4) concentração no ensino e na aprendizagem; (5) ensino e objetivos claros; (6) altas expectativas; (7) incentivo positivo; (8) monitoramento do progresso; (9) direitos e responsabilidades dos alunos; (10) parceria casa-escola; e (11) organização orientada à aprendizagem. 
No Brasil, Franco e Bonamino (2005) indicam que os fatores escolares associados à eficácia escolar comumente encontrados na literatura brasileira podem ser divididos em cinco categorias: (1) recursos escolares; (2) organização e gestão da escola; (3) clima acadêmico; (4) formação e salário docente; e (5) ênfase pedagógica. Um diferencial nos achados brasileiros refere-se ao efeito relevante de infraestrutura e estado de conservação dos equipamentos escolares sobre o desempenho (Albanez, Ferreira, \& Franco, 2002). Esses achados indicam que o sistema educacional brasileiro ainda carece de uma estrutura mínima para o adequado funcionamento da escola, o que não mais ocorre em países desenvolvidos.

Apesar de a maior parte da produção na área se embasar em estudos de delineamento transversal, existe um consenso de que o melhor delineamento para o estudo de eficácia escolar é o longitudinal (Willms \& Raudenbush, 1989; Timmermans, Snijders, \& Bosker, 2012). Em estudos transversais, a medida de desempenho representa o agregado de todo o aprendizado adquirido pelo estudante ao longo dos anos escolares e não o valor agregado pela escola (Franco, Brooke, \& Alves, 2008). Assim, a existência de mais de uma medida de desempenho do aluno permite corrigir o descompasso entre a medida de output e a medida das condições escolares, possibilitando a melhor estimação do efeito-escola.

Ademais, os estudos longitudinais permitem que o efeito-escola estimado seja composto por um efeito médio dentro de um determinado período, componente estável, e um efeito específico de cada período de avaliação, componente instável (Willms \& Raudenbush, 1989). Por outro lado, apesar de ser o delineamento mais desejado, é também um delineamento difícil de ser implementado e que demanda recursos consideráveis. Essas dificuldades operacionais relacionadas a esse tipo de delineamento acabam por restringir a quantidade de grupos de pesquisa que possuem condições de executá-las. 
Dado esse contexto, o principal objetivo deste estudo é contribuir para o avanço dos estudos longitudinais que buscam melhor compreender a estabilidade do efeito escola no decorrer do tempo. A principal fonte de dados para a realização de estudos na área de eficácia escolar no Brasil tem sido o Sistema de Avaliação da Educação Básica (SAEB). Trata-se de uma avaliação seccional que, de dois em dois anos, levanta informações sobre alunos e escolas (Franco, Brooke, \& Alves, 2008). Em 2005, o Governo Federal realizou alterações no SAEB, que passou a ser composto por um segmento amostral e um segmento censitário, este último englobando todas as escolas públicas brasileiras com mais de 20 alunos matriculados na série avaliada (Brasil, 2005). Esse segmento censitário é comumente denominado de Prova Brasil e foi implantado com o intuito garantir um melhor monitoramento das escolas públicas, definindo metas individuais para todas.

Apesar de ainda não permitir um delineamento longitudinal ${ }^{1}$ que acompanha o desempenho de cada estudante, a Prova Brasil possibilitou o delineamento de estudos que buscam acompanhar o desempenho das escolas ao longo dos anos. Tendo essa perspectiva, um diferencial do presente trabalho é acompanhar longitudinalmente o desempenho das escolas públicas brasileiras, utilizando como controle características contextuais, distinguindo-se dos tradicionais estudos multiníveis que utilizam o estudante no nível 1 e a escola no nível 2.

Para tanto, será feito uso de um modelo multinível de medidas repetidas por escola. O modelo de medidas repetidas foi desenvolvido para atender estudos de delineamento longitudinal em que o primeiro nível (intrassujeito) consiste nas medidas repetidas do desempenho dos estudantes e o segundo nível diz respeito às variáveis do estudante mantidas constantes (Hox, 2010). De modo semelhante, esse modelo pode ser empregado

\footnotetext{
${ }^{1}$ Desde 2007 o INEP introduziu um indicador único por aluno no levantamento censitário anual. Esse procedimento tornou possível a execução de estudos longitudinais no nível do aluno. Contudo, até o período de execução das análises deste estudo poucos alunos poderiam ser monitorados, dado que a Prova Brasil ocorre de dois em dois anos e é aplicada somente no $5^{\circ}$ e $9^{\circ}$ ano do Ensino Fundamental.
} 
para medidas repetidas de cortes transversais (DiPrete \& Grusky, 1990; Raudenbush \& Chan, 1993).

No caso deste estudo, têm-se medidas repetidas em cortes transversais das escolas brasileiras, ou seja, as mesmas escolas são avaliadas em diferentes períodos, mas com grupos diferentes de estudantes. Com isso, a série de medidas repetidas da escola passa a compor o nível mais baixo e o nível mais alto é composto por variáveis da escola mantidas constantes. Ao empregar esse tipo de análise, espera-se diferenciar o quanto a evolução do desempenho das escolas está relacionada a mudanças nos fatores contextuais e nos fatores escolares.

Outro diferencial deste estudo é o uso de um modelo teórico emergente como base. A partir dos resultados de vários estudos na área de eficácia escolar, Willms (2015) propõe o modelo de prosperidade educacional que busca contemplar os elementos-chave que subsidiam o sucesso de um sistema educacional, sendo que o termo sucesso busca contemplar aspectos cognitivos, sociais, emocional e físico. Para Willms, a garantia do sucesso perpassa três estruturas fundamentais: a família, a instituição (escola) e a comunidade. Como o modelo também se embasa nos estágios desenvolvimentais, são definidos pilares específicos, essenciais para garantir a concretude dos principais resultados esperados para cada etapa educacional.

Esse modelo representa um importante avanço para a área. Primeiro por contribuir para tornar a área mais teoricamente direcionada. Por vezes, os estudos sobre eficácia escolar foram criticados por ser um conjunto de estudos empíricos precários de fundamentação teórica (Van Den Eeden, Hox, \& Hauer, 1990). Segundo porque a definição de pilares e a construção de medidas para esses pilares vêm a contribuir na síntese e no monitoramento dos sistemas educacionais, conforme apresentado por Karino (2016a). 
Neste estudo, tem-se como interesse específico analisar o efeito de variáveis escolares sobre o desempenho médio das escolas em matemática no final do ensino fundamental. Para esse estágio do desenvolvimento, o modelo define quatro pilares como fundamentais: (1) qualidade do ensino; (2) ambiente inclusivo; (3) tempo de aprendizado; e (4) recursos escolares. Esses pilares já foram abordados em estudos anteriores (Karino, 2016a; Karino, 2016b). Em Karino (2016a), buscou-se desenvolver medidas para os quatro pilares do modelo de prosperidade educacional utilizando os questionários contextuais da Prova Brasil. Essas medidas serão base para a presente análise.

Em suma, esta pesquisa, utilizando a escola como unidade e a perspectiva do modelo de prosperidade educacional, busca analisar o efeito de características escolares sobre o desempenho e a estabilidade desses efeitos ao longo do tempo. Primeiramente, utilizando os dados de 2009, busca-se verificar os efeitos dos pilares escolares sobre o desempenho dos estudantes. Em seguida, ao se utilizar uma análise longitudinal de cortes transversais, espera-se compreender o aumento no desempenho escolar nos últimos anos, distinguindo melhor as mudanças de desempenho decorrentes de mudanças contextuais, daquelas provenientes de fatores intrinsecamente escolares. De modo geral, tem-se como objetivos específicos: (1) identificar o efeito dos pilares da escola sobre o desempenho; (2) identificar a variação de desempenho das escolas entre 2007 e 2013; (3) estimar o efeito de medidas repetidas de nível socioeconômico e escolares sobre o desempenho; e (4) identificar os sistemas de ensino (unidades da federação) mais eficazes no Brasil. 


\section{Método}

\section{Participantes}

Este estudo utiliza a base de dados de Avaliação Nacional de Rendimento Escolar (ANRESC ou Prova Brasil) e a base de dados do Censo da Educação Básica dos anos 2007, 2009, 2011 e 2013, ambas disponibilizadas pelo Instituto Nacional de Estudos e Pesquisas Educacionais (Inep). Não foram utilizadas as edições 2005 (primeiro ano de aplicação da prova) e 2015 porque os microdados não tinham sido disponibilizados pelo Inep até o momento de execução da pesquisa.

O público selecionado foi os estudantes do $9^{\circ}$ ano do Ensino Fundamental. Para a análise transversal dos dados, foi selecionada uma amostra composta por 862.114 estudantes provindos de 14.730 escolas da edição de 2009. Para a análise longitudinal das escolas, utilizou-se uma amostra de 5.906 escolas presentes nas quatro edições mencionadas da Prova Brasil, abrangendo 1.892.024 estudantes.

\section{Instrumentos/Variáveis}

As medidas utilizadas neste estudo foram desenvolvidas pelo próprio Inep ou em estudos anteriores com base nas provas e questionários aplicados na Prova Brasil. A Tabela 1 apresenta a descrição da variável dependente - desempenho em matemática - utilizada como indicadora dos resultados alcançados pelos alunos, além das medidas de nível socioeconômico e suporte familiar e da comunidade, apontadas nos estudos de eficácia escolar como importantes indicadores contextuais. 
Tabela 1

Descrição das variáveis: desempenho escolar, suporte familiar/comunidade e nível socioeconômico

\begin{tabular}{|c|c|c|}
\hline Variável & Instrumento & Descrição \\
\hline Proficiência em matemática & $\begin{array}{l}\text { Prova de } \\
\text { Matemática } \\
\text { (Prova Brasil) }\end{array}$ & $\begin{array}{l}\text { Variável escalar desenvolvida pelo Inep com base } \\
\text { na teoria de resposta ao item (TRI) para permitir } \\
\text { monitoramento da educação básica. A escala possui } \\
\text { média } 250 \text { e desvio padrão } 50 \text {. }\end{array}$ \\
\hline Pai e mãe lendo & $\begin{array}{l}\text { Questionário do } \\
\text { aluno }\end{array}$ & $\begin{array}{l}\text { Variável dicotômica que pergunta se o estudante vê } \\
\text { sua mãe ou seu pai lendo }(0=\text { não, } 1=\operatorname{sim}) \text {. }\end{array}$ \\
\hline Livros & $\begin{array}{l}\text { Questionário do } \\
\text { aluno }\end{array}$ & $\begin{array}{l}\text { Pergunta sobre a quantidade de livros que o } \\
\text { estudante possui em casa: (0) nenhum; (1) } 1 \text { a } 20 \\
\text { livros; (2) } 20 \text { a } 100 \text { livros e (3) mais de } 100 \text { livros. }\end{array}$ \\
\hline Suporte familiar & $\begin{array}{l}\text { Questionário do } \\
\text { aluno }\end{array}$ & $\begin{array}{l}\text { Escala desenvolvida por Karino (2016a) composta } \\
\text { por } 6 \text { perguntas que avaliam o envolvimento dos } \\
\text { pais nas atividades escolares, como: ir a reuniões, } \\
\text { incentivar os filhos a estudar etc. A intepretação da } \\
\text { escala é dividida em } 3 \text { níveis. }\end{array}$ \\
\hline Suporte da comunidade & $\begin{array}{l}\text { Questionário do } \\
\text { aluno }\end{array}$ & $\begin{array}{l}\text { Escala desenvolvida por Karino (2016a) composta } \\
\text { por } 4 \text { perguntas que avaliam o grau em que a } \\
\text { comunidade fornece suporte à escola, por exemplo, } \\
\text { promovendo campanhas e ajudando na sua } \\
\text { manutenção. A intepretação da escala é dividida em } \\
3 \text { níveis. }\end{array}$ \\
\hline $\begin{array}{l}\text { Nível socioeconômico } \\
\text { (NSE) }\end{array}$ & $\begin{array}{l}\text { Questionário do } \\
\text { aluno }\end{array}$ & $\begin{array}{l}\text { Escala desenvolvida por Karino (2016a) composta } \\
\text { por } 12 \text { perguntas que abordam a presença de } \\
\text { empregada doméstica em casa, a escolaridade dos } \\
\text { pais e a posse de bens domésticos. A interpretação } \\
\text { da escala é dividida em } 5 \text { níveis. }\end{array}$ \\
\hline
\end{tabular}

Na Tabela 2, são descritas as variáveis dos pilares 1 e 2 (qualidade do ensino e ambiente inclusivo) do modelo de prosperidade educacional. 
Tabela 2

Descrição das variáveis representantes dos pilares 1 e 2 do modelo de prosperidade educacional

\begin{tabular}{|c|c|c|}
\hline Variável & Instrumento & Descrição \\
\hline $\begin{array}{l}\text { Professor corrige dever de } \\
\text { casa - indicador do pilar } \\
\text { qualidade do ensino }\end{array}$ & $\begin{array}{l}\text { Questionário } \\
\text { do aluno }\end{array}$ & $\begin{array}{l}\text { Pergunta sobre a frequência com que o professor } \\
\text { corrige o dever de casa de matemática, com três opções } \\
\text { de resposta: (1) nunca ou quase nuca; (2) de vez em } \\
\text { quando; e (3) sempre ou quase sempre. Quando } \\
\text { utilizada no nível da escola, essa variável foi } \\
\text { transformada para o percentual de alunos que indicam } \\
\text { que o professor sempre ou quase sempre corrige dever } \\
\text { de casa, categorizado em (1) menos de } 80 \% \text { dos alunos, } \\
\text { (2) entre } 80 \text { a } 90 \% \text { dos alunos e (3) acima de } 90 \% \text {. }\end{array}$ \\
\hline $\begin{array}{l}\text { Atividades extras (esporte e } \\
\text { artes) - indicador do pilar } \\
\text { qualidade do ensino }\end{array}$ & ário & $\begin{array}{l}\text { Verifica se a escola oferece como atividades extras } \\
\text { aulas de esporte e artes: (0) não ou (1) sim. }\end{array}$ \\
\hline $\begin{array}{l}\text { Escolaridade do diretor - } \\
\text { indicador do pilar qualidade } \\
\text { do ensino }\end{array}$ & $\begin{array}{l}\text { nário } \\
\text { or }\end{array}$ & $\begin{array}{l}\text { Nível de escolaridade do diretor: (1) menos que o } \\
\text { ensino médio; (2) ensino médio, (3) ensino superior; } \\
\text { (4) ensino superior + atualização; (5) ensino superior + } \\
\text { especialização; (6) ensino superior + mestrado ou } \\
\text { doutorado. }\end{array}$ \\
\hline $\begin{array}{l}\text { Escolaridade do professor - } \\
\text { indicador do pilar qualidade } \\
\text { do ensino }\end{array}$ & $\begin{array}{l}\text { Que } \\
\text { do p }\end{array}$ & $\begin{array}{l}\text { Média de escolaridade dos professores da escola } \\
\text { seguindo a mesma categorização utilizada para Diretor. }\end{array}$ \\
\hline $\begin{array}{l}\text { Adequação do currículo - } \\
\text { indicador do pilar qualidade } \\
\text { do ensino }\end{array}$ & $\begin{array}{l}\text { Que } \\
\text { do } p\end{array}$ & $\begin{array}{l}\text { Percentual de professores da escola que avaliam o } \\
\text { currículo como adequado. }\end{array}$ \\
\hline $\begin{array}{l}\text { \% de cumprimento do } \\
\text { currículo - indicador do } \\
\text { pilar qualidade do ensino }\end{array}$ & $\begin{array}{l}\text { Que } \\
\text { do } p\end{array}$ & $\begin{array}{l}\text { Percentual de professores da escola que afirmam ter } \\
\text { cumprido } 80 \% \text { do currículo. }\end{array}$ \\
\hline $\begin{array}{l}\text { Práticas pedagógicas - } \\
\text { indicador do pilar qualidade } \\
\text { do ensino }\end{array}$ & do profe & $\begin{array}{l}\text { Escala desenvolvida por Karino (2016a) composta por } \\
5 \text { perguntas que avaliam a ocorrência de algumas } \\
\text { atividades pedagógicas. Ela possui três níveis. A cada } \\
\text { nível mais atividades são executadas. }\end{array}$ \\
\hline $\begin{array}{l}\text { Material didático - indicador } \\
\text { do pilar qualidade do ensino }\end{array}$ & $\begin{array}{l}\text { Questionário } \\
\text { do professor }\end{array}$ & $\begin{array}{l}\text { Verifica a proporção de alunos que possui material } \\
\text { didático na escola: (0) nenhum; (1) menos da metade; } \\
\text { (2) metade; (3) a maioria e (4) todos. }\end{array}$ \\
\hline $\begin{array}{l}\text { Material didático em tempo } \\
\text { - indicador do pilar } \\
\text { qualidade do ensino }\end{array}$ & $\begin{array}{l}\text { Questionário } \\
\text { do professor }\end{array}$ & $\begin{array}{l}\text { Verifica se o material didático chegou à escola em } \\
\text { tempo hábil para início do ano letivo: (0) não ou (1) } \\
\text { sim. }\end{array}$ \\
\hline $\begin{array}{l}\text { Qualidade do material } \\
\text { didático - indicador do pilar } \\
\text { qualidade do ensino }\end{array}$ & $\begin{array}{l}\text { Questionário } \\
\text { do professor }\end{array}$ & $\begin{array}{l}\text { Verifica a avaliação do professor quanto ao material } \\
\text { didático da escola: (0) não se aplica; (1) ruim; (2) } \\
\text { razoável; (3) bom e (4) adequado. }\end{array}$ \\
\hline $\begin{array}{l}\text { Teste de seleção - indicador } \\
\text { negativo do pilar ambiente } \\
\text { inclusivo }\end{array}$ & $\begin{array}{l}\text { Questionário } \\
\text { do diretor }\end{array}$ & $\begin{array}{l}\text { Verifica se a escola realiza teste de seleção para } \\
\text { ingresso dos estudantes na escola: }(0) \text { não ou (1) sim. }\end{array}$ \\
\hline
\end{tabular}

Na Tabela 3, são descritas as variáveis dos pilares 3 e 4 (tempo de aprendizado e recursos escolares) do modelo de prosperidade educacional. Apresenta-se também a escala de infraestrutura desenvolvida com base no questionário do censo escolar, levantamento de 
dados estatísticos educacionais de âmbito nacional realizado todos os anos e coordenado

pelo Inep. Essa variável foi incluída no estudo somente na análise longitudinal, após

verificação de que a escala de condições de infraestrutura, desenvolvida com base no

questionário da Prova Brasil, não apresentou efeito significativo sobre o desempenho,

como será posteriormente melhor detalhado.

Tabela 3

Descrição das variáveis representantes dos pilares 3 e 4 do modelo de prosperidade educacional

\begin{tabular}{|c|c|c|}
\hline Variável & Instrumento & Descrição \\
\hline $\begin{array}{l}\text { Indisciplina - indicador } \\
\text { negativo do pilar tempo de } \\
\text { aprendizado }\end{array}$ & $\begin{array}{l}\text { Questionário } \\
\text { do professor }\end{array}$ & $\begin{array}{l}\text { Percentual de professores que avaliam que a } \\
\text { indisciplina dos estudantes é um problema na escola. }\end{array}$ \\
\hline $\begin{array}{l}\text { Alunos faltam às aulas - } \\
\text { indicador negativo do pilar } \\
\text { tempo de aprendizado }\end{array}$ & $\begin{array}{l}\text { Questionário } \\
\text { do professor }\end{array}$ & $\begin{array}{l}\text { Percepção dos professores de que o alto índice de falta } \\
\text { dos alunos é problema na escola: (0) não é um } \\
\text { problema; (1) é um problema, mas não grave e (3) é } \\
\text { um problema grave. }\end{array}$ \\
\hline $\begin{array}{l}\text { Professores faltam às aulas - } \\
\text { indicador negativo do pilar } \\
\text { tempo de aprendizado }\end{array}$ & $\begin{array}{l}\text { ário } \\
\text { sor }\end{array}$ & $\begin{array}{l}\text { Percepção dos professores de que o alto índice de falta } \\
\text { dos professores é problema na escola: (0) não é um } \\
\text { problema; (1) é um problema, mas não grave e (3) é } \\
\text { um problema grave. }\end{array}$ \\
\hline $\begin{array}{l}\text { Atividades de tutoria - } \\
\text { indicador do pilar tempo de } \\
\text { aprendizado }\end{array}$ & ário & $\begin{array}{l}\text { Verifica se a escola oferece atividades de tutoria: (0) } \\
\text { não ou (1) sim. }\end{array}$ \\
\hline $\begin{array}{l}\text { Escala de Recursos } \\
\text { Humanos - indicador do } \\
\text { pilar recursos da escola }\end{array}$ & $\begin{array}{l}\text { hário } \\
\text { or }\end{array}$ & $\begin{array}{l}\text { Escala desenvolvida por Karino (2016a) composta por } \\
4 \text { perguntas que avaliam a percepção do diretor acerca } \\
\text { da presença de problemas de recursos humanos na } \\
\text { escola. A interpretação da escala é dividida em } 3 \\
\text { níveis. }\end{array}$ \\
\hline $\begin{array}{l}\text { Escala de condições de } \\
\text { infraestrutura - indicador do } \\
\text { pilar recursos da escola }\end{array}$ & $\begin{array}{l}\text { Questionário } \\
\text { da escola }\end{array}$ & $\begin{array}{l}\text { Escala desenvolvida por Karino (2016a) composta por } \\
11 \text { perguntas que abordam as condições de } \\
\text { infraestrutura da escola. A interpretação da escala é } \\
\text { dividida em } 4 \text { níveis. }\end{array}$ \\
\hline $\begin{array}{l}\text { Escala de equipamentos - } \\
\text { indicador do pilar recursos } \\
\text { da escola }\end{array}$ & $\begin{array}{l}\text { Questionário } \\
\text { da escola }\end{array}$ & $\begin{array}{l}\text { Escala desenvolvida por Karino (2016a) composta por } \\
8 \text { perguntas que abordam a presença de equipamentos } \\
\text { na escola. A interpretação da escala é dividida em } 4 \\
\text { níveis. }\end{array}$ \\
\hline Escala de infraest & $\begin{array}{l}\text { Censo da } \\
\text { Educação } \\
\text { Básica }\end{array}$ & $\begin{array}{l}\text { Escala desenvolvida por Soares Neto, Jesus, Karino e } \\
\text { Andrade (2013) composta por } 22 \text { perguntas que } \\
\text { avaliam a existência de determinados itens de } \\
\text { infraestrutura na escola. A escala é dividida em } 6 \\
\text { níveis. }\end{array}$ \\
\hline
\end{tabular}

Além dessas variáveis, utilizou-se também a indicação de Unidade da Federação, rede administrativa das escolas e tempo (ocasião de aplicação da Prova Brasil: 2007 = 0 , $2009=1,2011=2,2013=3)$. 


\section{Procedimentos}

Considerando que no modelo multinível os casos com dados ausentes são excluídos no decorrer das etapas de análises, optou-se pelo método listwise deletion para manter o mesmo tamanho amostral ao longo de todo o processo. Para a análise transversal, a mostra deste estudo representa pouco mais de $40 \%$ da base inicial disponibilizada pelo Inep. Para a análise longitudinal, o método listwise deletion resultou em uma perda amostral maior, com o aproveitamento um pouco maior que $16 \%$ da base composta por todas as escolas participantes das quatro edições utilizadas neste estudo. Vale destacar que a abstenção de respostas aos questionários é bastante alta, como já apontado em outros estudos (Karino, Vinha, \& Laros, 2014), o que resulta em uma perda amostral alta, mesmo utilizando algum tratamento para os dados omissos. Além disso, o método listwise deletion demonstrou bons resultados quando comparado a outros métodos mais complexos (Vinha, 2016).

Uma vez consistidas e agregadas as bases de dados, prosseguiu-se para a análise transversal de regressão multinível, na qual dois níveis foram considerados: nível 1 - aluno e nível 2 - escola. As análises foram executadas utilizando o programa HLM 7.0 (Raudenbush, Bryk, Cheong, Congdon, \& du Toit, 2011). O método utilizado para inserção das variáveis no modelo teve como referência o proposto por Hox (2010), composto por cinco etapas:

1. Análise do modelo sem nenhuma variável explicativa - modelo nulo. Este modelo serve para a comparação com os modelos subsequentes e para o cálculo da correlação intraclasse.

2. Inserção no modelo das variáveis explicativas fixas do nível mais baixo (aluno). Primeiro introduziu-se a variável nível socioeconômico e depois as variáveis de suporte familiar/comunidade. 
3. Inserção no modelo das variáveis explicativas fixas do nível mais alto (escola). Nessa etapa, as variáveis referentes a cada um dos pilares foram inseridas gradativamente (primeiro pilar 1, depois o 2, e assim sucessivamente) a fim verificar o impacto de cada um dos pilares.

4. Avaliação da existência de coeficientes de inclinação das variáveis explicativas do nível 1 que possuem componente de variância significativo entre as escolas.

5. Avaliação das relações de interação entre níveis (efeito cross-level): relação entre variáveis explicativas do nível da escola e aquelas do nível do aluno.

Foram mantidas nas análises somente as variáveis que apresentaram significância estatística (Z test: $p$-value $<0,001$ e Razão $t<2$ ) (Raudenbush \& Bryk, 2002). Também foi observado o valor de deviance, estimativa de desajuste do modelo. Utilizando-se de um Teste $\chi^{2}$, foi verificado se houve uma diminuição significativa no valor de uma etapa para outra, indicando um melhor ajuste do modelo (Bickel, 2007; Kreft \& De Leeuw, 1998). Como os testes de significância tendem a apontar efeitos significativos em amostras muito grandes, mesmo quando as mudanças são pequenas, buscou-se aumentar o rigor, adicionando-se o critério de possuir efeito (coeficiente de regressão) maior do que 1. Uma vez que a variável foi incluída no modelo, não mais a retirávamos, mesmo que seu efeito deixasse de ser significativo ou seu coeficiente de regressão viesse a diminuir. Por fim, optou-se pela utilização das variáveis não padronizadas, a fim de se manter as interpretações das escalas originais.

Em seguida, executou-se a análise longitudinal das escolas, considerando somente as variáveis com efeitos significativos na análise transversal. Neste novo modelo, o nível 1 contempla as medidas repetidas da escolas realizadas ao longo das edições da Prova Brasil (variância ao longo do tempo - intraindivíduo) e o nível 2 contempla a escola com suas variáveis fixas (variância entre indivíduos, no caso escolas). O uso do modelo longitudinal 
de dados de corte transversais foi utilizado por DiPrete e Grusky (1990) e Raudenbush e Chan (1993). Nesse modelo, as escolas são acompanhadas ao longo dos anos, sendo que o conjunto de alunos avaliados em cada ano é diferente.

Por fim, o método utilizado para inserção das variáveis no modelo segue o proposto por Hox (2010):

1. Análise do modelo sem nenhuma variável explicativa - modelo nulo.

2. Inclusão da variável relativa ao tempo (ocasião). Essa variável assume os valores 0, 1, 2 e 3, para as avaliações de 2007, 2009, 2011 e 2013, respectivamente. Nesse passo, supõe-se que a taxa de aprendizado tem efeito fixo.

3. Inclusão das variáveis explicativas da escola que podem variar de uma ocasião para outra.

4. Inclusão das variáveis explicativas da escola, invariantes no tempo.

5. Avaliação da existência de coeficientes de inclinação das variáveis do nível 1 que possuem componente de variância significativo entre as escolas.

6. Avaliação das relações de interação entre níveis (efeito cross-level): relação entre variáveis relativas ao tempo (ocasião) e a escola.

\section{Resultados e Discussão}

A análise do modelo nulo (Modelo 0) é importante para se ter uma estimação de linha de base da variância (Tabela 4). Dada a variância inicial, é possível calcular o percentual de variância explicada com a inclusão no modelo das variáveis explicativas. No modelo nulo, verificou-se um intercepto de 240,2, o qual representa o valor médio da proficiência em matemática dos alunos do $9^{\circ}$ ano do ensino fundamental em 2009. É com base nesse modelo também que se calcula a correlação intraclasse (intraclass correlation coefficient - 
ICC), no caso, 0,18. Esse coeficiente pode variar entre 0 e 1 e indica a necessidade de se utilizar a análise multinível. Há autores que consideram que quando esse valor é superior a 10\%, como é o caso, justifica-se a utilização do modelo multinível (Ferrão, 2003; Lee, 2008). Todavia, dependendo das circunstâncias, mesmo valores de ICC entre 5\% e 10\% já são indicativos de uma dependência entre níveis (Bickel, 2007).

$\mathrm{Na}$ etapa seguinte, foram inseridas as variáveis do nível 1. Primeiro, inclui-se a variável relativa à condição socioeconômica do estudante (modelo 1), com a inclusão dessa variável $0,8 \%$ e $16,8 \%$ da variância do nível 1 e 2 foi explicada, respectivamente. O efeito observado dessa variável indica que a cada elevação de nível na escala socioeconômica, aumentam-se em média 5 pontos na escala de matemática. Esse resultado corrobora estudos anteriores que apontam a forte relação entre nível socioeconômico e desempenho acadêmico (Soares, 2004; Teddlie, Stringfield, \& Reynolds, 2000; White, 1982).

Muitos estudos apontam que o efeito do capital socioeconômico, quando agregado para o nível da escola, é ainda maior, com efeitos entre 20 e 58 pontos na escala de proficiência (Andrade \& Laros, 2007; Jesus \& Laros, 2004; Laros, Marciano, \& Andrade, 2010). Destaca-se, no entanto, que esses estudos foram realizados utilizando uma amostra composta por escolas públicas e privadas, o que aumenta a variabilidade tanto no nível socioeconômico como no desempenho acadêmico. Num estudo realizado com os dados da Prova Brasil 2009, contemplando somente escolas públicas, observou-se um efeito de 9,3 pontos (Vinha, Karino, \& Laros, 2016). No presente estudo, quando agregamos o nível socioeconômico dos alunos para o nível da escola, obteve-se um efeito de 14,5 pontos na escala de proficiência a cada elevação de nível na escala socioeconômica, valor semelhante ao estudo de Vinha, Karino e Laros (2016). Além disso, o percentual de variância explicada do nível 2 aumenta para 38,2\%, diferença de 21,4 pontos percentuais. 
Entretanto, após a inclusão de todas as variáveis no modelo, sobretudo as referentes ao nível 2, a diferença do percentual de variância explicada final do nível 2, comparando os modelos com e sem variável nível socioeconômico agregada é de 9,5 pontos. Considerando ainda as evidências de problemas de equidade do sistema educacional brasileiro (Karino, 2016b), em que estudantes com melhores condições socioeconômica tendem a estar em escolas mais bem equipadas e estruturadas, tem-se a hipótese de que parte da variância explicada no nível 2 pela agregação do nível socioeconômico dos alunos está relacionada a diferenças nas condições escolares. Essa hipótese também é corroborada pelo valor alto de correlação do nível socioeconômico agregado com variáveis do nível da escola, por exemplo, equipamentos e infraestrutura.

Quando não é realizado o controle pelo nível socioeconômico nas análises de regressão multinível, pode-se atribuir à escola efeitos relativos ao contexto sociocultural do aluno (Soares, Alves, \& Oliveira, 2001; Willms, 1992). Quando, adicionalmente, se utiliza o nível socioeconômico agregado nas análises, busca-se especificamente estimar o efeito de composição das escolas (Willms, 2010). Por outro lado, ao se agregar o nível socioeconômico do aluno para o nível da escola, pode-se também estar atribuindo ao nível socioeconômico o efeito relativo às diferenças escolares e aos problemas de equidade do sistema educacional (Fletcher, 1998). Essa é uma discussão extremamente complicada, uma vez que há uma tendência natural de multicolinearidade dos dados.

Considerando que nosso interesse é compreender melhor o efeito de variáveis escolares sobre o desempenho, optou-se por retirar o nível socioeconômico agregado das análises a fim de melhor explicitar os efeitos de variáveis escolares. Uma vantagem adicional dessa decisão é que variáveis escolares representam práticas sob controle da própria escola, podendo ser alteradas, enquanto a mudança no nível socioeconômico dos alunos de uma escola exige medidas para além do esforço educacional. 
Dando prosseguimento, quando acrescentadas as variáveis de suporte familiar e da comunidade, duas variáveis não apresentaram efeitos significativos e foram retiradas do modelo: suporte da comunidade e ver o pai lendo. O modelo 2 (Tabela 4) é composto pelo nível socioeconômico e pelas variáveis de suporte com efeito significativo, ele apresenta um percentual de variância explicada um pouco menor no nível do 2 do que o já relatado quando somente o nível socioeconômico tinha sido incluído no modelo.

Tabela 4

Modelos 0,1 e 2 da análise multinível - Prova Brasil 2009

\begin{tabular}{|c|c|c|c|c|c|c|c|}
\hline \multirow{2}{*}{$\begin{array}{c}\text { Fator/ } \\
\text { Pilar }\end{array}$} & \multirow[b]{2}{*}{ Efeito fixo } & \multicolumn{2}{|c|}{ Modelo 0} & \multicolumn{2}{|c|}{ Modelo 1} & \multicolumn{2}{|c|}{ Modelo 2} \\
\hline & & Efeito & $\mathrm{EP}$ & Efeito & $\mathrm{EP}$ & Efeito & $\mathrm{EP}$ \\
\hline & Intercepto & 240,2 & 0,17 & 229,8 & 0,19 & 229,2 & 0,32 \\
\hline Controle & NSE & & & 5,01 & 0,01 & 4,43 & 0,06 \\
\hline \multirow{9}{*}{$\begin{array}{l}\text { Suporte } \\
\text { Familiar }\end{array}$} & Ver mãe lendo & & & & & 1,28 & 0,13 \\
\hline & Número de livros & & & & & 4,32 & 0,07 \\
\hline & Suporte familiar & & & & & $-1,49$ & 0,10 \\
\hline & \multicolumn{7}{|l|}{ Efeito aleatório } \\
\hline & Nível 1 - aluno & \multicolumn{2}{|c|}{$1.706,9$} & \multicolumn{2}{|c|}{$1.693,0$} & \multicolumn{2}{|c|}{$1.683,3$} \\
\hline & Nível 2 - escola & \multicolumn{2}{|c|}{371,0} & \multicolumn{2}{|c|}{308,8} & \multicolumn{2}{|c|}{314,1} \\
\hline & \multicolumn{7}{|l|}{ Variância Explicada } \\
\hline & \multicolumn{3}{|l|}{ Nível 1 - aluno } & \multicolumn{2}{|c|}{$0,8 \%$} & \multicolumn{2}{|c|}{$1,4 \%$} \\
\hline & \multicolumn{3}{|l|}{ Nível 2 - escola } & \multicolumn{2}{|c|}{$16,8 \%$} & \multicolumn{2}{|c|}{$15,3 \%$} \\
\hline
\end{tabular}

Nota: EP = Erro Padrão.

No modelo 3, foram inseridas as variáveis do pilar 1 - qualidade do ensino. Nesse pilar, duas variáveis não apresentaram efeitos significativos: qualidade do material didático e práticas pedagógicas. A variável esporte tem efeito negativo sobre o desempenho. Em geral, escolas que possuem atividades extras de esporte tendem a ter um desempenho em média 1,9 pontos menor. As demais variáveis possuem efeitos positivos sobre o desempenho, com destaque para as variáveis percentual do currículo cumprido e professor corrige dever de casa, as quais confirmam a essencialidade da oportunidade e do monitoramento para a efetividade do aprendizado. Para Muijs, Kyriakides, Van der Werf, Creemers, Timperley, \& Earl (2014), a oportunidade de aprender, concebida 
frequentemente como a cobertura curricular, é um dos achados mais consistentes nos estudos relativos à efetividade do ensino conduzidos em vários países. Já a correção do dever de casa é um indicativo de que a escola monitora o progresso dos estudantes, o que também encontra respaldo na literatura na área de eficácia escolar (Reynolds et al., 2014). Com a inclusão do pilar 1 no modelo, $30 \%$ da variância do nível 2 é explicada, $15 \%$ a mais do que o modelo anterior.

Tabela 5

Modelos 3,4 e 5 da análise multinível - Prova Brasil 2009

\begin{tabular}{|c|c|c|c|c|c|c|c|}
\hline \multirow{2}{*}{$\begin{array}{c}\text { Fator/ } \\
\text { Pilar }\end{array}$} & \multirow[b]{2}{*}{ Efeito fixo } & \multicolumn{2}{|c|}{ Modelo 3} & \multicolumn{2}{|c|}{ Modelo 4} & \multicolumn{2}{|c|}{ Modelo 5} \\
\hline & & Efeito & EP & Efeito & EP & Efeito & EP \\
\hline & Intercepto & 164,6 & 1,53 & 167,7 & 1,56 & 166,8 & 1,49 \\
\hline Controle & NSE & 4,43 & 0,06 & 4,42 & 0,06 & 4,25 & 0,06 \\
\hline \multirow{3}{*}{$\begin{array}{l}\text { Suporte } \\
\text { Familiar }\end{array}$} & Ver mãe lendo & 1,14 & 0,13 & 1,13 & 0,13 & 1,09 & 0,13 \\
\hline & Número de livros & 4,30 & 0,07 & 4,30 & 0,07 & 4,33 & 0,07 \\
\hline & Suporte familiar & $-2,06$ & 0,10 & $-2,07$ & 0,10 & $-2,06$ & 0,10 \\
\hline \multirow{9}{*}{ Pilar 1} & Esporte & $-1,90$ & 0,44 & $-2,21$ & 0,43 & $-1,83$ & 0,41 \\
\hline & Artes & 1,29 & 0,32 & 0,81 & 0,31 & $-0,15$ & 0,30 \\
\hline & Escolaridade diretor & 3,29 & 0,26 & 3,19 & 0,26 & 2,05 & 0,25 \\
\hline & Adequação currículo & 2,53 & 0,47 & 2,05 & 0,46 & 2,04 & 0,44 \\
\hline & $\%$ Currículo & 7,30 & 0,24 & 6,91 & 0,24 & 5,94 & 0,23 \\
\hline & Escolaridade professor & 4,26 & 0,31 & 4,17 & 0,31 & 3,11 & 0,29 \\
\hline & Ter material didático & 2,57 & 0,17 & 2,38 & 0,17 & 1,99 & 0,16 \\
\hline & Material em tempo & 2,32 & 0,54 & 1,97 & 0,53 & 1,58 & 0,52 \\
\hline & Prof. corrige dever & 7,11 & 0,12 & 7,12 & 0,12 & 7,14 & 0,12 \\
\hline \multirow{3}{*}{ Pilar 3} & Indisciplina & & & $-1,91$ & 0,43 & $-2,86$ & 0,41 \\
\hline & Faltar aulas - aluno & & & $-2,54$ & 0,25 & $-2,80$ & 0,24 \\
\hline & Atividades de tutoria & & & 4,07 & 0,35 & 2,28 & 0,34 \\
\hline \multirow[t]{7}{*}{ Pilar 4} & Equipamentos & & & & & 5,62 & 0,15 \\
\hline & Efeito aleatório & & & & & & \\
\hline & Nível 1 - aluno & \multicolumn{2}{|c|}{$1.674,9$} & \multicolumn{2}{|c|}{$1.674,9$} & \multicolumn{2}{|c|}{$1.674,7$} \\
\hline & Nível 2 - escola & \multicolumn{2}{|c|}{259,6} & \multicolumn{2}{|c|}{254.1} & \multicolumn{2}{|c|}{229,2} \\
\hline & \multicolumn{7}{|l|}{ Variância Explicada } \\
\hline & Nível 1 - aluno & \multicolumn{2}{|c|}{$1,9 \%$} & \multicolumn{2}{|c|}{$1,9 \%$} & \multicolumn{2}{|c|}{$1,9 \%$} \\
\hline & Nível 2 - escola & \multicolumn{2}{|c|}{$30,0 \%$} & \multicolumn{2}{|c|}{$31,5 \%$} & \multicolumn{2}{|c|}{$38,2 \%$} \\
\hline
\end{tabular}

Nota: EP = Erro Padrão.

Em seguida, acrescentou-se a única variável do pilar 2, teste de seleção. Essa variável não apresentou efeito significativo. Dessa forma, o pilar 2 foi retirado do modelo.

No pilar 3, somente uma variável não apresentou efeito significativo, professores faltar às aulas. Dentre as outras variáveis, duas apresentaram efeitos negativos - 
indisciplina e alunos faltar às aulas - e uma apresentou efeito positivo - atividades de tutoria. Esses resultados são bem coerentes com o esperado. Quanto mais há indisciplina na sala e quanto mais os alunos faltam às aulas, menor é o tempo efetivo de aprendizado e isso tende a impactar negativamente o desempenho. O pilar tempo de aprendizado está bastante relacionado com o ambiente de sala de aula e com a capacidade do professor de proporcionar um ambiente ordeiro e de interação, bem como de saber lidar com a competição e colaboração (Creemers \& Kyriakides, 2008). Por outro lado, quando a escola oferece atividades de tutoria, ela está aumentando o tempo de aprendizado dos alunos. A inclusão do pilar 3 aumentou 1,5\% da variância explicado do nível 2.

Quando as variáveis do pilar 4 foram inseridas no modelo, somente uma variável apresentou efeito significativo: equipamentos. Aumentar um ponto na escala de equipamentos eleva em média 5,62 pontos na escala de proficiência. Ou seja, escolas mais bem equipadas contribuem para um melhor aprendizado, corroborando as conclusões de Murillo e Roman (2011). O pilar 4 - recursos escolares - apesar de possuir somente uma variável como representante, contribuiu com um aumento de 6,7\% da variância explicada do nível 2.

No modelo 6, verificou-se o efeito randômico dos coeficientes de inclinação das variáveis do nível do aluno (Tabela 6). Nesse passo, procurou-se verificar se as variáveis do nível 1 se comportam diferentemente entre as escolas. Três variáveis apresentaram efeito randômico: NSE, número de livros e professor corrigir dever de casa. Isso significa que o efeito dessas variáveis varia consideravelmente entre as escolas.

No modelo 7, efeitos de interação entre níveis foram verificados. A interação foi testada entre o NSE do aluno e as variáveis escolares do nível 2, o que nos permite uma análise de equidade. Três efeitos entre níveis foram identificados. O primeiro foi entre NSE e alunos faltar às aulas. Nota-se um efeito negativo que indica que, em escolas em que há 
problemas de alunos faltando às aulas, o efeito do nível socioeconômico sobre o desempenho é menor. Uma das interpretações possíveis para esse resultado é que a escola vem a somar-se à potencialidade do nível socioeconômico do aluno em contribuir com um melhor desempenho acadêmico. Logo, quando os alunos faltam muito às aulas, o apoio da escola é menor, o que diminui o efeito do nível socioeconômico. Todavia, esse resultado deve ser interpretado com parcimônia e merece mais investigações.

Os outros dois efeitos possuem sinais positivos, ou seja, em escolas em que mais professores afirmam ter cumprido um percentual maior do currículo e em que o material didático chega em tempo hábil para o ano letivo, maior o impacto do nível socioeconômico sobre o desempenho. De modo geral, esses resultados convergem para os encontrados por outros pesquisadores (Albanez, Ferreira, \& Franco, 2002; Soares \& Alves, 2003; Soares, 2004), os quais apontam que fatores escolares que contribuem para uma melhora no desempenho tornam a escola mais desigual, aumentando a importância do nível socioeconômico do aluno para o seu desempenho. 
Tabela 6

Modelos 6 e 7 da análise multinível - Prova Brasil 2009

\begin{tabular}{|c|c|c|c|c|c|}
\hline \multirow{2}{*}{$\begin{array}{l}\text { Fator/ } \\
\text { Pilar }\end{array}$} & \multirow[b]{2}{*}{ Efeito fixo } & \multicolumn{2}{|c|}{ Modelo 6} & \multicolumn{2}{|c|}{ Modelo 7} \\
\hline & & Efeito & $\mathrm{EP}$ & Efeito & EP \\
\hline & Intercepto & 168,3 & 1,45 & 170,9 & 1,51 \\
\hline Controle & NSE & 4,21 & 0,06 & 2,60 & 0,27 \\
\hline \multirow{3}{*}{$\begin{array}{l}\text { Suporte } \\
\text { Familiar }\end{array}$} & Ver mãe lendo & 1,09 & 0,13 & 1,10 & 0,13 \\
\hline & Número de livros & 4,28 & 0,07 & 4,28 & 0,07 \\
\hline & Suporte familiar & $-2,10$ & 0,10 & $-2,10$ & 0,10 \\
\hline \multirow{9}{*}{ Pilar 1} & Esporte & $-1,94$ & 0,40 & $-1,93$ & 0,40 \\
\hline & Artes & $-0,09$ & 0,29 & $-0,09$ & 0,29 \\
\hline & Escolaridade diretor & 2,04 & 0,24 & 2,05 & 0,24 \\
\hline & Adequação currículo & 1,88 & 0,43 & 1,87 & 0,43 \\
\hline & \% Currículo & 5,58 & 0,23 & 4,85 & 0,29 \\
\hline & Escolaridade professor & 3,21 & 0,28 & 3,23 & 0,28 \\
\hline & Ter material didático & 1,89 & 0,16 & 1,89 & 0,16 \\
\hline & Material em tempo & 1,31 & 0,51 & $-0,72$ & 0,60 \\
\hline & Prof. corrige dever & 7,42 & 0,12 & 7,41 & 0,12 \\
\hline \multirow{3}{*}{ Pilar 3} & Indisciplina & $-2,81$ & 0,40 & $-2,79$ & 0,40 \\
\hline & Faltar aulas - aluno & $-2,60$ & 0,24 & $-1,96$ & 0,31 \\
\hline & Atividades de tutoria & 2,05 & 0,33 & 2,06 & 0,33 \\
\hline \multirow[t]{11}{*}{ Pilar 4} & Equipamentos & 5,35 & 0,14 & 5,33 & 0,14 \\
\hline & Efeito aleatório & & & & \\
\hline & Nível 1 - aluno & \multicolumn{2}{|c|}{$1.659,2$} & \multicolumn{2}{|c|}{$1.659,6$} \\
\hline & Nível 2 - escola & \multicolumn{2}{|c|}{262,3} & \multicolumn{2}{|c|}{258,0} \\
\hline & NSE & \multicolumn{2}{|c|}{7,8} & \multicolumn{2}{|c|}{7,2} \\
\hline & Número de livros & \multicolumn{2}{|c|}{11,2} & \multicolumn{2}{|c|}{10,7} \\
\hline & Prof. corrige dever & \multicolumn{2}{|c|}{26,7} & \multicolumn{2}{|c|}{25,6} \\
\hline & \multicolumn{5}{|l|}{ Efeitos de Interação } \\
\hline & NSE x Faltar aulas - aluno & & & \multicolumn{2}{|c|}{$-0,36$} \\
\hline & NSE x \% Currículo & & & \multicolumn{2}{|c|}{0,42} \\
\hline & NSE x Material em tempo & & & \multicolumn{2}{|c|}{1,21} \\
\hline
\end{tabular}

Nota: $\mathrm{EP}=$ Erro Padrão.

Uma vez identificados os efeitos dos pilares educacionais sobre o desempenho acadêmico, buscou-se compreender essa relação ao longo do tempo. Foram utilizadas somente as variáveis com efeitos significativos no modelo final ${ }^{2}$. Na análise longitudinal, o nível 1 refere-se às variáveis da escola com medidas repetidas e o nível 2 contempla as variáveis fixas da escola. Primeiramente, foi realizada a análise do modelo nulo (Tabela 7). Pela correlação intraclasse, verificou-se que $28 \%$ da variabilidade do desempenho em

\footnotetext{
${ }^{2}$ Todas as variáveis com efeito positivo na análise transversal foram testadas no modelo longitudinal, mas somente foram mantidas aquelas que apresentaram efeito significativo.
} 
matemática se deve a mudanças ao longo do tempo (medidas repetidas) e que $72 \%$ da variabilidade são explicados pelas diferenças entre as escolas.

No modelo 1, foi adicionada a variável ocasião (tempo) que representa a taxa média de aumento da proficiência ao longo dos anos. Nesse modelo, o intercepto $(241,6)$ representa a proficiência média em matemática no primeiro ano de avaliação (2007). O efeito da variável ocasião significa que, em média, a proficiência em matemática nas escolas aumentou 2,35 pontos a cada avaliação da Prova Brasil. Portanto, entre 2007 e 2013, a proficiência teve um aumento em torno de 7 pontos, um crescimento bastante tênue, se considerarmos o desvio-padrão da escala, que é 50. Com a inclusão da variável ocasião, houve uma redução da variância do primeiro nível e um pequeno aumento da variação do segundo nível. Segundo Hox (2010), esse resultado é esperado uma vez que o modelo nulo da análise longitudinal de medidas repetidas subestima a variação entre os indivíduos (escolas) e superestima a variação entre as ocasiões.

Tabela 7

Modelos 0,1 e 2 da análise multinível - Prova Brasil 2007, 2009, 2011 e 2013

\begin{tabular}{|c|c|c|c|c|c|c|c|}
\hline \multirow{2}{*}{$\begin{array}{l}\text { Fator/ } \\
\text { Pilar }\end{array}$} & \multirow[b]{2}{*}{ Efeito fixo } & \multicolumn{2}{|c|}{ Modelo 0} & \multicolumn{2}{|c|}{ Modelo 1} & \multicolumn{2}{|c|}{ Modelo 2} \\
\hline & & Efeito & EP & Efeito & $\mathrm{EP}$ & Efeito & $\mathrm{EP}$ \\
\hline & Intercepto & 245,2 & 0,23 & 241,6 & 0,25 & 206,3 & 0,73 \\
\hline & Ocasião & & & 2,35 & 0,07 & 1,97 & 0,07 \\
\hline & NSE - Variação & & & & & 3,65 & 0,25 \\
\hline & NSE - Geral & & & & & 13,75 & 0,40 \\
\hline & \multicolumn{7}{|l|}{ Efeito aleatório } \\
\hline & Nível 1 - ocasião & \multicolumn{2}{|c|}{109,3} & \multicolumn{2}{|c|}{100,0} & \multicolumn{2}{|c|}{99,5} \\
\hline & Nível 2 - escola & \multicolumn{2}{|c|}{283,4} & \multicolumn{2}{|c|}{285,7} & \multicolumn{2}{|c|}{193,2} \\
\hline & \multicolumn{7}{|l|}{ Variância Explicada } \\
\hline & \multicolumn{3}{|l|}{ Nível 1 - ocasião } & \multicolumn{2}{|c|}{$8,5 \%$} & \multicolumn{2}{|c|}{$8,9 \%$} \\
\hline & \multicolumn{3}{|l|}{ Nível 2 - escola } & \multicolumn{2}{|c|}{$-0,8 \%$} & \multicolumn{2}{|c|}{$31,8 \%$} \\
\hline
\end{tabular}

Nota: $\mathrm{EP}$ = Erro Padrão.

No modelo 2, acrescentaram-se as variáveis referentes ao nível socioeconômico. Dois efeitos foram estimados, o nível socioeconômico médio dos alunos de cada escola a cada edição da Prova Brasil (NSE - Variação) e o nível socioeconômico geral 
considerando todo o período analisado (NSE - Geral). O NSE - Variação teve um efeito positivo sobre o desempenho, indicando que, no decorrer dos anos, a cada aumento de um nível na escala socioeconômica, houve aumento de, em média, 3,65 pontos na média da escola. Esse aumento é substancial, no entanto, deve-se considerar que o esforço para se aumentar em um nível a média do nível socioeconômico da escola é bastante razoável. Já o NSE - Geral, indica o efeito estável do nível socioeconômico ou o efeito de composição (13,75 pontos). A inclusão das variáveis de NSE no modelo explica mais de $30 \%$ da variância do nível 2. Demonstra, portanto, que as variáveis de NSE têm uma maior contribuição na explicação das diferenças entre escolas e que a variação do nível socioeconômico dos alunos recebidos pelas escolas ao longo dos anos foi pequena.

No modelo 3, foram incluídas as variáveis de medidas repetidas da escola, baseadas nos pilares do modelo de prosperidade. A entrada dessas variáveis no modelo aumenta o percentual de variância explicada para 12,2\% no nível 1 e 43,1\% no nível 2.

Como esperado, a única variável com efeito negativo foi indisciplina, pois ela tende a diminuir o tempo efetivo de aprendizado. As demais variáveis possuem efeito positivo sobre o desempenho, com destaque para o percentual do currículo cumprido, material didático em tempo hábil e professor corrigir dever de casa, as quais possuem efeitos de maior magnitude. Esses resultados seguem a mesma tendência da análise transversal. Destaca-se que, mesmo após a inclusão de todas as variáveis referentes às ações escolares, o percentual de variância explicada do nível 1 é razoavelmente pequeno. Isso indica que não houve variações grandes desses pilares ao longo dos anos, estes estando de certo modo estagnados. Indica também que outros fatores escolares variaram ao longo dos anos, os quais não foram captados neste estudo. 
Tabela 8

Modelo 3 e modelo final da análise multinível - Prova Brasil 2007, 2009, 2011 e 2013

\begin{tabular}{|c|c|c|c|c|c|c|c|}
\hline \multirow{2}{*}{$\begin{array}{l}\text { Fator/ } \\
\text { Pilar }\end{array}$} & \multirow[b]{2}{*}{ Efeito fixo } & \multicolumn{2}{|c|}{ Modelo 3} & \multicolumn{2}{|c|}{$\begin{array}{c}\text { Modelo Final } \\
\text { Rede Municipal }\end{array}$} & \multicolumn{2}{|c|}{$\begin{array}{l}\text { Modelo Final } \\
\text { Rede Estadual }\end{array}$} \\
\hline & & Efeito & EP & Efeito & EP & Efeito & EP \\
\hline & Intercepto & 192,2 & 0,97 & 194,4 & 1,04 & 192,0 & 0,94 \\
\hline & Ocasião & 1,89 & 0,08 & 2,09 & 0,08 & 2,01 & 0,09 \\
\hline & NSE - Variação & 3,39 & 0,25 & 3,57 & 0,24 & 3,79 & 0,24 \\
\hline & NSE - Geral & 13,26 & 0,38 & 12,17 & 0,39 & 13,29 & 0,36 \\
\hline \multirow{4}{*}{ Pilar 1} & Adequação currículo & 1,07 & 0,25 & 1,02 & 0,25 & 0,87 & 0,24 \\
\hline & $\%$ Currículo & 2,09 & 0,13 & 1,95 & 0,13 & 1,91 & 0,13 \\
\hline & Material em tempo & 2,35 & 0,26 & 2,34 & 0,26 & 2,17 & 0,26 \\
\hline & Prof. corrige dever & 3,25 & 0,11 & 3,18 & 0,11 & 3,09 & 0,11 \\
\hline \multirow{2}{*}{ Pilar 3} & Indisciplina & $-1,69$ & 0,23 & $-1,53$ & 0,22 & $-1,35$ & 0,22 \\
\hline & Atividades de tutoria & 1,18 & 0,18 & 1,45 & 0,18 & 1,43 & 0,18 \\
\hline \multirow[t]{27}{*}{ Pilar 4} & Infraestrutura & 1,24 & 0,16 & 1,12 & 0,16 & 1,20 & 0,16 \\
\hline & $\mathrm{AC}$ & & & $-11,30$ & 3,29 & & \\
\hline & AM & & & $-16,40$ & 1,65 & & \\
\hline & PA & & & $-5,45$ & 1,42 & & \\
\hline & AP & & & & & $-20,97$ & 2,05 \\
\hline & RR & & & & & $-10,33$ & 1,07 \\
\hline & TO & & & & & $-5,93$ & 1,61 \\
\hline & MA & & & $-4,62^{-}$ & 1,20 & $-5,17^{-}$ & 1,48 \\
\hline & RN & & & & & $-3,69^{-}$ & 1,42 \\
\hline & PB & & & $-4,81$ & 1,22 & $-9,95$ & 1,63 \\
\hline & PE & & & & & $-14,09^{+}$ & 0,79 \\
\hline & SE & & & & & $-9,29$ & 1,69 \\
\hline & BA & & & $-3,17^{-}$ & 0,92 & $-8,59^{-}$ & 0,97 \\
\hline & MG & & & 12,42 & 0,97 & 13,93 & 0,57 \\
\hline & RJ & & & & & $-7,54$ & 0,98 \\
\hline & SP & & & & & $-2,23$ & 0,38 \\
\hline & MS & & & $10,32^{-}$ & 1,45 & 4,09 & 0,87 \\
\hline & MT & & & & & $-2,82^{-}$ & 1,13 \\
\hline & \multicolumn{7}{|l|}{ Efeito aleatório } \\
\hline & Nível 1 - ocasião & 95,9 & & 75,7 & & 76,2 & \\
\hline & Nível 2 - escola & 161,3 & & 134,3 & & 107,0 & \\
\hline & Ocasião & & & 8,3 & & 8,1 & \\
\hline & Atividades de tutoria & & & 17,8 & & 15,8 & \\
\hline & Prof. corrige dever & & & 7,7 & & 7,3 & \\
\hline & \multicolumn{7}{|l|}{ Variância Explicada } \\
\hline & Nível 1 - ocasião & \multicolumn{2}{|c|}{$12,2 \%$} & & & & \\
\hline & Nível 2 - escola & \multicolumn{2}{|c|}{$43,1 \%$} & & & & \\
\hline
\end{tabular}

Nota: $\mathrm{EP}=$ Erro Padrão.

Posteriormente, foram incluídas no modelo as variáveis escolares fixas ao longo do tempo. Nesse caso, utilizou-se a relação de UF e dependência administrativa de cada escola. Como são muitas combinações possíveis, as análises foram realizadas em dois momentos. Uma primeira análise considerando a rede municipal e depois a rede estadual. A rede federal não foi contemplada por se tratar de poucas escolas. A Tabela 8 apresenta 
as UF/redes com efeitos significativos. Ressalta-se o efeito positivo sobre o desempenho da rede municipal e estadual de Minas Gerais e da rede municipal e estadual de Mato Grosso do Sul. Os estudantes que frequentam escolas dessas redes têm em geral desempenho superior aos demais. Por outro lado, o desempenho dos estudantes em outras UF/redes é significativamente abaixo da média, como é o caso dos estudantes da rede municipal do Amazonas e da rede estadual do Amapá, mesmo sendo feito controle pelo nível socioeconômico. Esse resultado é bastante interessante, pois demonstra o papel que a UF/rede pode exercer na melhoria da qualidade do sistema. Contribui ainda para deixar mais transparentes aquelas UF/redes que têm trabalhado bem ao longo dos anos.

Ao se analisar o componente aleatório das variáveis do nível 1, identificou-se que ocasião, atividades de tutoria e professor corrigir dever de casa possuem coeficientes de inclinação que variam entre as escolas. Portanto, há grande variabilidade entre as escolas quanto à melhora no desempenho em matemática ao longo dos anos. Além disso, nota-se que atividades de tutoria e professor corrigir dever de casa são variáveis bastante dependentes do gerenciamento da escola, o que pode explicar a maior variabilidade.

Por fim, ainda na Tabela 8, são apresentados os resultados da análise de interação entre níveis. A única relação testada foi a interação de ocasião com UF/rede. Os sinais de e + sobrescritos indicam as UF/redes com efeitos de interação significativos e a direção desse efeito. Observa-se, por exemplo, que na rede estadual de Pernambuco, apesar de os estudantes terem, em média, desempenhos mais baixos, as escolas tiveram uma taxa de aumento da proficiência em matemática maior. Esses resultados são interessantes porque apontam novamente o quanto as $\mathrm{UF} / \mathrm{redes}$ podem ajudar as escolas a melhorar seus desempenhos, mesmo estando em níveis distintos de proficiência. 


\section{Considerações Finais}

A partir da análise transversal foi possível verificar o efeito dos pilares educacionais sobre o desempenho em matemática. De modo geral, este estudo confirma a importância dos pilares educacionais propostos pelo modelo de prosperidade educacional. Com a inclusão dos pilares 1, 3 e 4 no modelo, pelo menos 35,8\% da variância do nível 2 foi explicada.

Duas conclusões podem ser extraídas desses resultados. A primeira é que se ratificam os pilares processuais qualidade do ensino e tempo de aprendizado como fundamentais para compreensão dos motivos de umas escolas serem mais eficazes do que outras, bem como mostram que o pilar recursos escolares ainda faz bastante diferença no cenário brasileiro. A segunda conclusão é a de que esses pilares são muito promissores e podem explicar ainda mais as diferenças entre as escolas, uma vez que as medidas utilizadas podem ser aperfeiçoadas. Há autores inclusive que advertem para o fato de que o papel da escola e da turma pode ser subestimado, se medidas apropriadas não forem utilizadas. Por exemplo, tem-se a hipótese de que as melhores medidas de qualidade de ensino são obtidas por meio de observação direta (Creemers \& Reezing, 1996; Reynolds \& Teddlie, 2000).

Infelizmente, não foi possível verificar a relação entre o pilar 2 e o desempenho acadêmico, mas esse resultado era esperado dado que a medida utilizada não era uma boa representante do pilar (Karino, 2016a). Com isso, novamente aponta-se a necessidade de se investir no desenvolvimento de melhores medidas. O ambiente inclusivo é pilar essencial para a mensuração da qualidade de uma escola. A valorização da diversidade é a base para a promoção de uma educação para todos.

Se por um lado o desenvolvimento de medidas ainda é necessário, por outro, este estudo já avança ao fazer uso de medidas interpretáveis. Ter a indicação do que significa estar no nível 1 de uma escala de equipamentos ou de infraestrutura, por exemplo, dá 
concretude aos resultados e aproxima a pesquisa das ações. Outra contribuição é a realização de uma pesquisa de análise de fatores associados a partir de uma teoria clara a ser validada e que pode direcionar pesquisas futuras. Evidenciou-se na pesquisa transversal o potencial da escola no aprendizado dos alunos. Contudo, permanece ainda o desafio de descobrir como a escola pode diminuir diferenças anteriores e, consequentemente, diminuir desigualdades.

Ao se utilizar uma análise longitudinal de cortes transversais, objetivava-se compreender melhor o aumento no desempenho dos estudantes nos últimos anos, distinguindo melhor as mudanças de desempenho decorrentes de mudanças contextuais, daquelas provenientes de fatores intrinsecamente escolares. Verificou-se que entre 2007 e 2011 o desempenho em matemático aumentou em média 2,35 pontos a cada avaliação. A maior parte da variabilidade no desempenho ao longo dos anos é explicada pela variância entre escolas $(72 \%)$ e não por mudanças entre aplicações (28\%). As medidas de nível socioeconômico, por exemplo, pouco contribuíram para a explicação das mudanças ao longo das aplicações, mas é responsável por mais de 30\% da variância entre escolas. Já os pilares educacionais explicam cerca de $3 \%$ da variância entre aplicações e aproximadamente $10 \%$ da variância entre escolas. Dessa forma, nota-se que tanto o contexto social, quanto os pilares educacionais apresentaram um efeito estável mais relevante do que um efeito variável ao longo dos anos. Por fim, chama a atenção também na análise longitudinal o papel que as UF/redes podem exercer no sistema educacional.

Uma limitação dessa pesquisa como um todo, refere-se à perda amostral, sobretudo na análise longitudinal. Uma análise exploratória da média em matemática mostra que o grupo selecionado para o estudo longitudinal (escolas presentes nas quatro avaliações 2007, 2009, 20011 e 2013) apresenta desempenho superior aos demais grupos (com uma, duas ou três participações nas avaliações). Todavia, o tratamento dos casos missing 
tornaria a pesquisa muito complexa, pois as razões que justificam a ausência da escola na Prova Brasil são bastante abrangentes e difíceis de serem mapeadas: poucos alunos matriculados naquele ano, falta de verbas para manutenção da escola, dificuldades em responder ao censo escolar (condição para participar da avaliação). Torna-se claro, portanto, que os resultados aqui apresentados referem-se às escolas com maior estabilidade, presentes desde 2007 no sistema educacional brasileiro, o que por si só já contribui para um melhor desempenho dos estudantes. Recomenda-se, a partir dessa investigação, a realização de estudos futuros que busquem compreender os motivos que levam as escolas a não participarem da Prova Brasil ao longo dos anos.

Para concluir, a premissa dos estudos que avaliam eficácia escolar é a de que as escolas podem melhorar as condições dos estudantes e compensar diferenças que resultam de seu contexto social (Carvallo-Pontón, 2010). Nos países em desenvolvimento, acreditar nessa premissa é ainda mais importante. Assim sendo, num sentido mais amplo e social, espera-se que os resultados desta pesquisa possam contribuir para a ampliação de discussões que, por ventura, poderão culminar em políticas públicas que permitirão a melhoria da qualidade da educação ofertada neste país.

\section{Referências}

Albanez, A., Ferreira, F., \& Franco, F. (2002) Qualidade e equidade no ensino fundamental brasileiro. Pesquisa e Planejamento Econômico, 32(3), 453-475.

Andrade, J. M., \& Laros, J. A. (2007). Fatores associados ao desempenho escolar: Um estudo multinível com os dados do SAEB/2001. Psicologia: Teoria e Pesquisa, 23, $33-42$.

Bickel, R. (2007). Multilevel analysis for applied research: It's just regression! New York: The Guilford Press.

Brasil (2005). Portaria n. 931, de 21 de março de 2005. Ministério da Educação: Autor.

Carvallo-Pontón, M. (2010). Eficacia escolar: antecedentes, hallazgos y futuro. Revista Internacional de Investigación en Educación, 3, 199-214. 
Coleman, J. S., Campbell, E., Hobson, C., McPartland, J., Mood, A., Weinfeld, R., \& York, R. (1966). Equality of educational opportunity. Washington: US Department of Health, Education \& Welfare.

Creemers, B. P. M., \& Kyriakides, L. (2008). The dynamics of educational effectiveness: A contribution to policy, practice and theory in contemporary schools. London: Routledge.

Creemers, B. P. M., \& Reezing, G. J. (1996). School level conditions affecting the effectiveness of institution. School Effectiveness and School Improvement, 7(3), 197228.

DiPrete, T. A., \& Grusky, D. B. (1990). The multilevel analysis of trends with repeated cross-sectional data. In C. C. Clogg (Ed.), Sociological methodology. London: Blackwell.

Ferrão, M. E. (2003). Introdução aos modelos de regressão multinível em educação. Campinas: Editora Komedi.

Ferrão, M. E., \& Couto, A. (2013). Indicador de valor acrescentado e tópicos sobre a consistência e estabilidade: Uma aplicação ao Brasil. Ensaio: Avaliação de Políticas Públicas Educacionais, 21(78), 131-164.

Fletcher, P. R. (1998). À procura do ensino eficaz. Relatório técnico. Brasília: MECDAEB.

Forquin, J. C. (1995). A sociologia das desigualdades de acesso à educação: Principais orientações, principais resultados desde 1965. In J. C. Forquin (Ed.), Sociologia da educação: dez anos de pesquisas. Petrópolis: Vozes.

Franco, C., \& Bonamino, A. (2005). A pesquisa sobre característica de escolas eficazes no Brasil: Breve revisão dos principais achados e alguns problemas em aberto. Educação on-line: Revista do Programa de Pós-graduação em Educação, 1, 1-13.

Franco, C., Brooke, N., \& Alves, F. (2008). Estudo longitudinal sobre qualidade e equidade no ensino fundamental brasileiro: GERES 2005. Ensaio: Avaliação de Políticas Públicas Educacionais, 16(61), 625-638.

Franco, C., Ortigão, I., Albernaz, A., Bonamino, A., Aguiar, G., Alves, F., \& Sátyro, N. (2007). Qualidade e equidade em educação: reconsiderando o significado de "fatores intraescolares". Ensaio: Avaliação de Políticas Públicas Educacionais, 15(55), $277-$ 298.

Hox, J. (2010). Multilevel analysis: Techniques and applications. Mahwah, New Jersey: Lawrence Erlbaum Associates.

Jesus, G. R., \& Laros, J. A. (2004). Eficácia escolar: Regressão multinível com dados de avaliação em larga escala. Avaliação Psicológica, 3(2), 93-106. 
Karino, C. A. (2016a). Pillars of good education: Analysing the Brazilian school system. Manuscrito em preparação.

Karino, C. A. (2016b). Avaliação da igualdade e da equidade do Sistema educacional público brasileiro. Manuscrito em preparação.

Karino, C. A., Vinha, L. G. A., \& Laros, J. A. (2014). Os questionários do SAEB: O que eles realmente medem? Estudos em Avaliação Educacional, 25(59), 270-297.

Kreft, I. G. G., \& De Leeuw, J. (1998). Introducing multilevel modeling. London: Sage Publications.

Laros, J. A., Marciano, J. L. P., \& Andrade, J. M. (2010). Fatores que afetam o desempenho na prova de matemática do SAEB: Um estudo multinível. Avaliação Psicológica, 9(2), 173-186.

Lee, V. L. (2008). Utilização de modelos lineares hierárquicos lineares para estudar contextos sociais: O caso dos efeitos da escola. In N. Brooke e J. F. Soares (Eds.), Pesquisa em eficácia escolar: origem e trajetórias (pp. 273-296). Belo Horizonte: Editora UFMG.

Muijs, D., Kyriakides, L., Van der Werf, G., Creemers, B., Timperley, H., \& Earl, L. (2014). State of the art - teacher effectiveness and professional learning. School Effectiveness and School improvement: An International Journal of Research, Policy and Practice, 25(2), 231-256.

Murillo, F. J., \& Roman, M. (2011). School infrastructure and resources do matter: analysis of the incidence of school resources on the performance of Latin American students. School Effectiveness and School Improvement, 22(1), 29-50.

Raudenbush, S. W., \& Chan, W. (1993). Application of a hierarchical linear model to the study of adolescent deviance in an overlapping cohort design. Journal of Consulting and Clinical Psychology, 61(6), 941-951.

Raudenbush, S. W., Bryk, A. S., Cheong, Y. F., Congdon, R. T., \& du Toit, M. (2011). HLM 7: Hierarchical linear and nonlinear modeling. Lincolnwood, IL: Scientific Software International.

Reynolds, D., \& Teddlie, C. (2000). The process of school effectiveness. In C. Teddlie, \& D. Reynolds (Eds.), The international handbook of school effectiveness research (pp. 134-159). New York: Routledge.

Reynolds, D., Sammons, P., De Fraine, B., Van Damme, J., Townsend, T., Teddlie, C., \& Stringfield, S. (2014). Educational effectiveness research (EER): A state-of-the-art review. School Effectiveness and School Improvement: An International Journal of Research, Policy and Practice, 25(2), 197-230. 
Sammons, P., Hillman, J., \& Mortimore, P. (1995). Key characteristics of effective schools: A review of school effectiveness research. London: Office for Standards in Education [OFSTED].

Soares Neto, J. J., Jesus, G. R., Karino, C. A., \& Andrade, D. F. (2013). Uma escala para medir a infraestrutura escolar. Estudos em Avaliação Educacional, 24(54), 78-99.

Soares, J. F. (2004). Qualidade e equidade na educação brasileira: A evidência do SAEB2001. Archivos Analíticos de Políticas Educativas, 12(38), 1-28.

Soares, J. F., \& Alves, M. T. G. (2003). Desigualdades raciais no sistema brasileiro de educação básica. Educação e Pesquisa, 29(1), 147-165.

Soares, J. F., Alves, M. T. G., \& Oliveira, R. M. (2001). O efeito de 248 escolas de nível médio no vestibular da UFMG nos anos de 1998, 1999 e 2000. Estudos em Avaliação Educacional, 24, 69-117.

Teddlie, C., Stringfield, S., \& Reynolds, D. (2000). Context issues within school effectiveness research. In C. Teddlie, \& D. Reynolds (Eds.), The international handbook of school effectiveness research (pp. 160-185). New York: Routledge.

Timmermans, A. C., Snijders, T. A. B., \& Bosker, R. J. (2012). In search of value added in the case of complex school effects. Educational and Psychological Measurement, 73(2), 210-228.

Van Den Eeden, P., Hox, J., \& Hauer, J. (1990). Theory and model in multilevel research: Convergence or divergence? Amsterdam: SISWO.

Vinha, L. G. A. (2016). Estudos longitudinais e tratamento de dados ausentes em avaliações educacionais. Tese de Doutorado, Universidade de Brasília, Brasília.

Vinha, L. G. A., Karino, C. A., \& Laros, J. A. (2016). Factors associated with Mathematics in Brazilian education. Psico-USF, 21(1), 87-100.

White, K. R. (1982). The relation between socioeconomic status and academic achievement. Psychological Bulletin, 91(3), 461-481.

Willms, J. D. (1992). Monitoring school performance. Washington, D.C.: The Falmer Press.

Willms, J. D. (2010). School composition and contextual effects on student outcomes. Teachers College Record, 112(4), 1008-1037.

Willms, J. D. (2015). Educational prosperity. Fredericton, NB: The Learning Bar Inc.

Willms, J. D., \& Raudenbush, S. W. (1989). A longitudinal hierarchical linear model for estimating school effects and their stability. Journal of Educational Measurement, 26, 209-232. 


\section{Conclusão}

Esta tese teve como objetivo geral avaliar o sistema educacional brasileiro em termos de o quanto ele tem proporcionado igualdade, equidade e eficácia. Para tanto, no Estudo 1 foi realizada uma revisão sistemática da literatura brasileira. Com base nessa revisão ficou claro que, entre os desafios da área de eficácia escolar, está o delineamento e execução de pesquisas longitudinais, a necessidade de se encontrar respostas para a promoção de escolas mais eficazes e mais equânimes e a necessidade de se construir medidas válidas e com embasamento teórico. Também ficou evidenciada a necessidade de se investir em outras estratégias de análises que possibilitem uma melhor estimação tanto do efeito-escola quanto das variáveis que contribuem para a promoção da eficácia escolar. Essa revisão apontou necessidades na área, tendo sido fundamental para o delineamento dos demais três estudos realizados.

No Estudo 2, definiu-se a teoria que seria utilizada como base para a execução da pesquisa: o modelo de prosperidade educacional. Disseminar e aplicar esse modelo no contexto brasileiro foi um passo importante. Buscava-se o desenvolvimento de uma tese mais teoricamente dirigida, diferenciando-se das pesquisas que incluem variáveis no modelo multinível sem uma hipótese teórica sólida. Infelizmente, não foi possível construir medidas adequadas para todos os pilares do modelo.

Nos Estudos 3 e 4, realizou-se propriamente a avaliação de igualdade, equidade e eficácia. No terceiro estudo, o objetivo principal foi verificar diferenças de desempenho acadêmico e diferenças de acesso a recursos escolares conforme a condição socioeconômica do estudante. $\mathrm{O}$ intuito era o desenvolvimento de um estudo que retratasse 
as desigualdades existentes no sistema educacional brasileiro de forma simples e compreensível para pessoas fora do meio acadêmico. Por isso, optou-se por trabalhar com a chance de sucesso e a chance de acesso. Além disso, o estudo foi delineado para ter a análise de igualdade e equidade como fim e não como variáveis moderadoras como já realizado em outros estudos brasileiros.

Por fim, no quarto estudo, o desafio era a análise longitudinal com medidas repetidas das escolas. Buscou-se compreender a relação entre os pilares educacionais e a eficácia escolar e a variabilidade do desempenho e dos pilares educacionais ao longo dos anos. Os resultados confirmam a importância dos pilares definidos pelo modelo de prosperidade educacional, mas eles não foram suficientes para explicar toda a variância. Ademais, tanto no Estudo 3 quanto no 4, as análises por UF/rede administrativa permitiram identificar sistemas mais eficazes e aqueles que tem tido sucesso na promoção de igualdade e equidade.

De modo geral, destaca-se como principais contribuições acadêmicas desses quatro estudos: (1) o uso de uma nova perspectiva teórica, acreditando que esse modelo vem a contribuir para o desenvolvimento de uma área mais teoricamente dirigida e, consequentemente, para o direcionamento de avanços teóricos; (2) o fortalecimento da concepção de que os sistemas educacionais devem prover qualidade e equidade, sendo este o grande desafio atual da área; (3) a indicação da razão de chance dos alunos pobres atingirem o nível adequado de desempenho e terem acesso a recursos escolares; (4) a construção de medidas escolares com o uso da teoria de resposta ao item que permite a comparabilidade dos fatores ao longo dos anos e, sobretudo, a interpretação pedagógica dessas medidas; (5) a proposição de investigações na área de eficácia escolar com o uso de modelo multinível de medidas repetidas, que possibilita a análise longitudinal do sistema escolar; e (6) a partir dessa análise longitudinal, advieram compreensões importantes 
acerca do avanço no desempenho médio das escolas ao longo dos anos e do quanto esse avanço está relacionado a melhorias nas condições escolares.

Entende-se que a realização dos quatro estudos permitiu cumprir adequadamente o objetivo geral da tese, bem como contribuiu para um avanço da área. Reforçou-se a tese de que as escolas podem fazer a diferença e de que os pilares do modelo de prosperidade educacional explicam uma parcela significativa da variabilidade entre as escolas. Todavia, esses pilares não foram suficientes para explicar as mudanças ao longo dos anos, o que demanda a execução de novas pesquisas.

Espera-se, por fim, que os resultados desses estudos possam transcender o âmbito acadêmico, atingindo a esfera social e política. Demonstra-se aqui que o sistema educacional brasileiro enfrenta dificuldades em fazer seus alunos alcançarem os níveis adequados de aprendizagem ao final da educação básica, em prover o acesso aos recursos escolares apontados como essenciais para a prosperidade educacional, bem como a distribuição desses recursos não ocorre de forma equânime para estudantes de diferentes níveis socioeconômicos. Uma vez que a situação educacional brasileira torna-se mais evidente, espera-se contribuir para o enriquecimento do debate crítico, bem como instigar políticas educacionais. 